\title{
Enantioselective Epoxidation of Conjugated cis-Enynes by Chiral Dioxirane
}

\author{
Christopher P. Burke and Yian Shi* \\ Department of Chemistry \\ Colorado State University \\ Fort Collins, CO 80523 \\ Phone: 970-491-7424 \\ Fax: 970-491-1801 \\ Email: yian@lamar.colostate.edu
}

\section{Supporting Information}

\section{Table of Contents}

General Methods

S-2

Representative procedure for Sonogashira coupling

S-2

Preparation and characterization data of enynes and epoxides

HPLC and GC data for the determination of the ee's

NMR spectral data of selected epoxides

S-28 


\section{Synthesis and characterization of conjugated cis-enynes and epoxides.}

General Methods. All commercially available reagents were used without further purification. All glassware used for the epoxidation was carefully washed with soap and water to be free of any trace metals which catalyze the decomposition of Oxone. Column chromatography was performed with silica gel (200-400 mesh). Solvents were evaporated under reduced pressure. Melting points are uncorrected.

Representative procedure for Sonogashira coupling: $\mathrm{Pd}\left(\mathrm{PPh}_{3}\right)_{4}(0.60 \mathrm{~g}, 0.50 \mathrm{mmol})$ was added at $\mathrm{rt}$ to a solution of vinyl halide $(23.4 \mathrm{mmol})$ in benzene $(25 \mathrm{~mL})$ under Ar and was stirred for $45 \mathrm{~min}$. A solution of alkyne $(11.7 \mathrm{mmol})$ in $n-\mathrm{BuNH}_{2}(12.0 \mathrm{~mL}, 120.0 \mathrm{mmol})$ was then added followed by $\mathrm{CuI}(0.35 \mathrm{~g}, 1.91 \mathrm{mmol})$ (the reaction is exothermic, so the flask was placed in a water bath before addition of $\mathrm{CuI})$. After stirring at $\mathrm{rt}$ overnight, petroleum ether (or $\mathrm{Et}_{2} \mathrm{O}$ ) $(50 \mathrm{~mL})$ was added and the mixture was poured into sat. aq. $\mathrm{NH}_{4} \mathrm{Cl}$ and extracted with more petroleum ether. The combined organic extracts were washed with sat. aq. $\mathrm{NH}_{4} \mathrm{Cl}, \mathrm{H}_{2} \mathrm{O}$, brine, dried $\left(\mathrm{Na}_{2} \mathrm{SO}_{4}\right)$, and filtered. The residue was then concentrated and purified by column (pet. ether to $1: 1$ pet. ether- $\mathrm{Et}_{2} \mathrm{O}$ ).

Ratovelomana, V.; Linstrumelle, G. Synth. Commun. 1981, 11, 917.

\section{Table 1, entry 1}

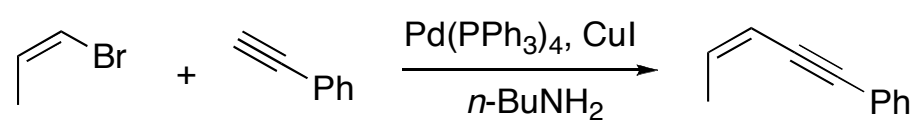

The enyne was prepared via Sonogashira coupling of cis-1-bromo-1-propene and phenylacetylene (87\%): Colorless oil; IR (NaCl): $1490 \mathrm{~cm}^{-1} ;{ }^{1} \mathrm{H}$ NMR $\left(300 \mathrm{MHz}, \mathrm{CDCl}_{3}\right) \delta$ 
7.49-7.43 (m, 2H), 7.35-7.29 (m, 3H), $6.06(\mathrm{dq}, J=10.8,6.9 . \mathrm{Hz}, 1 \mathrm{H}), 5.71(\mathrm{dq}, J=10.8,1.5$ $\mathrm{Hz}, 1 \mathrm{H}), 1.98(\mathrm{dd}, J=6.9,1.5 \mathrm{~Hz}, 3 \mathrm{H}) ;{ }^{13} \mathrm{C} \mathrm{NMR}\left(75 \mathrm{MHz}, \mathrm{CDCl}_{3}\right) \delta 138.9,131.6,128.5$, $128.2,123.9,110.2,94.1,86.4,16.3$.

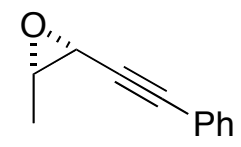

Colorless oil; $[\alpha]_{\mathrm{D}}^{25}=-40.3\left(c 0.65, \mathrm{CHCl}_{3}\right)(93 \%$ ee $) ; \mathrm{IR}(\mathrm{NaCl}): 2227,1490,1349 \mathrm{~cm}^{-1} ;{ }^{1} \mathrm{H}$ NMR (400 MHz, $\left.\mathrm{CDCl}_{3}\right) \delta$ 7.49-7.45 (m, 2H), 7.35-7.30 (m, 3H), $3.65(\mathrm{~d}, J=4.0 \mathrm{~Hz}, 1 \mathrm{H}), 3.27$ $(\mathrm{qd}, J=5.2,4.0 \mathrm{~Hz}, 1 \mathrm{H}), 1.51(\mathrm{~d}, J=5.2 \mathrm{~Hz}, 3 \mathrm{H}) ;{ }^{13} \mathrm{C} \mathrm{NMR}\left(101 \mathrm{MHz}, \mathrm{CDCl}_{3}\right) \delta 132.1,128.9$, $128.5,122.3,85.5,84.3,54.7,46.1,15.0$.

(a) Lee, N.H.; Jacobsen, E.N. Tetrahedron Lett. 1991, 32, 6533. (b) Sasaki, H.; Irie, R.; Hamada, T.; Suzuki, K.; Katsuki, T. Tetrahedron 1994, 50, 11827. (c) Tian, H.; She, X.; Shu, L.; Yu, H.; Shi, Y. J. Am. Chem. Soc. 2000, 122, 11551. (d) Tian, H.; She, X.; Yu, H.; Shu, L.; Shi, Y. J. Org. Chem. 2002, 67, 2435.

Table 1, entry 2

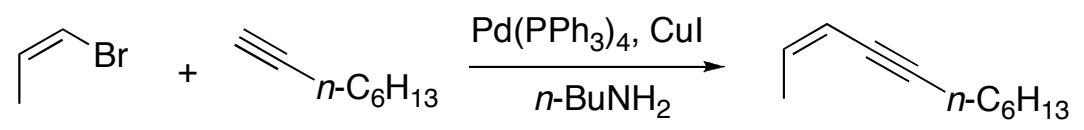

The enyne was prepared via Sonogashira coupling of cis-1-bromo-1-propene and 1-hexyne (82\%): Colorless oil; IR (NaCl): 2931, $\mathrm{cm}^{-1} ;{ }^{1} \mathrm{H}$ NMR (400 MHz, $\left.\mathrm{CDCl}_{3}\right) \delta 5.89$ (dq, $J=10.6$, $6.8 \mathrm{~Hz}, 1 \mathrm{H}), 5.50-5.45(\mathrm{~m}, 1 \mathrm{H}), 2.35(\mathrm{td}, J=7.2,2.0 \mathrm{~Hz}, 2 \mathrm{H}), 1.86(\mathrm{dd}, J=6.8,1.6 \mathrm{~Hz}, 3 \mathrm{H})$, 1.60-1.51 (m, 2H), 1.46-1.38 (m, 2H), 1.35-1.27 (m, 4H), $0.90(\mathrm{t}, J=7.2 \mathrm{~Hz}, 3 \mathrm{H}) ;{ }^{13} \mathrm{C}$ NMR $\left(101 \mathrm{MHz}, \mathrm{CDCl}_{3}\right) \delta 137.1,110.6,95.3,77.3,31.6,29.1,28.8,22.8,19.8,15.9,14.3$. 


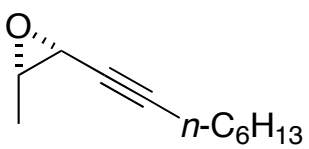

Colorless oil; $[\alpha]_{\mathrm{D}}^{25}=-34.2\left(c 0.37, \mathrm{CHCl}_{3}\right)(90 \%$ ee $)$ IR $(\mathrm{NaCl}): 2240,1456,1349 \mathrm{~cm}^{-1} ;{ }^{1} \mathrm{H}$ NMR (400 MHz, $\left.\mathrm{CDCl}_{3}\right) \delta 3.40(\mathrm{dt}, J=4.0,1.6 \mathrm{~Hz}, 1 \mathrm{H}), 3.11(\mathrm{qd}, J=5.2,4.0 \mathrm{~Hz}, 1 \mathrm{H}), 2.20$ (td, $J=7.2,1.6 \mathrm{~Hz}, 2 \mathrm{H}), 1.53-1.47(\mathrm{~m}, 2 \mathrm{H}), 1.42-1.22(\mathrm{~m}, 8 \mathrm{H}), 1.40(\mathrm{~d}, J=5.2 \mathrm{~Hz}, 3 \mathrm{H}), 0.88(\mathrm{t}, J=$ $7.2 \mathrm{~Hz}, 3 \mathrm{H}) ;{ }^{13} \mathrm{C} \mathrm{NMR}\left(101 \mathrm{MHz}, \mathrm{CDCl}_{3}\right) \delta 86.8,75.1,54.1,46.0,31.5,28.64,28.60,22.7$, $19.0,14.8,14.2$.

(a) Lee, N.H.; Jacobsen, E.N. Tetrahedron Lett. 1991, 32, 6533. (b) Tian, H.; She, X.; Shu, L.; Yu, H.; Shi, Y. J. Am. Chem. Soc. 2000, 122, 11551. (c) Tian, H.; She, X.; Yu, H.; Shu, L.; Shi, Y. J. Org. Chem. 2002, 67, 2435.

Table 1, entry 3

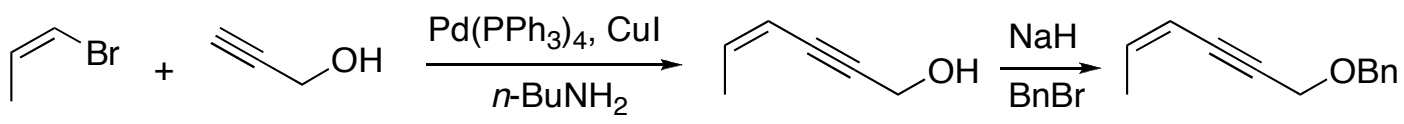

The enyne alcohol was prepared via Sonogashira coupling of cis-1-bromo-1-propene and propargyl alcohol (56\%): Yellow oil; IR (NaCl): 3334, 2199, 1363, $1011 \mathrm{~cm}^{-1} ;{ }^{1} \mathrm{H}$ NMR (400 $\left.\mathrm{MHz}, \mathrm{CDCl}_{3}\right) \delta 6.01(\mathrm{dq}, J=10.8,6.8 \mathrm{~Hz}, 1 \mathrm{H}), 5.54-5.48(\mathrm{~m}, 1 \mathrm{H}), 4.44(\mathrm{~d}, J=2.4 \mathrm{~Hz}, 2 \mathrm{H}), 1.88$ $(\mathrm{dd}, J=6.8,1.6 \mathrm{~Hz}, 3 \mathrm{H}) ;{ }^{13} \mathrm{C} \mathrm{NMR}\left(101 \mathrm{MHz}, \mathrm{CDCl}_{3}\right) \delta 139.5,109.5,91.9,82.6,51.9,16.2$.

Sodium hydride $(0.45 \mathrm{~g}$ of a $60 \%$ dispersion in mineral oil, $11.2 \mathrm{mmol})$ was placed in a flask and the mineral oil was removed by washing with pet. ether. THF $(16 \mathrm{~mL})$ was then added and the mixture was dropped to $0{ }^{\circ} \mathrm{C}$. cis-4-Hexen-2-yn-1-ol (0.98 g, $\left.10.2 \mathrm{mmol}\right)$ was then added with stirring and the mixture was warmed to rt over about $0.5 \mathrm{~h}$. Benzylbromide $(1.75 \mathrm{~g}, 10.3 \mathrm{mmol})$ was added and the mixture was allowed to stir overnight. The reaction was then quenched by addition of water and the mixture was extracted with $\mathrm{Et}_{2} \mathrm{O}$. The combined organic phases were washed with brine, dried $\left(\mathrm{Na}_{2} \mathrm{SO}_{4}\right)$, filtered, and concentrated. The residue was purified by 
column (pet. ether) to yield the benzyl ether as a colorless oil $(1.10 \mathrm{~g}, 58 \%)$; IR (NaCl): 1355 , $1088 \mathrm{~cm}^{-1} ;{ }^{1} \mathrm{H}$ NMR $\left(400 \mathrm{MHz}, \mathrm{CDCl}_{3}\right) \delta 7.41-7.28(\mathrm{~m}, 5 \mathrm{H}), 6.03(\mathrm{dq}, J=10.8,6.8 \mathrm{~Hz}, 1 \mathrm{H})$, $5.58-5.53(\mathrm{~m}, 1 \mathrm{H}), 4.65(\mathrm{~s}, 2 \mathrm{H}), 4.36(\mathrm{~d}, J=2.0 \mathrm{~Hz}, 2 \mathrm{H}), 1.91(\mathrm{dd}, J=6.8,2.0 \mathrm{~Hz}, 3 \mathrm{H}) ;{ }^{13} \mathrm{C}$ NMR (101 MHz, $\left.\mathrm{CDCl}_{3}\right) \delta 139.4,137.8,128.6,128.3,128.0,109.7,89.7,83.4,71.6,58.1,16.2$.

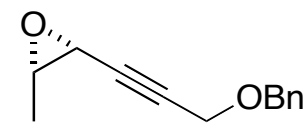

Colorless oil; $[\alpha]_{\mathrm{D}}^{25}=-34.8\left(c 0.31, \mathrm{CHCl}_{3}\right)(92 \%$ ee $) ; \mathrm{IR}(\mathrm{NaCl}): 1348,1074 \mathrm{~cm}^{-1} ;{ }^{1} \mathrm{H} \mathrm{NMR}$ $\left(400 \mathrm{MHz}, \mathrm{CDCl}_{3}\right) \delta$ 7.38-7.28 (m, 5H), $4.61(\mathrm{~s}, 2 \mathrm{H}), 4.24(\mathrm{~d}, J=1.6 \mathrm{~Hz}, 2 \mathrm{H}), 3.50(\mathrm{dt}, J=4.0$, $1.6 \mathrm{~Hz}, 1 \mathrm{H}), 3.20(\mathrm{qd}, J=5.6,4.0 \mathrm{~Hz}, 1 \mathrm{H}), 1.46(\mathrm{~d}, J=5.6 \mathrm{~Hz}, 3 \mathrm{H}) ;{ }^{13} \mathrm{C} \mathrm{NMR}(101 \mathrm{MHz}$, $\left.\mathrm{CDCl}_{3}\right) \delta 137.4,128.7,128.3,128.2,81.7,71.8,57.5,54.2,45.6,15.0$. Anal. Calcd. for $\mathrm{C}_{13} \mathrm{H}_{14} \mathrm{O}_{2}$ : C, 77.20; H, 6.98. Found: C, 77.42; H, 6.70.

\section{Table 1, entry 4}

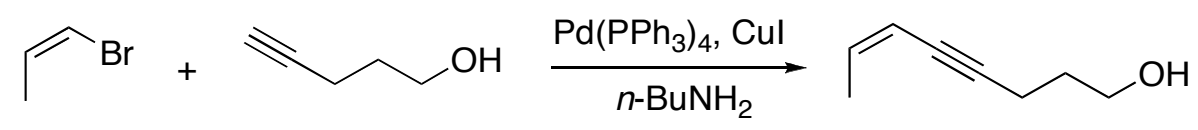

The enyne was prepared via Sonogashira coupling of cis-1-bromo-1-propene with 4-pentyn-1-ol (77\%): Yellow oil; IR (NaCl): 3346, 1435, $1055 \mathrm{~cm}^{-1} ;{ }^{1} \mathrm{H}$ NMR (400 MHz, $\left.\mathrm{CDCl}_{3}\right) \delta 5.89$ (dq, $J=10.8,6.8 \mathrm{~Hz}, 1 \mathrm{H}), 5.48-5.42(\mathrm{~m}, 1 \mathrm{H}), 3.77(\mathrm{t}, J=6.4 \mathrm{~Hz}, 2 \mathrm{H}), 2.47(\mathrm{td}, J=6.8,2.4 \mathrm{~Hz}, 2 \mathrm{H})$, $1.83(\mathrm{dd}, J=6.8,1.6 \mathrm{~Hz}, 3 \mathrm{H}), 1.81-1.78(\mathrm{~m}, 3 \mathrm{H}) ;{ }^{13} \mathrm{C} \mathrm{NMR}\left(101 \mathrm{MHz}, \mathrm{CDCl}_{3}\right) \delta 137.4,110.3$, 94.0, 77.8, 61.8, 31.6, 16.2, 15.9. 


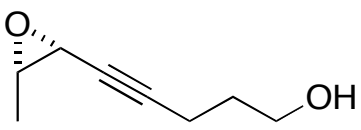

Colorless oil; $[\alpha]^{25}=-27.9\left(c 2.3, \mathrm{CHCl}_{3}\right)(88 \%$ ee $) ; \mathrm{IR}(\mathrm{NaCl}): 3384,2241,1350,1055 \mathrm{~cm}^{-1}$; ${ }^{1} \mathrm{H}$ NMR $\left(400 \mathrm{MHz}, \mathrm{CDCl}_{3}\right) \delta 3.76(\mathrm{q}, J=6.0 \mathrm{~Hz}, 2 \mathrm{H}), 3.42(\mathrm{dt}, J=4.0,2.0 \mathrm{~Hz}, 1 \mathrm{H}), 3.14(\mathrm{qd}, J$ $=5.6,4.0 \mathrm{~Hz}, 1 \mathrm{H}), 2.38(\mathrm{td}, J=7.2,2.0 \mathrm{~Hz}, 2 \mathrm{H}), 1.79$ (quint., $J=6.0 \mathrm{~Hz}, 2 \mathrm{H}), 1.42(\mathrm{~d}, J=5.6$ $\mathrm{Hz}, 3 \mathrm{H}) ;{ }^{13} \mathrm{C} \mathrm{NMR}\left(101 \mathrm{MHz}, \mathrm{CDCl}_{3}\right) \delta 85.9,75.7,61.7,54.2,46.0,31.3,15.5,14.9 . \quad$ Anal. Calcd. for $\mathrm{C}_{8} \mathrm{H}_{12} \mathrm{O}_{2}$ : C, 68.54; H, 8.63. Found: C, 68.37; H, 8.68.

\section{Table 1, entry 5}

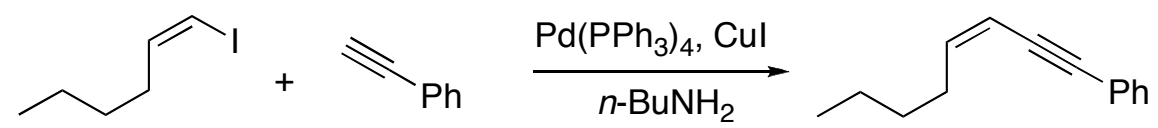

The enyne was prepared via Sonogashira coupling of cis-1-iodo-1-hexene* and phenylacetylene (76\%): Colorless oil; IR (NaCl): $1466 \mathrm{~cm}^{-1} ;{ }^{1} \mathrm{H}$ NMR (400 MHz, $\left.\mathrm{CDCl}_{3}\right) \delta$ 7.36-7.32 (m, 2H), 7.25-7.19 (m, 3H), $5.89(\mathrm{dt}, J=10.8,7.6 \mathrm{~Hz}, 1 \mathrm{H}), 5.58(\mathrm{dt}, J=10.8,1.2 \mathrm{~Hz}, 1 \mathrm{H}), 2.32(\mathrm{qd}, J=$

7.6, $1.2 \mathrm{~Hz}, 2 \mathrm{H}), 1.38-1.26(\mathrm{~m}, 4 \mathrm{H}), 0.85(\mathrm{t}, J=7.2 \mathrm{~Hz}, 3 \mathrm{H}) ;{ }^{13} \mathrm{C} \mathrm{NMR}\left(101 \mathrm{MHz}, \mathrm{CDCl}_{3}\right) \delta$ $144.6,131.6,128.5,128.2,123.9,109.2,93.6,86.7,31.2,30.3,22.5,14.1$.

* Prepared according to the reported procedure: Dieck, H.A.; Heck, R.F. J. Org. Chem. 1975, 40, 1083.

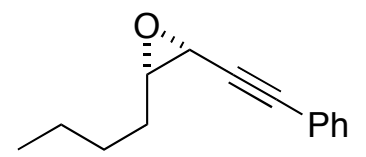

Colorless oil; $[\alpha]_{D}^{25}=-3.6\left(c 0.42, \mathrm{CHCl}_{3}\right)(84 \%$ ee $) ; \mathrm{IR}(\mathrm{NaCl}): 2227,1491 \mathrm{~cm}^{-1} ;{ }^{1} \mathrm{H}$ NMR $\left(400 \mathrm{MHz}, \mathrm{CDCl}_{3}\right) \delta$ 7.48-7.44 (m, 2H), 7.36-7.29 (m, 3H), $3.66(\mathrm{~d}, J=4.4 \mathrm{~Hz}, 1 \mathrm{H}), 3.14(\mathrm{td}, J$ $=6.0,4.4 \mathrm{~Hz}, 1 \mathrm{H}), 1.87-1.72(\mathrm{~m}, 2 \mathrm{H}), 1.59-1.40(\mathrm{~m}, 4 \mathrm{H}), 0.96(\mathrm{t}, J=7.2 \mathrm{~Hz}, 3 \mathrm{H}) ;{ }^{13} \mathrm{C} \mathrm{NMR}$ 
$\left(101 \mathrm{MHz}, \mathrm{CDCl}_{3}\right) \delta 132.1,128.9,128.5,122.4,85.4,84.5,58.9,45.9,29.4,28.3,22.7,14.3$.

Anal. Calcd. for $\mathrm{C}_{14} \mathrm{H}_{16} \mathrm{O}:$ C, 83.96; H, 8.05. Found: C, 84.04; H, 7.78.

Table 1, entry 6

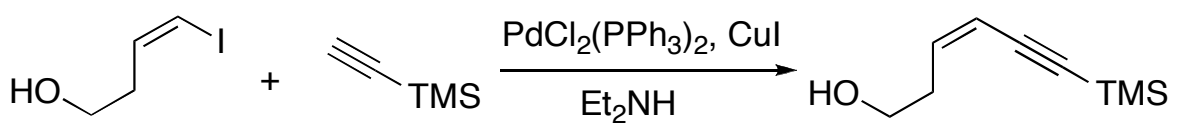

The enyne was prepared via Sonogashira coupling via a slightly different procedure than before:* to a solution of cis-4-iodo-3-buten-1-ol** (2.04 g, $10.3 \mathrm{mmol})$ in $\mathrm{Et}_{2} \mathrm{NH}$ (10 mL) under Ar at $\mathrm{rt}$ was added $\mathrm{PdCl}_{2}\left(\mathrm{PPh}_{3}\right)_{2}(0.09 \mathrm{~g}, 0.13 \mathrm{mmol}), \mathrm{CuI}(0.49 \mathrm{~g}, 2.6 \mathrm{mmol})$, and trimethylsilylacetylene $(1.01 \mathrm{~g}, 10.3 \mathrm{mmol})$ with stirring. After $2 \mathrm{~h}$ the mixture was diluted with $\mathrm{Et}_{2} \mathrm{O}(50 \mathrm{~mL})$, washed with $\mathrm{H}_{2} \mathrm{O}$, brine, dried $\left(\mathrm{Na}_{2} \mathrm{SO}_{4}\right)$, filtered, and concentrated. The residue was then purified by column (9:1 to 3:2 pet. ether-EtOAc) to yield the enyne as a colorless oil (1.38 g, 80\%): IR (NaCl): 3344, 2149, $1250 \mathrm{~cm}^{-1} ;{ }^{1} \mathrm{H}$ NMR (400 MHz, $\left.\mathrm{CDCl}_{3}\right) \delta 5.97(\mathrm{dt}, J=$ 10.8, 7.2 Hz, 1H), $5.59(\mathrm{~d}, J=10.8 \mathrm{~Hz}, 1 \mathrm{H}), 3.71(\mathrm{t}, J=6.8 \mathrm{~Hz}, 2 \mathrm{H}), 2.58(\mathrm{dt}, J=7.2,6.8 \mathrm{~Hz}$ 2H), $1.36(\mathrm{~s}, 1 \mathrm{H}), 0.17(\mathrm{~s}, 9 \mathrm{H}) ;{ }^{13} \mathrm{C}$ NMR $\left(101 \mathrm{MHz}, \mathrm{CDCl}_{3}\right) \delta$ 141.0, 111.9, 101.8, 99.7, 62.0, 34.0, 0.2.

*Marshall, J.A.; Bourbeau, M.P. Org. Lett. 2002, 4, 3931.

**Prepared according to the reported procedure: Denmark, S.E.; Yang, S.M. J. Am. Chem. Soc. 2002, 124, 2102.<smiles>CS(=O)(=O)C#CC=CCCO</smiles>

Colorless oil (note: epoxide should be purified by column without buffering with $\mathrm{Et}_{3} \mathrm{~N}$ ); $[\alpha]_{\mathrm{D}}^{25}=$ $-42.6\left(c\right.$ 0.31, $\left.\mathrm{CHCl}_{3}\right)\left(94 \%\right.$ ee); IR $(\mathrm{NaCl}): 3404,2173,1251 \mathrm{~cm}^{-1} ;{ }^{1} \mathrm{H}$ NMR $(400 \mathrm{MHz}$, $\left.\mathrm{CDCl}_{3}\right) \delta 3.90(\mathrm{td}, J=6.0,1.6 \mathrm{~Hz}, 2 \mathrm{H}), 3.48(\mathrm{~d}, J=4.0,1 \mathrm{H}), 3.26-3.22(\mathrm{~m}, 1 \mathrm{H}), 2.08-1.92(\mathrm{~m}$, 
2H), $0.19(\mathrm{~s}, 9 \mathrm{H}) ;{ }^{13} \mathrm{C}$ NMR $\left(101 \mathrm{MHz}, \mathrm{CDCl}_{3}\right) \delta 100.3,92.0,60.2,56.2,45.2,32.5,-0.1$. Anal. Calcd. for $\mathrm{C}_{9} \mathrm{H}_{16} \mathrm{O}_{2} \mathrm{Si}:$ C, 58.65; H, 8.75. Found: C, 58.90; H, 8.90.

Mukai, C.; Sugimoto, Y-i.; Ikeda, Y.; Hanaoka, M. Chem. Commun. 1994, 1161.

Table 1, entry 7

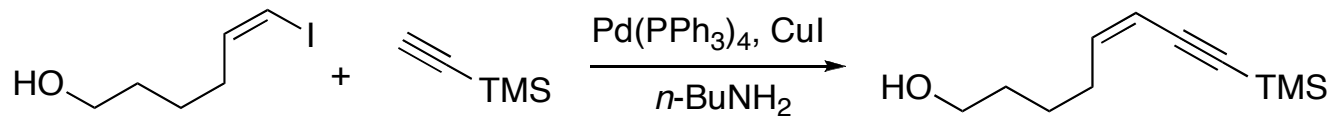

The enyne was prepared via Sonogashira coupling of cis-6-iodo-5-hexen-1-ol* and trimethylsilylacetylene (62\%): Yellow oil; IR (NaCl): 3346, 2150, $1250 \mathrm{~cm}^{-1} ;{ }^{1} \mathrm{H}$ NMR (400 $\left.\mathrm{MHz}, \mathrm{CDCl}_{3}\right) \delta 5.95(\mathrm{dt}, J=10.8,7.6 \mathrm{~Hz}, 1 \mathrm{H}), 5.51(\mathrm{dt}, J=10.8,1.6 \mathrm{~Hz}, 1 \mathrm{H}), 3.68(\mathrm{t}, J=6.4$ $\mathrm{Hz}, 2 \mathrm{H}), 2.37(\mathrm{qd}, J=7.6,1.6 \mathrm{~Hz}, 2 \mathrm{H}), 1.66-1.47(\mathrm{~m}, 4 \mathrm{H}), 1.36(\mathrm{~s}, 1 \mathrm{H}), 0.21(\mathrm{~s}, 9 \mathrm{H}) ;{ }^{13} \mathrm{C} \mathrm{NMR}$ $\left(101 \mathrm{MHz}, \mathrm{CDCl}_{3}\right) \delta 145.1,109.8,102.2,99.0,62.9,32.3,30.1,25.0,0.2$.

* Prepared according to the reported procedure: Denmark, S.E.; Yang, S.M. J. Am. Chem. Soc. 2002, 124, 2102.

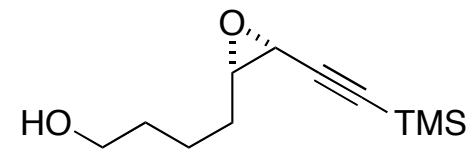

Colorless oil; $[\alpha]_{\mathrm{D}}^{25}=-20.6\left(c 0.35, \mathrm{CHCl}_{3}\right)(87 \%$ ee $)$, IR $(\mathrm{NaCl}): 3383,2176,1251 \mathrm{~cm}^{-1} ;{ }^{1} \mathrm{H}$ NMR (400 MHz, $\left.\mathrm{CDCl}_{3}\right) \delta 3.69(\mathrm{q}, J=6.4 \mathrm{~Hz}, 2 \mathrm{H}), 3.43(\mathrm{~d}, J=4.0 \mathrm{~Hz}, 1 \mathrm{H}), 3.04(\mathrm{td}, J=6.0$, $4.0 \mathrm{~Hz}, 1 \mathrm{H}), 1.83-1.56(\mathrm{~m}, 6 \mathrm{H}), 0.19(\mathrm{~s}, 9 \mathrm{H}) ;{ }^{13} \mathrm{C} \mathrm{NMR}\left(101 \mathrm{MHz}, \mathrm{CDCl}_{3}\right) \delta 100.5,91.5,62.9$, 58.3, 45.5, 32.6, 29.2, 22.3, -0.1. Anal. Calcd. for $\mathrm{C}_{11} \mathrm{H}_{20} \mathrm{O}_{2} \mathrm{Si}$ : C, 62.21; H, 9.49. Found: C, 62.20; H, 9.41. 
Table 1, entry 8

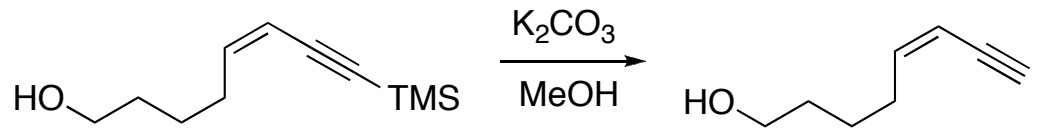

The desilylation was carried out according to the reported method:* cis-8-Trimethylsilyloct-5en-7-yn-1-ol $(0.71 \mathrm{~g}, 3.4 \mathrm{mmol})$ was treated with saturated methanolic $\mathrm{K}_{2} \mathrm{CO}_{3}$ solution $(10.6 \mathrm{~mL})$ and stirred at $\mathrm{rt}$ until the starting material disappeared as judged by TLC. The mixture was then extracted with $\mathrm{Et}_{2} \mathrm{O}$, and the combined organic layers were washed with brine, dried $\left(\mathrm{Na}_{2} \mathrm{SO}_{4}\right)$, filtered and concentrated. The residue was purified by column (1:2 pet. ether-Et $\left.{ }_{2} \mathrm{O}\right)$ to yield the desilylated enyne as a colorless oil $(0.42 \mathrm{~g}, 48 \%)$ : IR (NaCl): 3289, 2096, $1066 \mathrm{~cm}^{-1}$; ${ }^{1} \mathrm{H}$ NMR $\left(400 \mathrm{MHz}, \mathrm{CDCl}_{3}\right) \delta 6.01(\mathrm{dt}, J=10.8,7.2 \mathrm{~Hz}, 1 \mathrm{H}), 5.50-5.46(\mathrm{~m}, 1 \mathrm{H}), 3.68(\mathrm{t}, J=6.0 \mathrm{~Hz}, 2 \mathrm{H})$, $3.09(\mathrm{~d}, J=2.0 \mathrm{~Hz}, 1 \mathrm{H}), 2.38(\mathrm{qd}, J=7.2,1.2 \mathrm{~Hz}, 2 \mathrm{H}), 1.66-1.58(\mathrm{~m}, 2 \mathrm{H}), 1.56-1.47(\mathrm{~m}, 2 \mathrm{H})$, $1.40(\mathrm{~s}, 1 \mathrm{H}) ;{ }^{13} \mathrm{C} \mathrm{NMR}\left(101 \mathrm{MHz}, \mathrm{CDCl}_{3}\right) \delta 145.8,108.7,81.6,80.7,62.9,32.3,30.1,25.1$. * Cai, C.; Vasella, A. Helv. Chim. Acta 1995, 78, 732.

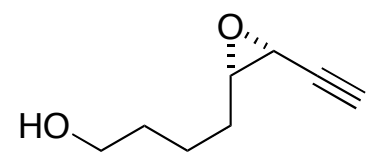

Colorless oil; $[\alpha]_{\mathrm{D}}^{25}=-40.0\left(c 0.12, \mathrm{CHCl}_{3}\right)(80 \%$ ee $) ; \mathrm{IR}(\mathrm{NaCl}): 3382,3291,2120 \mathrm{~cm}^{-1} ;{ }^{1} \mathrm{H}$ NMR (400 MHz, $\left.\mathrm{CDCl}_{3}\right) \delta 3.69(\mathrm{t}, J=6.0 \mathrm{~Hz}, 2 \mathrm{H}), 3.43(\mathrm{dd}, J=4.0,1.8 \mathrm{~Hz}, 1 \mathrm{H}), 3.05(\mathrm{td}, J=$ 6.0, 4.0 Hz, 1H), $2.36(\mathrm{~d}, J=1.8 \mathrm{~Hz}, 1 \mathrm{H}), 1.82-1.56(\mathrm{~m}, 6 \mathrm{H}), 1.42$ (brs, $1 \mathrm{H}) ;{ }^{13} \mathrm{C}$ NMR $(101$ $\left.\mathrm{MHz}, \mathrm{CDCl}_{3}\right) \delta 79.1,73.9,62.8,57.9,44.9,32.5,29.1,22.4$. Anal. Calcd. for $\mathrm{C}_{8} \mathrm{H}_{12} \mathrm{O}_{2}$ : C, 68.54; H, 8.63. Found: C, 68.38; H, 8.39. 
Table 1, entry 9

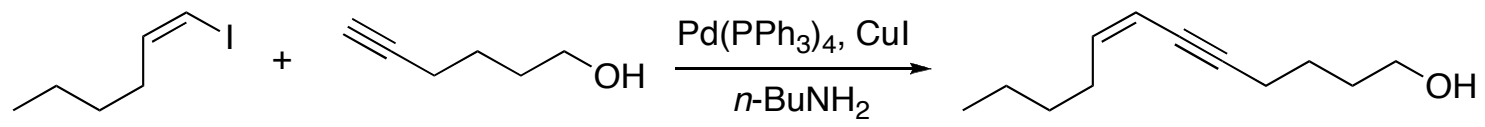

The enyne was prepared via Sonogashira coupling of cis-1-iodo-1-hexene* and 5-hexyn-1-ol (71\%): Orange oil; IR (NaCl): 3344, 2213, 1721, $1061 \mathrm{~cm}^{-1} ;{ }^{1} \mathrm{H}$ NMR $\left(400 \mathrm{MHz}, \mathrm{CDCl}_{3}\right) \delta$ $5.83(\mathrm{dt}, J=10.8,7.2 \mathrm{~Hz}, 1 \mathrm{H}), 5.46-5.41(\mathrm{~m}, 1 \mathrm{H}), 3.70(\mathrm{t}, J=6.2 \mathrm{~Hz}, 2 \mathrm{H}), 2.40(\mathrm{td}, J=6.8,2.0$ $\mathrm{Hz}, 2 \mathrm{H}), 2.30(\mathrm{q}, J=7.2 \mathrm{~Hz}, 2 \mathrm{H}), 1.76-1.60(\mathrm{~m}, 4 \mathrm{H}), 1.43-1.32(\mathrm{~m}, 4 \mathrm{H}), 0.92(\mathrm{t}, J=6.8, \mathrm{~Hz}$, $3 \mathrm{H}) ;{ }^{13} \mathrm{C} \mathrm{NMR}\left(101 \mathrm{MHz}, \mathrm{CDCl}_{3}\right) \delta 143.1,109.4,94.0,78.0,62.7,32.1,31.2,30.0,25.3,22.5$, 19.5, 14.1.

* Dieck, H.A.; Heck, R.F. J. Org. Chem. 1975, 40, 1083.

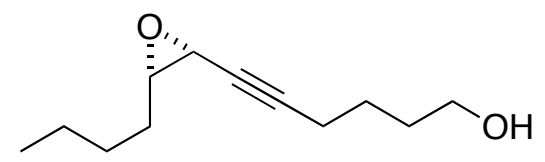

Colorless oil; $[\alpha]^{25}=-14.1\left(c 0.26, \mathrm{CHCl}_{3}\right)(80 \%$ ee $)$; IR $(\mathrm{NaCl}): 3385,2240,1457,1061$ $\mathrm{cm}^{-1} ;{ }^{1} \mathrm{H}$ NMR $\left(400 \mathrm{MHz}, \mathrm{CDCl}_{3}\right) \delta 3.68(\mathrm{t}, J=6.4 \mathrm{~Hz}, 2 \mathrm{H}), 3.42(\mathrm{dt}, J=4.4,2.0 \mathrm{~Hz}, 1 \mathrm{H}), 3.00$ $(\mathrm{td}, J=6.0,4.4 \mathrm{~Hz}, 1 \mathrm{H}), 2.29(\mathrm{td}, J=6.8,2.0 \mathrm{~Hz}, 2 \mathrm{H}), 1.77-1.36(\mathrm{~m}, 10 \mathrm{H}), 1.26(\mathrm{brs}, 1 \mathrm{H}), 0.94$ $(\mathrm{t}, J=7.2 \mathrm{~Hz}, 3 \mathrm{H}) ;{ }^{13} \mathrm{C} \mathrm{NMR}\left(101 \mathrm{MHz}, \mathrm{CDCl}_{3}\right) \delta 86.2,75.7,62.6,58.3,45.7,32.0,29.2,28.3$, 24.9, 22.7, 18.8, 14.2. Anal. Calcd. for $\mathrm{C}_{12} \mathrm{H}_{20} \mathrm{O}_{2}$ : C, 73.43; H, 10.27. Found: C, 73.19; H, 10.26

Table 1, entry 10

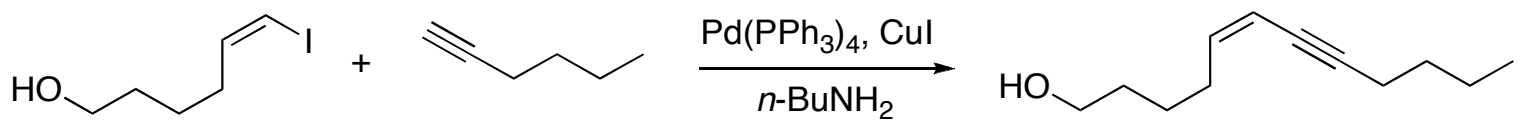


The enyne was prepared via Sonogashira coupling of cis-6-iodo-5-hexen-1-ol* and 1-hexyne (63\%): Orange oil; IR (NaCl): 3333, 2210, 1458, $1061 \mathrm{~cm}^{-1} ;{ }^{1} \mathrm{H}$ NMR $\left(400 \mathrm{MHz}, \mathrm{CDCl}_{3}\right) \delta$ $5.81(\mathrm{dt}, J=10.8,7.4 \mathrm{~Hz}, 1 \mathrm{H}), 5.49-5.44(\mathrm{~m}, 1 \mathrm{H}), 3.67(\mathrm{t}, J=6.6 \mathrm{~Hz}, 2 \mathrm{H}), 2.37-2.30(\mathrm{~m}, 4 \mathrm{H})$, 1.66-1.28 (m, 8H), $0.93(\mathrm{t}, J=7.2 \mathrm{~Hz}, 3 \mathrm{H}) ;{ }^{13} \mathrm{C} \mathrm{NMR}\left(101 \mathrm{MHz}, \mathrm{CDCl}_{3}\right) \delta 142.1,110.1,94.9$, $77.4,63.0,32.4,31.2,29.8,25.2,22.2,19.4,13.8$.

* Prepared according to the reported procedure: Denmark, S.E.; Yang, S.M. J. Am. Chem. Soc. 2002, 124, 2102.

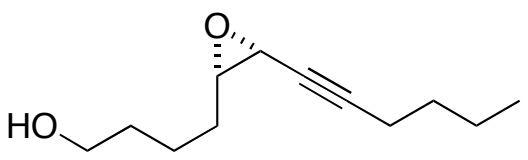

Colorless oil; $[\alpha]^{25}=-8.2\left(c 0.65, \mathrm{CHCl}_{3}\right)(90 \%$ ee $) ; \mathrm{IR}(\mathrm{NaCl}): 3358,2239,1458 \mathrm{~cm}^{-1} ;{ }^{1} \mathrm{H}$ NMR (400 MHz, $\left.\mathrm{CDCl}_{3}\right) \delta 3.69(\mathrm{q}, J=6.0 \mathrm{~Hz}, 2 \mathrm{H}), 3.43(\mathrm{dt}, J=4.0,1.6 \mathrm{~Hz}, 2 \mathrm{H}), 3.01(\mathrm{td}, J=$ 6.0, 4.0 Hz, 1H), $2.23(\mathrm{td}, J=6.8,1.6 \mathrm{~Hz}, 2 \mathrm{H}), 1.81-1.28(\mathrm{~m}, 10 \mathrm{H}), 0.91(\mathrm{t}, J=7.2 \mathrm{~Hz}, 3 \mathrm{H}) ;{ }^{13} \mathrm{C}$ NMR (101 MHz, $\left.\mathrm{CDCl}_{3}\right) \delta 86.9,75.1,62.9,58.1,45.8,32.6,30.7,29.2,22.4,22.1,18.7,13.8$. Anal. Calcd. for $\mathrm{C}_{12} \mathrm{H}_{20} \mathrm{O}_{2}$ : C, 73.43; H, 10.27. Found: C, 73.14; H, 10.15.

\section{Table 1, entry 11}

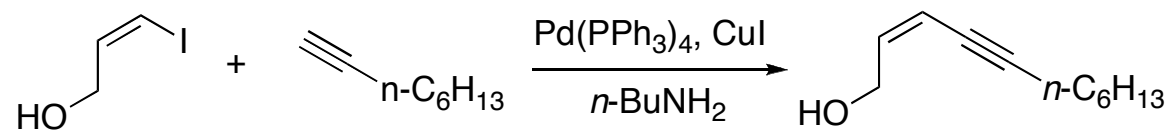

The enyne was prepared via Sonogashira coupling of cis-3-iodo-2-propen-1-ol* and 1-octyne (85\%): Orange oil; IR (NaCl): 3326, 2216, $1020 \mathrm{~cm}^{-1} ;{ }^{1} \mathrm{H}$ NMR $\left(400 \mathrm{MHz}, \mathrm{CDCl}_{3}\right) \delta 6.01(\mathrm{dt}$, $J=11.0,6.4 \mathrm{~Hz}, 1 \mathrm{H}), 5.61-5.56(\mathrm{~m}, 1 \mathrm{H}), 4.40(\mathrm{dd}, J=6.4,1.2 \mathrm{~Hz}, 2 \mathrm{H}), 2.34(\mathrm{td}, J=6.8,2.0 \mathrm{~Hz}$ $2 \mathrm{H}), 1.63(\mathrm{~s}, 1 \mathrm{H}), 1.58-1.51(\mathrm{~m}, 2 \mathrm{H}), 1.44-1.26(\mathrm{~m}, 6 \mathrm{H}), 0.90(\mathrm{t}, J=7.2 \mathrm{~Hz}, 3 \mathrm{H}) ;{ }^{13} \mathrm{C} \mathrm{NMR}(101$ $\left.\mathrm{MHz}, \mathrm{CDCl}_{3}\right) \delta 140.0,111.5,97.1,76.4,61.2,31.5,28.9,28.8,22.8,19.7,14.2$. 
*Prepared according to the reported procedure: Denmark, S.E.; Yang, S.M. J. Am. Chem. Soc. 2002, 124, 2102.<smiles>OCCCCCC=CC#CC1OC1CO</smiles>

Colorless oil (note: epoxide should be purified by column without buffering with $\mathrm{Et}_{3} \mathrm{~N}$ ); $[\alpha]_{\mathrm{D}}^{25}=$ $-41.4\left(c 0.22, \mathrm{CHCl}_{3}\right)\left(97 \%\right.$ ee); IR (NaCl): 3416, 2234, $1044 \mathrm{~cm}^{-1} ;{ }^{1} \mathrm{H}$ NMR (400 MHz, $\left.\mathrm{CDCl}_{3}\right) \delta 3.98-3.89(\mathrm{~m}, 1 \mathrm{H}), 3.88-3.82(\mathrm{~m}, 1 \mathrm{H}), 3.54(\mathrm{dt}, J=4.4,1.6 \mathrm{~Hz}, 1 \mathrm{H}), 3.26(\mathrm{td}, J=6.0$, $4.4 \mathrm{~Hz}, 1 \mathrm{H}), 2.22(\mathrm{td}, J=7.2,1.6 \mathrm{~Hz}, 2 \mathrm{H}), 1.70$ (brs, $1 \mathrm{H}), 1.52$ (quint., $J=7.2 \mathrm{~Hz}, 2 \mathrm{H}$ ), 1.44 $1.24(\mathrm{~m}, 6 \mathrm{H}), 0.90(\mathrm{t}, J=6.8 \mathrm{~Hz}, 3 \mathrm{H}) ;{ }^{13} \mathrm{C} \mathrm{NMR}\left(101 \mathrm{MHz}, \mathrm{CDCl}_{3}\right) \delta 87.7,74.3,62.4,57.4$, 44.9, 31.5, 28.7, 28.5, 22.7, 19.0, 14.2. Anal. Calcd. for $\mathrm{C}_{11} \mathrm{H}_{18} \mathrm{O}_{2}$ : C, 72.49; $\mathrm{H}$, 9.95. Found: C, 72.66; H, 9.71.

Table 1, entry 12

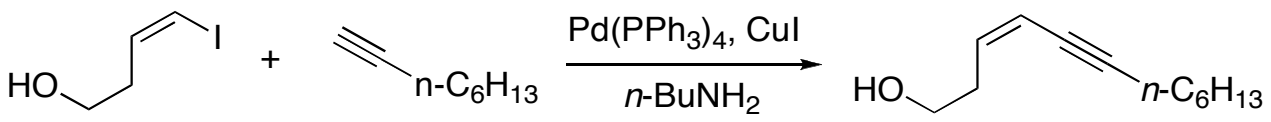

The enyne was prepared via Sonogashira coupling of cis-4-iodo-3-buten-1-ol* and 1-octyne (83\%): Orange oil; IR (NaCl): 3330, 2217, $1048 \mathrm{~cm}^{-1} ;{ }^{1} \mathrm{H}$ NMR (400 MHz, $\left.\mathrm{CDCl}_{3}\right) \delta 5.87(\mathrm{dt}$, $J=10.8,7.2 \mathrm{~Hz}, 1 \mathrm{H}), 5.64-5.58(\mathrm{~m}, 1 \mathrm{H}), 3.73(\mathrm{q}, J=6.0 \mathrm{~Hz}, 2 \mathrm{H}), 2.58(\mathrm{qd}, J=6.4,1.2 \mathrm{~Hz}, 2 \mathrm{H})$, $2.34(\mathrm{td}, J=7.2,2.0 \mathrm{~Hz}, 2 \mathrm{H}), 1.59-1.51(\mathrm{~m}, 2 \mathrm{H}), 1.45-1.27(\mathrm{~m}, 6 \mathrm{H}), 0.90(\mathrm{t}, J=7.2 \mathrm{~Hz}, 3 \mathrm{H}) ;{ }^{13} \mathrm{C}$ $\operatorname{NMR}\left(101 \mathrm{MHz}, \mathrm{CDCl}_{3}\right) \delta 137.9,112.5,95.6,62.2,33.8,31.5,29.0,28.8,22.8,19.7,14.3$.

*Prepared according to the reported procedure: Denmark, S.E.; Yang, S.M. J. Am. Chem. Soc. 2002, 124, 2102. 


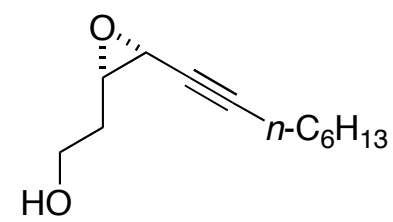

Colorless oil (note: epoxide should be purified by column without buffering with $\mathrm{Et}_{3} \mathrm{~N}$ ); $[\alpha]^{25}{ }_{\mathrm{D}}=$ $-36.8\left(c\right.$ 0.37, $\left.\mathrm{CHCl}_{3}\right)\left(97 \%\right.$ ee); IR $(\mathrm{NaCl}): 3418,2237,1051 \mathrm{~cm}^{-1} ;{ }^{1} \mathrm{H}$ NMR $(400 \mathrm{MHz}$, $\left.\mathrm{CDCl}_{3}\right) \delta 3.92-3.88(\mathrm{~m}, 2 \mathrm{H}), 3.48(\mathrm{dt}, J=6.0,1.6 \mathrm{~Hz}, 1 \mathrm{H}), 3.23-3.19(\mathrm{~m}, 1 \mathrm{H}), 2.23(\mathrm{td}, J=7.2$, $1.6 \mathrm{~Hz}, 2 \mathrm{H}), 2.06-1.91(\mathrm{~m}, 2 \mathrm{H}), 1.62-1.24(\mathrm{~m}, 8 \mathrm{H}) ,0.90(\mathrm{t}, J=7.2 \mathrm{~Hz}, 3 \mathrm{H}) ;{ }^{13} \mathrm{C}$ NMR $(101$ $\left.\mathrm{MHz}, \mathrm{CDCl}_{3}\right) \delta 87.5,75.0,60.4,56.0,45.4,32.5,31.5,28.7,28.6,22.7,19.0,14.2$. Anal. Calcd. for $\mathrm{C}_{12} \mathrm{H}_{20} \mathrm{O}_{2}$ : C, 73.43; H, 10.27. Found: C, 73.19; H, 10.07.

Table 1, entry 13

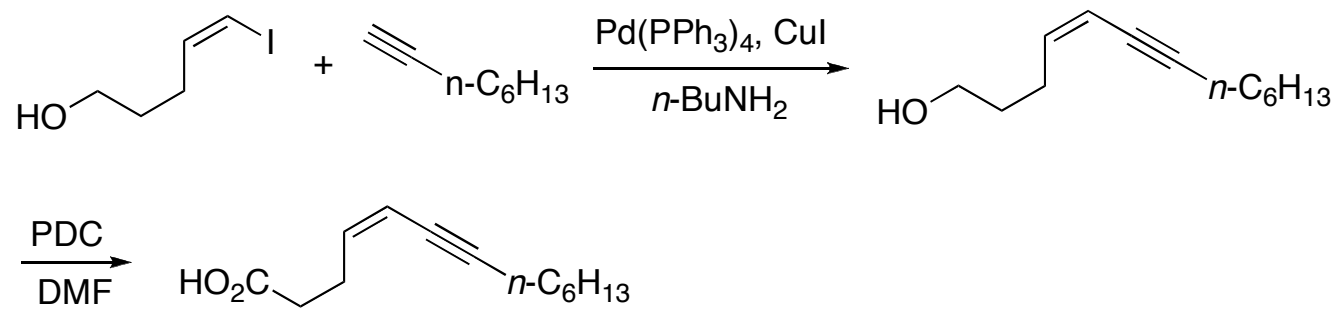

The enyne was prepared via Sonogashira coupling of 5-iodo-4-penten-1-ol* and 1-octyne. The crude alcohol was taken on to the next step without purification: Brown oil; IR ( $\mathrm{NaCl})$ : 3340, 2211, $1456 \mathrm{~cm}^{-1} ;{ }^{1} \mathrm{H}$ NMR (400 MHz, $\left.\mathrm{CDCl}_{3}\right) \delta 5.83(\mathrm{dt}, J=10.4,7.2 \mathrm{~Hz}, 1 \mathrm{H}), 5.51(\mathrm{~m}, 1 \mathrm{H})$, $3.67(\mathrm{t}, J=6.4 \mathrm{~Hz}, 2 \mathrm{H}), 2.40(\mathrm{q}, J=7.2 \mathrm{~Hz}, 2 \mathrm{H}), 2.34(\mathrm{td}, J=7.22 .0 \mathrm{~Hz}, 2 \mathrm{H}), 1.69$ (quint. $J=$ $6.8 \mathrm{~Hz}, 2 \mathrm{H}), 1.55$ (quint., $J=7.6 \mathrm{~Hz}, 2 \mathrm{H}), 1.45-1.37(\mathrm{~m}, 2 \mathrm{H}), 1.37-1.24(\mathrm{~m}, 4 \mathrm{H}), 0.90(\mathrm{t}, J=6.8$ $\mathrm{Hz}, 3 \mathrm{H}) ;{ }^{13} \mathrm{C} \mathrm{NMR}\left(101 \mathrm{MHz}, \mathrm{CDCl}_{3}\right) \delta 141.4,110.6,95.4,94.6,62.3,31.7,31.6,29.0,28.8$, $26.3,22.8,19.7,14.3$.

*Prepared according to the reported procedure: Denmark, S.E.; Yang, S.M. J. Am. Chem. Soc. 2002, 124, 2102. 
The oxidation was carried out according to the reported method.* To a solution of cis-tridec-4en-6-yn-1-ol (3.7 g, $21 \mathrm{mmol})$ in DMF (57 mL) was added PDC (26.5 g, $70.4 \mathrm{mmol})$ at rt with stirring under Ar. The mixture was stirred overnight and then filtered through a pad of silica gel while washing with $\mathrm{Et}_{2} \mathrm{O}$. The filtrate was concentrated and the residue was purified by column (7:1 hexanes- $\mathrm{Et}_{2} \mathrm{O}$ to $\left.\mathrm{Et}_{2} \mathrm{O}\right)$ to yield the acid as a colorless oil (2.21 g, $50 \%$ over two steps): IR (NaCl): 2215, $1712 \mathrm{~cm}^{-1} ;{ }^{1} \mathrm{H}$ NMR (400 MHz, $\left.\mathrm{CDCl}_{3}\right) \delta 11.14$ (brs, 1H), 5.84 (dt, J = 10.8, 7.2 $\mathrm{Hz}, 1 \mathrm{H}), 5.52(\mathrm{~m}, 1 \mathrm{H}), 2.62(\mathrm{q}, J=7.2 \mathrm{~Hz}, 2 \mathrm{H}), 2.49(\mathrm{~m}, 2 \mathrm{H}), 2.35(\mathrm{td}, J=6.8,2.0 \mathrm{~Hz}, 2 \mathrm{H}), 1.55$ (quint., $J=7.6 \mathrm{~Hz}, 2 \mathrm{H}), 1.46-1.36(\mathrm{~m}, 2 \mathrm{H}), 1.36-1.24(\mathrm{~m}, 4 \mathrm{H}), 0.90(\mathrm{t}, J=7.2 \mathrm{~Hz}, 3 \mathrm{H}) ;{ }^{13} \mathrm{C}$ NMR $\left(101 \mathrm{MHz}, \mathrm{CDCl}_{3}\right) \delta 178.9,139.4,111.5,96.1,33.3,31.6,29.0,28.8,25.3,22.8,19.8$, 14.3.

* Corey, E. J.; Schmidt, G. Tetrahedron Lett. 1979, 399.

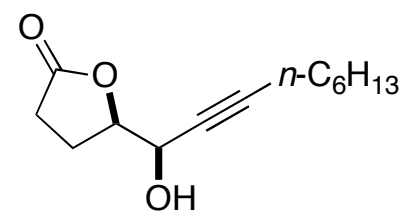

Colorless oil; $[\alpha]_{\mathrm{D}}^{25}=-19.3\left(c 0.29, \mathrm{CHCl}_{3}\right)(96 \%$ ee $) ; \mathrm{IR}(\mathrm{NaCl}): 3427,2233,1778 \mathrm{~cm}^{-1} ;{ }^{1} \mathrm{H}$ NMR (400 MHz, $\left.\mathrm{CDCl}_{3}\right) \delta 4.56(\mathrm{ddd}, J=7.6,6.0,5.6 \mathrm{~Hz}, 1 \mathrm{H}), 4.46(\mathrm{~m}, 1 \mathrm{H}), 2.70-2.50(\mathrm{~m}, 2 \mathrm{H})$, 2.40-2.30 (m, 1H), 2.26-2.14 (m, 4H), 1.56-1.47 (m, 2H), 1.42-1.23 (m, 6H), 0.90 (t, J = 7.2 Hz, $1 \mathrm{H}) ;{ }^{13} \mathrm{C} \mathrm{NMR}\left(101 \mathrm{MHz}, \mathrm{CDCl}_{3}\right) \delta 177.0,88.6,81.9,76.3,65.2,31.5,28.7,28.5,28.4,23.6$, 22.7, 18.9, 14.3. Anal. Calcd for $\mathrm{C}_{13} \mathrm{H}_{20} \mathrm{O}_{3}$ : C, 69.61; H, 8.99. Found: C, 69.39; H, 9.16. 


\section{Table 1, entry 14}

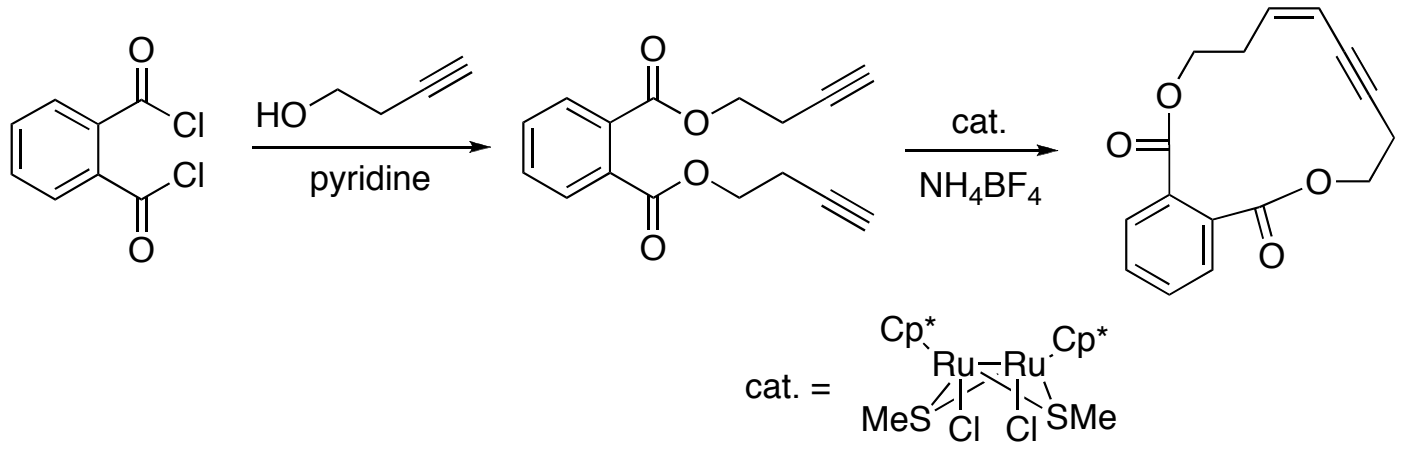

Phthaloyl dichloride (5.1 g, $25.0 \mathrm{mmol})$, 3-butyn-1-ol (3.4 g, $48 \mathrm{mmol})$, and pyridine (7.6 g, 96 mmol) were dissolved in $\mathrm{CH}_{2} \mathrm{Cl}_{2}(250 \mathrm{~mL})$ at $0{ }^{\circ} \mathrm{C}$ with stirring. The mixture was allowed to gradually warm to rt and was stirred overnight. The mixture was then poured into sat. aq. $\mathrm{NH}_{4} \mathrm{Cl}$ and the aq. phase was extracted with $\mathrm{CH}_{2} \mathrm{Cl}_{2}$. The combined organic phases were then washed with $\mathrm{H}_{2} \mathrm{O}$, brine, dried $\left(\mathrm{Na}_{2} \mathrm{SO}_{4}\right)$, filtered, and concentrated. The residue was purified by column (1:1 hexanes- $\left.\mathrm{Et}_{2} \mathrm{O}\right)$ to yield the diester as a colorless syrup $(5.0 \mathrm{~g}, 74 \%): \mathrm{IR}(\mathrm{NaCl}): 3293,2122$, $1728 \mathrm{~cm}^{-1} ;{ }^{1} \mathrm{H}$ NMR $\left(400 \mathrm{MHz}, \mathrm{CDCl}_{3}\right) \delta$ 7.78-7.74 (m, 2H), 7.59-7.53 (m, 2H), $4.43(\mathrm{t}, J=6.8$ $\mathrm{Hz}, 4 \mathrm{H}), 2.66(\mathrm{td}, J=6.8,2.8 \mathrm{~Hz}, 4 \mathrm{H}), 2.04(\mathrm{t}, J=2.8 \mathrm{~Hz}, 2 \mathrm{H}) ;{ }^{13} \mathrm{C} \mathrm{NMR}\left(101 \mathrm{MHz}, \mathrm{CDCl}_{3}\right) \delta$ $167.4,132.0,131.5,129.3,80.1,70.3,63.4,19.1$.

The enyne was prepared according to the method of Nishibayashi et al. ${ }^{*} \mathrm{NH}_{4} \mathrm{BF}_{4}(0.036 \mathrm{~g}, 0.34$ $\mathrm{mmol})$ and phthalic acid dibut-3-ynyl ester $(0.465 \mathrm{~g}, 1.72 \mathrm{mmol})$ were added to a solution of $\left[\mathrm{Cp} * \mathrm{RuCl}\left(\mu_{2}-\mathrm{SMe}\right)_{2} \mathrm{RuCp} * \mathrm{Cl}\right](0.218 \mathrm{~g}, 0.342 \mathrm{mmol})$ in $850 \mathrm{~mL}$ dry $\mathrm{MeOH}$. The reaction was followed by $\mathrm{GC}$ and was stirred at $60{ }^{\circ} \mathrm{C}$ for $24 \mathrm{~h}$ after which time an additional $0.12 \mathrm{~g}(0.19$ mmol) of catalyst was added. After $48 \mathrm{~h}$ more, an additional $0.19 \mathrm{~g}(0.30 \mathrm{mmol})$ of catalyst was added. After stirring for $120 \mathrm{~h}$ total, the solvent was removed and the residue was purified by column $\left(1: 1\right.$ hexanes-Et $\left.{ }_{2} \mathrm{O}\right)$ to yield the enyne as white crystals $(0.39 \mathrm{~g}, 84 \%)$ : $\mathrm{mp} 108-109{ }^{\circ} \mathrm{C}$; IR (NaCl): 2215, 1717, 1275, $1133 \mathrm{~cm}^{-1} ;{ }^{1} \mathrm{H}$ NMR (400 MHz, $\left.\mathrm{CDCl}_{3}\right) \delta$ 7.77-7.68 (m, 2H), 
7.55-7.50 (m, 2H), $5.91(\mathrm{dt}, J=10.4,8.0 \mathrm{~Hz}, 1 \mathrm{H}), 5.54(\mathrm{~d}, J=10.4 \mathrm{~Hz}, 1 \mathrm{H}), 4.54(\mathrm{t}, J=5.2 \mathrm{~Hz}$, 2H), $4.41(\mathrm{t}, J=5.6 \mathrm{~Hz}, 2 \mathrm{H}), 2.82-2.72(\mathrm{~m}, 4 \mathrm{H}) ;{ }^{13} \mathrm{C} \mathrm{NMR}\left(101 \mathrm{MHz}, \mathrm{CDCl}_{3}\right) \delta 168.4,167.2$, $138.9,133.0,132.5,131.3,131.2,129.5,129.0,112.6,90.4,79.2,65.1,62.9,29.9,20.6$.

*Nishibayashi, Y.; Yamanashi, M.; Wakiji, I.; Hidai, M. Angew. Chem. Int. Ed. 2000, 39, 2909.

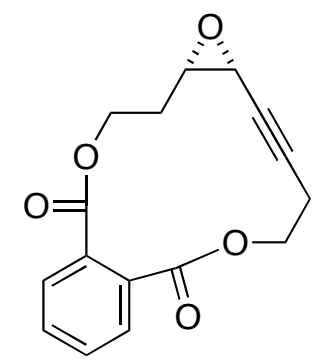

Colorless oil; $[\alpha]_{\mathrm{D}}{ }^{25}=-87.1\left(c 0.86, \mathrm{CHCl}_{3}\right)(87 \%$ ee $)$; IR (NaCl): 2245, 1728, 1293, $1129 \mathrm{~cm}^{-1}$; ${ }^{1} \mathrm{H}$ NMR (400 MHz, $\left.\mathrm{CDCl}_{3}\right)$ d 7.79-7.71 (m, 2H), 7.60-7.53 (m, 2H), 4.84-4.76 (m, 1H), 4.73 (dt, $J=11.2,4.0 \mathrm{~Hz}, 1 \mathrm{H}), 4.28(\mathrm{td}, J=11.2,3.2 \mathrm{~Hz}, 1 \mathrm{H}), 4.17(\mathrm{ddd}, J=10.4,4.8,3.2 \mathrm{~Hz}, 1 \mathrm{H}), 3.44-$ $3.42(\mathrm{~m}, 1 \mathrm{H}), 3.11(\mathrm{ddd}, J=10.0,4.0,1.6 \mathrm{~Hz}, 1 \mathrm{H}), 2.82-2.72(\mathrm{~m}, 1 \mathrm{H}), 2.59(\mathrm{dt}, J=17.6,2.8 \mathrm{~Hz}$, 1H), 2.34-2.26 (m, 1H), 2.23-2.11 (m, 1H); $\left.{ }^{13} \mathrm{C} \mathrm{NMR} \mathrm{(101} \mathrm{MHz,} \mathrm{CDCl}_{3}\right) \delta 168.5,166.9,132.9$, $132.1,131.5,131.3,129.6,129.1,82.9,77.1,63.3,62.3,56.9,46.3,28.3,20.1$. Anal. Calcd for $\mathrm{C}_{16} \mathrm{H}_{14} \mathrm{O}_{5}: \mathrm{C}, 67.13 ; \mathrm{H}, 4.93$. Found: C, 67.36; H, 5.05.

\section{Table 1, entry 15}

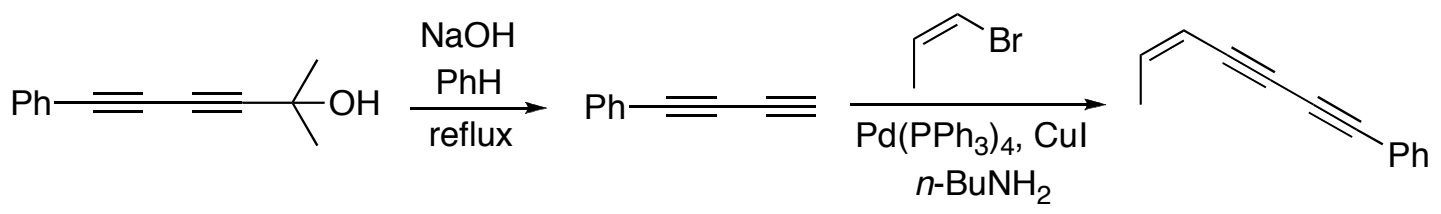

Powdered $\mathrm{NaOH}(0.66 \mathrm{~g}, 16.4 \mathrm{mmol})$ and 6-phenyl-3,5-hexadiyn-2-ol* (2.73 g, $14.8 \mathrm{mmol})$ were added to $29 \mathrm{~mL}$ of toluene and the mixture was refluxed under Ar until the starting material had disappeared by TLC ( $1 \mathrm{~h})$. The brown mixture was then cooled to rt, filtered through a pad 
of $\mathrm{SiO}_{2}$, and washed with 1:1 hexanes- $\mathrm{Et}_{2} \mathrm{O}$. The filtrate was concentrated to give a yellow oil which should be used immediately for the next step.

cis-1-Bromo-1-propene $(3.6 \mathrm{~g}, 29.6 \mathrm{mmol})$ and $\mathrm{Pd}\left(\mathrm{PPh}_{3}\right)_{4}(0.76 \mathrm{~g}, 0.66 \mathrm{mmol})$ were then added to a flask with $33 \mathrm{~mL}$ of benzene. A solution of the above crude terminal diyne in $15 \mathrm{~mL} n$ $\mathrm{BuNH}_{2}(150 \mathrm{mmol})$ was then added to the benzene solution, followed by $\mathrm{CuI}(0.46 \mathrm{~g}, 2.4 \mathrm{mmol})$. The mixture was stirred at $\mathrm{rt}$ under Ar overnight and then hexane $(50 \mathrm{~mL})$ was added. The mixture was poured into sat. aq. $\mathrm{NH}_{4} \mathrm{Cl}$ and washed with sat. aq. $\mathrm{NH}_{4} \mathrm{Cl}, \mathrm{H}_{2} \mathrm{O}$, brine, dried $\left(\mathrm{Na}_{2} \mathrm{SO}_{4}\right)$, filtered, and concentrated. The residue was purified by column (hexanes) to give the endiyne as a colorless oil $(0.88 \mathrm{~g}, 36 \%)$ : IR $(\mathrm{NaCl}): 2214,2149,1490 \mathrm{~cm}^{-1} ;{ }^{1} \mathrm{H}$ NMR (400 $\left.\mathrm{MHz}, \mathrm{CDCl}_{3}\right) \delta$ 7.54-7.46 (m, 2H), 7.41-7.30 (m, 3H), $6.20(\mathrm{dq}, J=10.8,6.8 \mathrm{~Hz}, 1 \mathrm{H}), 5.61(\mathrm{dd}$, $J=10.8,0.8 \mathrm{~Hz}, 1 \mathrm{H}), 1.97(\mathrm{dd}, J=6.8,0.8 \mathrm{~Hz}, 3 \mathrm{H}) ;{ }^{13} \mathrm{C} \mathrm{NMR}\left(101 \mathrm{MHz}, \mathrm{CDCl}_{3}\right) \delta 143.3$, $132.6,129.3,128.6,122.2,109.3,81.9,79.0,78.2,74.2,16.8$.

* (a) Dabdoub, M.J.; Baroni, A.C.M.; Lenardão, E.J.; Gianeti, T.R.; Hurtado, G. Tetrahedron 2001, 57, 4271. (b) Jiang, M. X.-W.; Rawat, M.; Wulff, W.D. J. Am. Chem. Soc. 2004, 126, 5970.

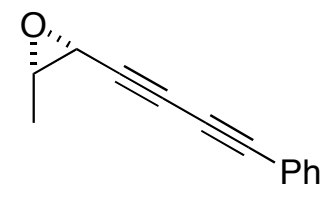

Colorless oil; $[\alpha]_{\mathrm{D}}{ }^{25}=-48.4\left(c 0.25, \mathrm{CHCl}_{3}\right)(93 \%$ ee $) ; \mathrm{IR}(\mathrm{NaCl}): 2232 \mathrm{~cm}^{-1} ;{ }^{1} \mathrm{H}$ NMR (400

$\left.\mathrm{MHz}, \mathrm{CDCl}_{3}\right) \delta 7.53-7.48(\mathrm{~m}, 2 \mathrm{H}), 7.42-7.31(\mathrm{~m}, 3 \mathrm{H}), 3.57(\mathrm{~d}, J=4.0 \mathrm{~Hz}, 1 \mathrm{H}), 3.25(\mathrm{qd}, J=5.2$, $4.0 \mathrm{~Hz}, 1 \mathrm{H}), 1.49(\mathrm{~d}, J=5.2 \mathrm{~Hz}, 3 \mathrm{H}) ;{ }^{13} \mathrm{C} \mathrm{NMR}\left(101 \mathrm{MHz}, \mathrm{CDCl}_{3}\right) \delta 132.9,129.7,128.7,121.4$, 78.01, 77.96, 73.5, 70.1, 55.1, 46.0, 15.2. Anal. Calcd for $\mathrm{C}_{13} \mathrm{H}_{10} \mathrm{O}: \mathrm{C}, 85.69 ; \mathrm{H}, 5.53$. Found: C, 85.44; H, 5.45 . 
Table 1, entry 16

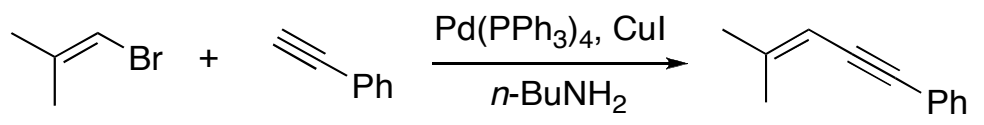

The enyne was prepared via Sonogashira coupling of 1-bromo-2-methylpropene and phenylacetylene (79\%): Colorless oil; IR (NaCl): 2197, $1489 \mathrm{~cm}^{-1}$; ${ }^{1} \mathrm{H}$ NMR (400 MHz, $\left.\mathrm{CDCl}_{3}\right) \delta$ 7.45-7.40 (m, 2H), 7.33-7.24 (m, 3H), $5.48(\mathrm{~s}, 1 \mathrm{H}), 1.98(\mathrm{~s}, 3 \mathrm{H}), 1.87(\mathrm{~s}, 3 \mathrm{H}) ;{ }^{13} \mathrm{C}$ NMR (101 MHz, $\left.\mathrm{CDCl}_{3}\right) \delta 149.2,131.5,128.5,127.9,124.2,105.4,91.6,87.9,25.2,21.4$.

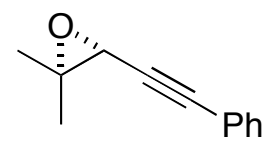

Colorless oil; $[\alpha]_{\mathrm{D}}^{25}=+3.56\left(c 0.59, \mathrm{CHCl}_{3}\right)(94 \%$ ee $) ;$ IR $(\mathrm{NaCl}): 2221,1490 \mathrm{~cm}^{-1} ;{ }^{1} \mathrm{H}$ NMR $\left(400 \mathrm{MHz}, \mathrm{CDCl}_{3}\right) \delta$ 7.47-7.43 (m, 2H), 7.36-7.28 (m, 3H), $3.44(\mathrm{~s}, 1 \mathrm{H}), 1.52(\mathrm{~s}, 3 \mathrm{H}), 1.41$ (s, $3 \mathrm{H}) ;{ }^{13} \mathrm{C} \mathrm{NMR}\left(101 \mathrm{MHz}, \mathrm{CDCl}_{3}\right) \delta 132.1,128.9,128.5,122.5,85.5,85.2,61.2,52.5,23.7$, 20.6. Anal. Calcd for $\mathrm{C}_{12} \mathrm{H}_{13} \mathrm{O}$ : C, 83.69; H, 7.02. Found: C, 83.80; H, 7.00.

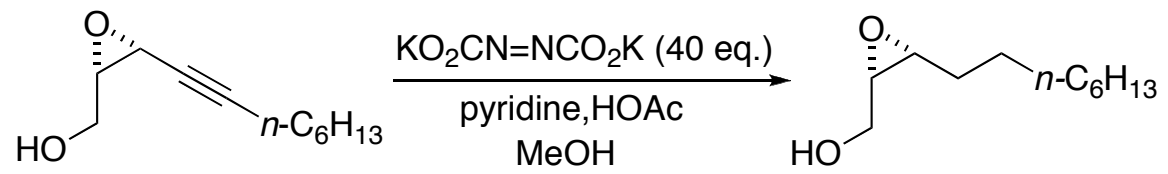

(2S,3R)-2,3-epoxyundecan-1-ol: To a solution of the propargyl epoxide (0.096 g, $0.53 \mathrm{mmol})$ (96\% ee) in $\mathrm{MeOH}(4 \mathrm{~mL})$ and pyridine $(0.86 \mathrm{~mL})$ at $\mathrm{rt}$ was added potassium azodicarboxylate $(1.03 \mathrm{~g}, 5.3 \mathrm{mmol})$ at the beginning. The same amount of potassium azodicarboxylate $(1.03 \mathrm{~g}$, $5.3 \mathrm{mmol})$ was subsequently added at the 12th, 24th, and 36th hour. Acetic acid $(2.57 \mathrm{~mL}, 44.52$ $\mathrm{mmol}$, in $5 \mathrm{~mL} \mathrm{MeOH}$ ) was constantly added over $60 \mathrm{~h}$ via syringe pump. The reaction was then diluted with $\mathrm{Et}_{2} \mathrm{O}$ and washed with water until no more gas bubbles evolved. The aqueous phase 
was then extracted with $\mathrm{Et}_{2} \mathrm{O}$ and the combined organic phases were washed with sat. aq. $\mathrm{CuSO}_{4}$, $\mathrm{H}_{2} \mathrm{O}$, brine, dried $\left(\mathrm{Na}_{2} \mathrm{SO}_{4}\right)$, filtered, and concentrated. The residue was purified by column (1:1 pet. ether- $\left.\mathrm{Et}_{2} \mathrm{O}\right)$ to yield the saturated epoxide as a white solid $(0.05 \mathrm{~g}, 53 \%):[\alpha]_{\mathrm{D}}{ }^{25}=-2.7(c$ $\left.0.37, \mathrm{CHCl}_{3}\right)$; lit. $[\alpha]_{\mathrm{D}}{ }^{25}=-3.5\left(c 1.3, \mathrm{CHCl}_{3}\right)(>80 \% \text { ee })^{*} ; \quad \mathrm{IR}(\mathrm{NaCl}): 3297,1469 \mathrm{~cm}^{-1} ;{ }^{1} \mathrm{H}$ NMR (400 MHz, $\left.\mathrm{CDCl}_{3}\right) \delta$ 3.92-3.82 (m, 1H), 3.73-3.64 (m, 1H), 3.16 (dt, J=7.2, 4.0 Hz, 1H), 3.08-3.02 (m, 1H), $1.66(\mathrm{~s}, 1 \mathrm{H}), 1.62-1.22(\mathrm{~m}, 14 \mathrm{H}), 0.89(\mathrm{t}, J=6.8 \mathrm{~Hz}, 1 \mathrm{H}) ;{ }^{13} \mathrm{C}$ NMR $(101$ $\left.\mathrm{MHz}, \mathrm{CDCl}_{3}\right) \delta 61.2,57.6,57.0,32.1,29.7,29.6,29.4,28.2,26.9,22.9,14.3$.

* Gao, Y.; Klunder, J.M.; Hanson, R.M.; Masamune, H.; Ko, S.Y.; Sharpless, K.B. J. Am. Chem. Soc. 1987, 109, 5765. 
The chromatograms for the determination of enantiomeric excess of the epoxides from the asymmetric epoxidation of conjugated enynes catalyzed by ketone 2 .

Table 1, Entry 1

$\overbrace{p h}$

HPLC Cond.: Column: Chiralcel OD (Column No. OD00CE-DL010), Chiral Technologies, Inc.

Eluent: Hexanes/IPA (96/4); Flow Rate: 1.0mL/min; Detection: UV $254 \mathrm{~nm}$
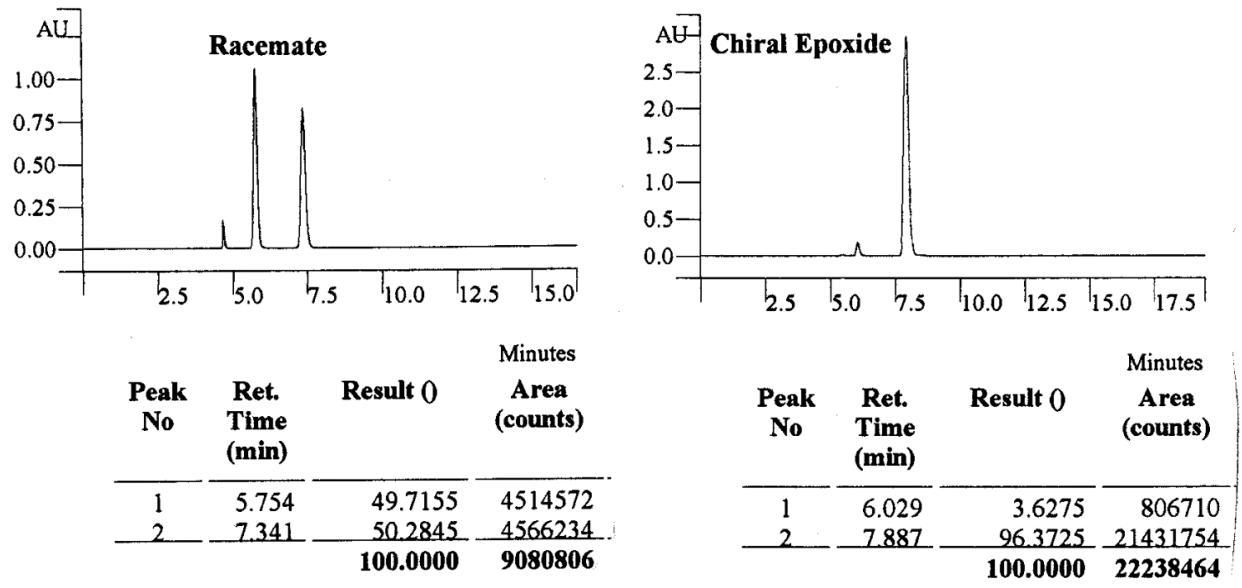

Table 1, Entry 2

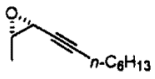

GC Cond.: Column: Chiraldex B-DM (Cat. No. 77023), Adv. Separation Technologies, Inc.

Oven: $100^{\circ} \mathrm{C}$; Carrier: Helium, head pressure $20 \mathrm{psi}$; Detection: FID $250^{\circ} \mathrm{C}$

Racemate

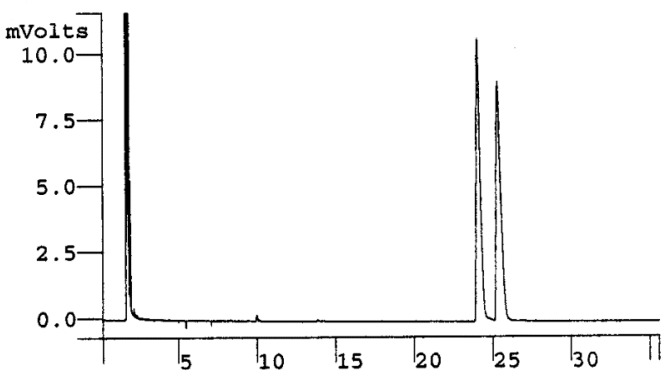

\begin{tabular}{ccccc}
$\begin{array}{c}\text { Peak } \\
\text { No }\end{array}$ & $\begin{array}{c}\text { Peak } \\
\text { Name }\end{array}$ & $\begin{array}{c}\text { Result } \\
0\end{array}$ & $\begin{array}{c}\text { Ret. } \\
\text { Time } \\
(\mathbf{m i n})\end{array}$ & $\begin{array}{c}\text { Minutes } \\
\text { Area } \\
\text { (counts) }\end{array}$ \\
\hline 1 \\
\hline 2
\end{tabular}

Chiral Epoxide

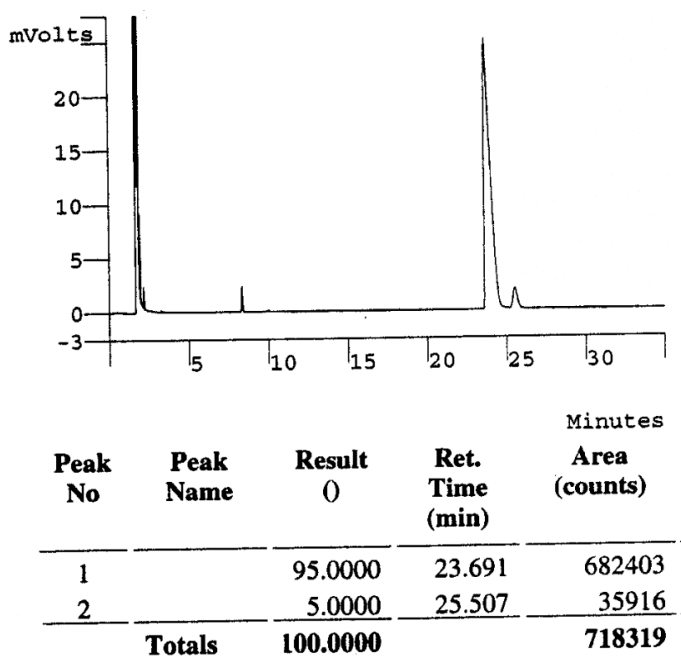


Table 1, Entry 3

NoBn

GC Cond.: Column: Chiraldex B-DM (Cat. No. 77023), Adv. Separation Technologies, Inc.
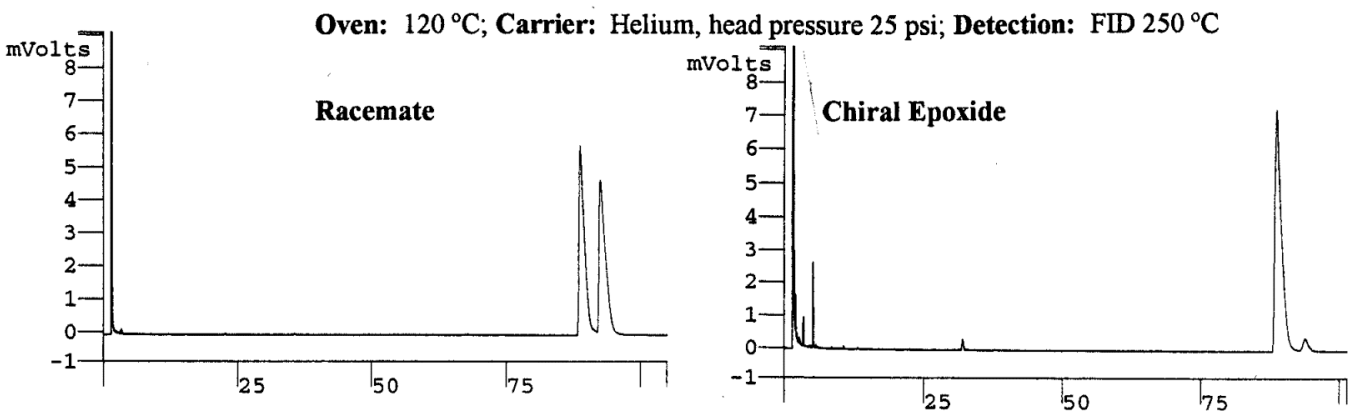

\begin{tabular}{cccccccccc}
$\begin{array}{c}\text { Peak } \\
\text { No }\end{array}$ & $\begin{array}{c}\text { Peak } \\
\text { Name }\end{array}$ & $\begin{array}{c}\text { Result } \\
0\end{array}$ & $\begin{array}{c}\text { Ret. } \\
\text { Time } \\
(\mathbf{m i n})\end{array}$ & $\begin{array}{c}\text { Minutes } \\
\text { Area } \\
\text { (counts) }\end{array}$ & $\begin{array}{c}\text { Peak } \\
\text { No }\end{array}$ & $\begin{array}{c}\text { Peak } \\
\text { Name }\end{array}$ & $\begin{array}{c}\text { Result } \\
0\end{array}$ & $\begin{array}{c}\text { Ret. } \\
\text { Time } \\
\text { (min) }\end{array}$ & $\begin{array}{c}\text { Minutes } \\
\text { Area } \\
\text { (counts) }\end{array}$ \\
\hline 1 \\
\hline
\end{tabular}

Table 1, Entry 4<smiles>CCC#CCCCO</smiles>

HPLC Cond.: Column: Chirapak AD (Column No. AD00CE-EA001), Chiral Technologies, Inc.

Eluent: Hexanes/IPA (92/8); Flow Rate: $1.0 \mathrm{~mL} / \mathrm{min}$; Detection: UV $270 \mathrm{~nm}$

Racemate

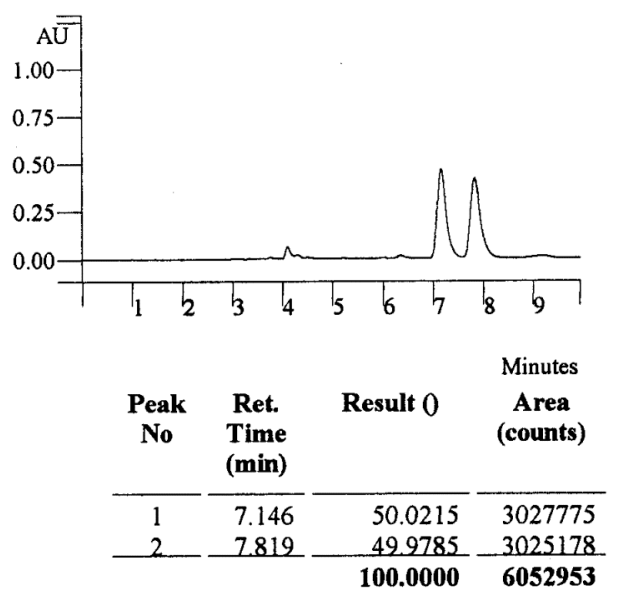

Chiral Epoxide

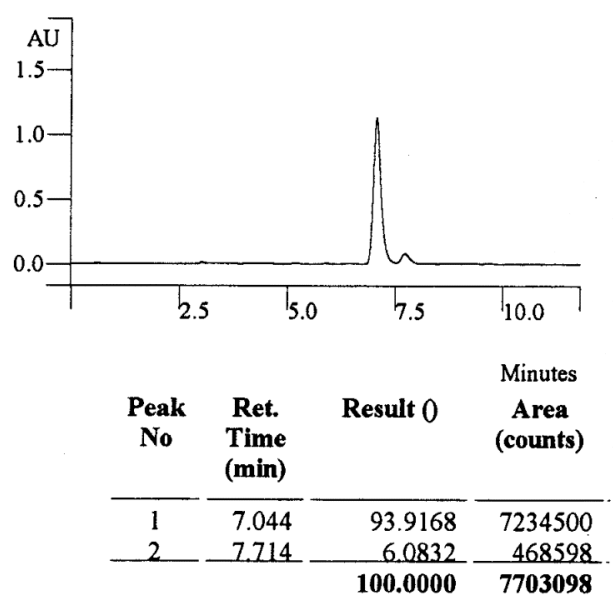


Table 1, Entry 5<smiles>CCCCC1CC#CC1</smiles>

GC Cond.: Column: Chiraldex B-DM (Cat. No. 77023), Adv. Separation Technologies, Inc.

Oven: $135^{\circ} \mathrm{C}$; Carrier: Helium, head pressure 25 psi; Detection: FID $250^{\circ} \mathrm{C}$
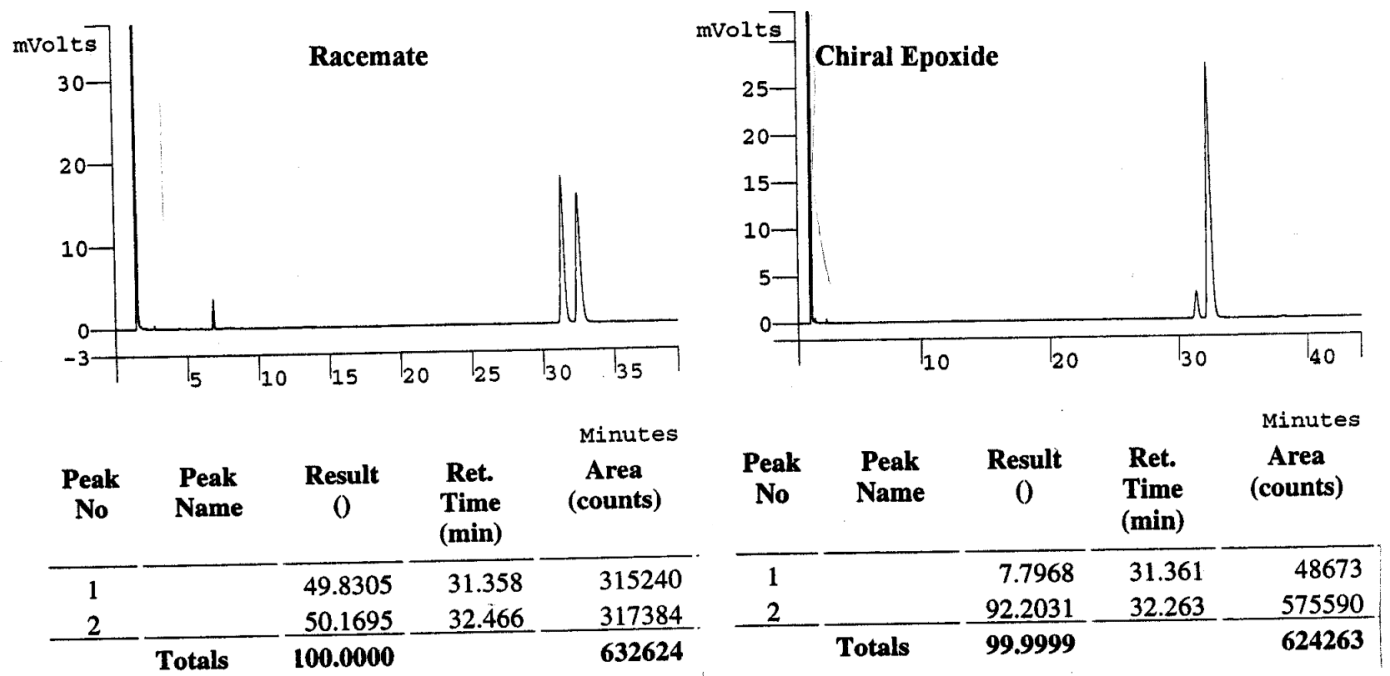

Table 1, Entry 6<smiles>[NH3+]C#CC(=O)CO</smiles>

GC Cond.: Column: Chiraldex B-DM (Cat. No. 77023), Adv. Separation Technologies, Inc.

Oven: $110^{\circ} \mathrm{C}$; Carrier: Helium, head pressure 25 psi; Detection: FID $250^{\circ} \mathrm{C}$

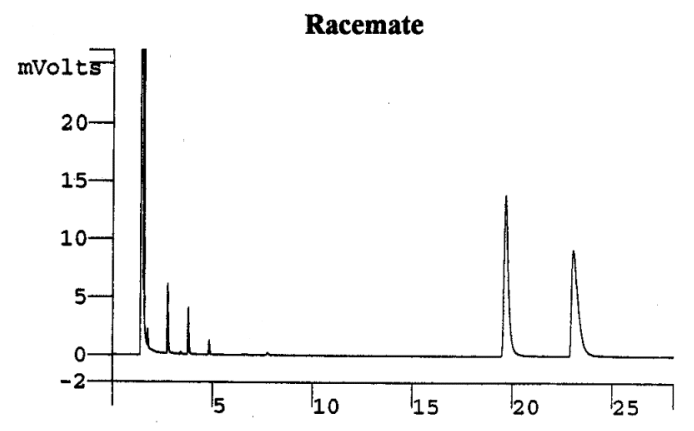

Chiral Epoxide

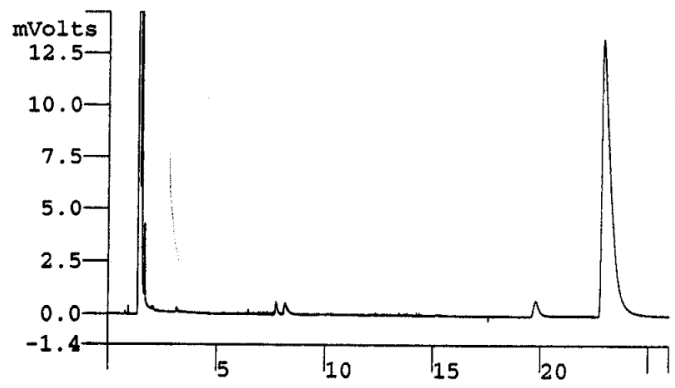

\begin{tabular}{|c|c|c|c|c|}
\hline $\begin{array}{c}\text { Peak } \\
\text { No }\end{array}$ & $\begin{array}{c}\text { Peak } \\
\text { Name }\end{array}$ & $\begin{array}{c}\text { Result } \\
0\end{array}$ & $\begin{array}{l}\text { Ret. } \\
\text { Time } \\
\text { (min) }\end{array}$ & $\begin{array}{l}\text { Minutes } \\
\text { Area } \\
\text { (counts) }\end{array}$ \\
\hline 1 & & 50.0373 & 19.636 & 209856 \\
\hline 2 & & 49.9627 & 23.036 & 209543 \\
\hline & Totals & 100.0000 & & 419399 \\
\hline
\end{tabular}

\begin{tabular}{ccccc}
$\begin{array}{c}\text { Peak } \\
\text { No }\end{array}$ & $\begin{array}{c}\text { Peak } \\
\text { Name }\end{array}$ & $\begin{array}{c}\text { Result } \\
0\end{array}$ & $\begin{array}{c}\text { Ret. } \\
\text { Time } \\
(\mathbf{m i n})\end{array}$ & $\begin{array}{c}\text { Minutes } \\
\text { Area } \\
\text { (counts) }\end{array}$ \\
\hline 1 \\
\hline 2
\end{tabular}


Table 1, Entry 7<smiles>[N+]=CC=CC(=O)CCCO</smiles>

GC Cond.: Column: Chiraldex B-DM (Cat. No. 77023), Adv. Separation Technologies, Inc.

Oven: $130^{\circ} \mathrm{C}$; Carrier: Helium, head pressure 25 psi; Detection: FID $250^{\circ} \mathrm{C}$
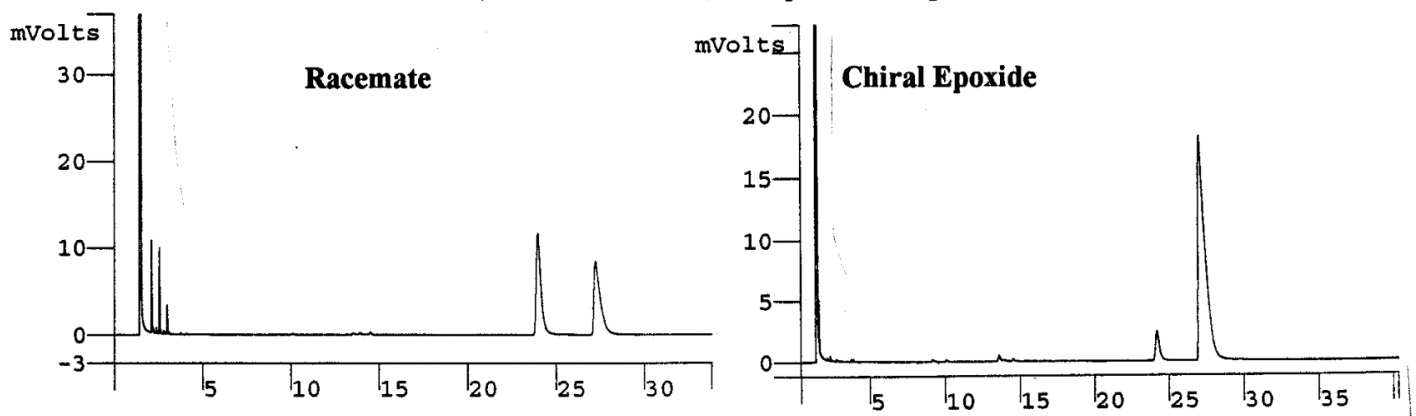

\begin{tabular}{cccccccccc}
$\begin{array}{c}\text { Peak } \\
\text { No }\end{array}$ & $\begin{array}{c}\text { Peak } \\
\text { Name }\end{array}$ & $\begin{array}{c}\text { Result } \\
0\end{array}$ & $\begin{array}{c}\text { Ret. } \\
\text { Time } \\
(\mathbf{m i n})\end{array}$ & $\begin{array}{c}\text { Minutes } \\
\text { (counts) }\end{array}$ & $\begin{array}{c}\text { Peak } \\
\text { No }\end{array}$ & $\begin{array}{c}\text { Peak } \\
\text { Name }\end{array}$ & $\begin{array}{c}\text { Result } \\
0\end{array}$ & $\begin{array}{c}\text { Ret. } \\
\text { Time } \\
(\mathbf{m i n})\end{array}$ & $\begin{array}{c}\text { Minutes } \\
\text { Area } \\
\text { (counts) }\end{array}$ \\
\hline 1 \\
\hline
\end{tabular}

Table 1, Entry 8<smiles>C#CC(=O)CCCCOC</smiles>

GC Cond.: Column: Chiraldex B-DM (Cat. No. 77023), Adv. Separation Technologies, Inc. Oven: $125^{\circ} \mathrm{C}$; Carrier: Helium, head pressure 25 psi; Detection: FID $250^{\circ} \mathrm{C}$
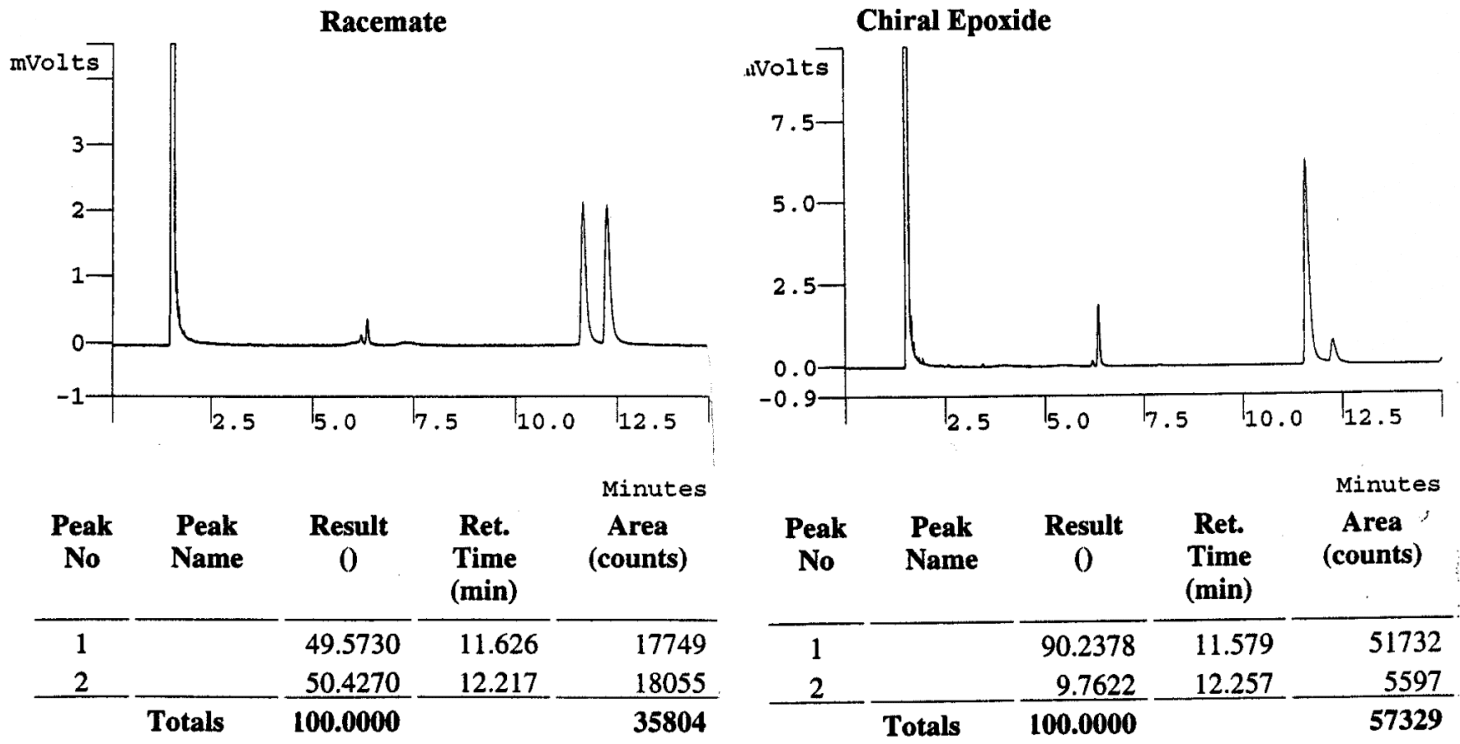
Table 1, Entry 9<smiles></smiles>

HPLC Cond.: Column: Chiralcel OD (Column No. OD00CE-DL010), Chiral Technologies, Inc.
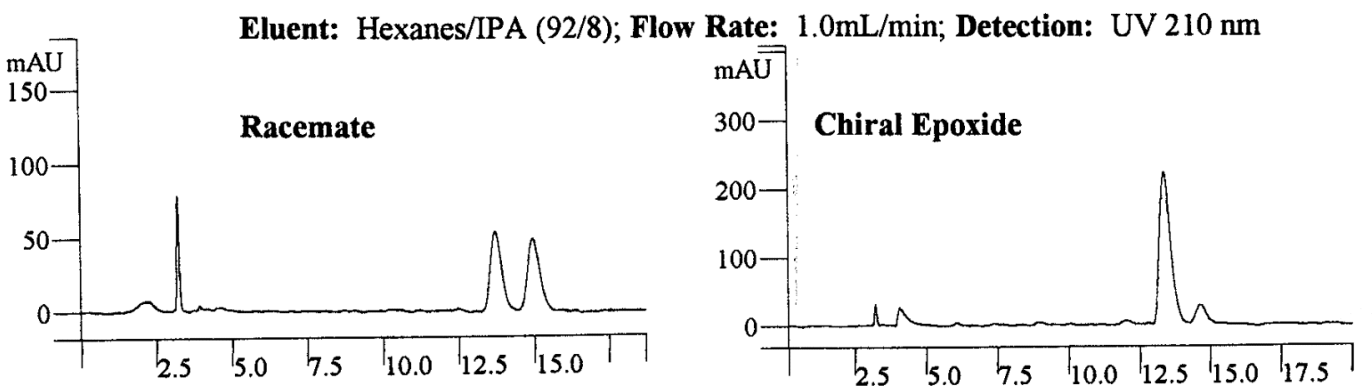

\begin{tabular}{|c|c|c|c|}
\hline $\begin{array}{c}\text { Peak } \\
\text { No }\end{array}$ & $\begin{array}{l}\text { Ret. } \\
\text { Time } \\
\text { (min) }\end{array}$ & Result 0 & $\begin{array}{c}\text { Minutes } \\
\text { Area } \\
\text { (counts) }\end{array}$ \\
\hline 1 & 13.686 & 50.2666 & 721994 \\
\hline 2 & 14.934 & 49.7334 & 714334 \\
\hline & & 100.0000 & 1436328 \\
\hline
\end{tabular}

\begin{tabular}{cccc}
$\begin{array}{c}\text { Peak } \\
\text { No }\end{array}$ & $\begin{array}{c}\text { Ret. } \\
\text { Time } \\
(\mathbf{m i n})\end{array}$ & Result 0 & $\begin{array}{c}\text { Minutes } \\
\text { Area } \\
\text { (counts) }\end{array}$ \\
\hline 1 & $\frac{13.339}{2}$ & $\frac{14.659}{90.0670}$ & $\frac{9.9330}{3212628}$ \\
\hline $\mathbf{1 0 0 . 0 0 0 0}$ & $\frac{354304}{\mathbf{3 5 6 6 9 3 2}}$
\end{tabular}

Table 1, Entry 10<smiles>CCCCC#CC=CCCCCO</smiles>

HPLC Cond.: Column: Chiralcel OJ (Column No. OJ00CE-DE008), Chiral Technologies, Inc. Eluent: Hexanes/IPA (92/8); Flow Rate: $1.0 \mathrm{~mL} / \mathrm{min}$; Detection: UV $210 \mathrm{~nm}$

Racemate

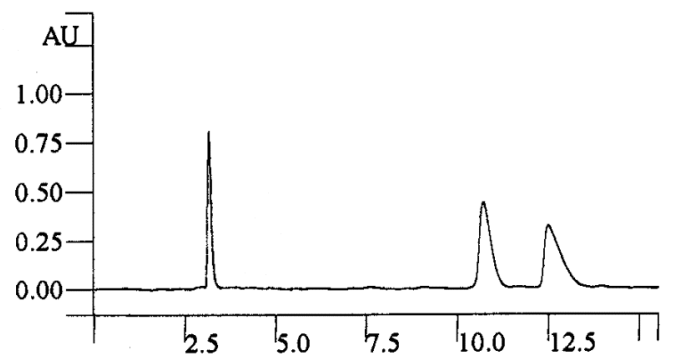

\begin{tabular}{|c|c|c|c|}
\hline $\begin{array}{c}\text { Peak } \\
\text { No }\end{array}$ & $\begin{array}{l}\text { Ret. } \\
\text { Time } \\
\text { (min) }\end{array}$ & Result 0 & $\begin{array}{c}\text { Minutes } \\
\text { Area } \\
\text { (counts) }\end{array}$ \\
\hline $\begin{array}{l}1 \\
2 \\
\end{array}$ & $\begin{array}{l}10.704 \\
12.493 \\
\end{array}$ & $\begin{array}{l}49.5158 \\
50.4842 \\
\end{array}$ & $\begin{array}{r}5191821 \\
5293368\end{array}$ \\
\hline & & 100.0000 & 10485189 \\
\hline
\end{tabular}

Chiral Epoxide

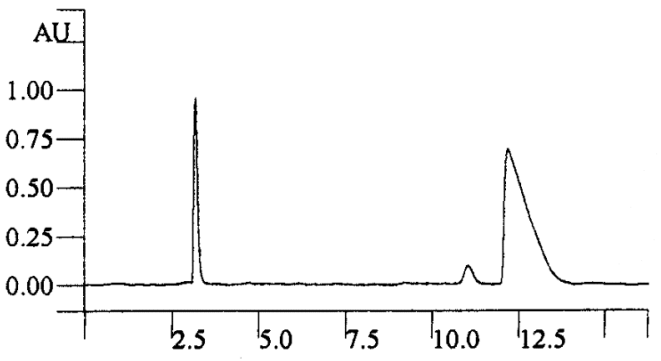

\begin{tabular}{cccc}
$\begin{array}{c}\text { Peak } \\
\text { No }\end{array}$ & $\begin{array}{c}\text { Ret. } \\
\text { Time } \\
\text { (min) }\end{array}$ & Result 0 & $\begin{array}{c}\text { Minutes } \\
\text { Area } \\
\text { (counts) }\end{array}$ \\
\hline 1 & $\frac{11.014}{12.161} \frac{4.8570}{\frac{95.1430}{\mathbf{1 0 0 . 0 0 0 0}}} \frac{17102756}{\mathbf{1 7 9 7 5 8 3 6}}$.
\end{tabular}


Table 1, Entry 11

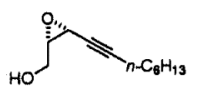

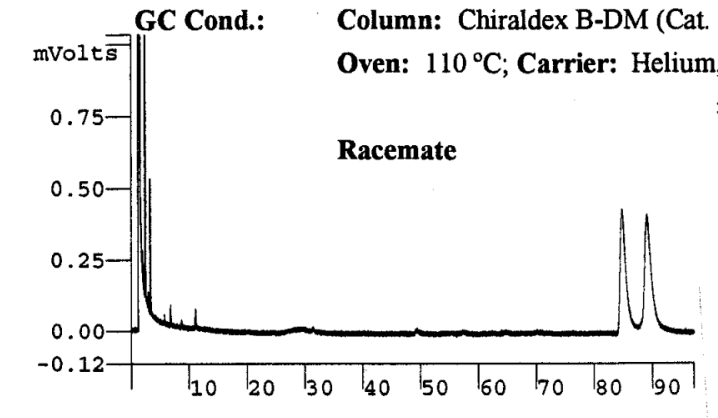

at. No. 77023), Adv. Separation Technologies, Inc
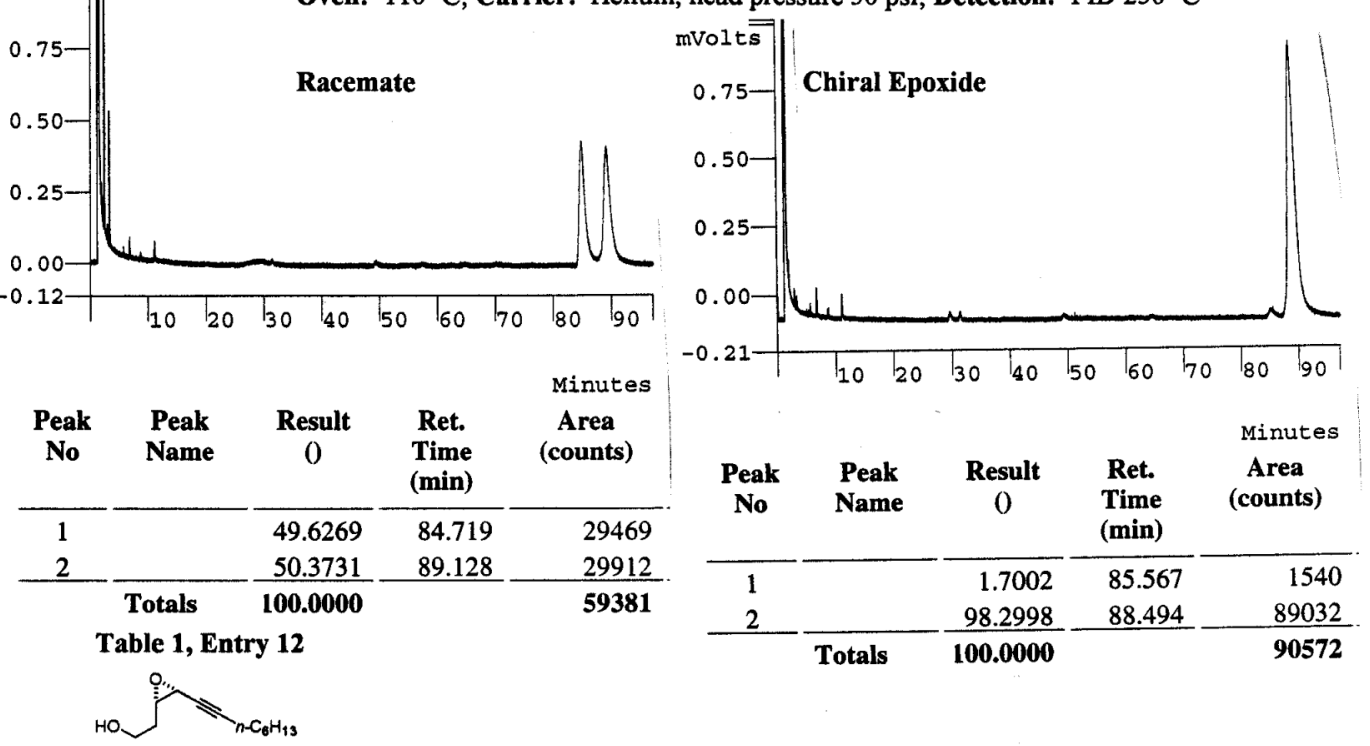

GC Cond.: Column: Chiraldex B-DM (Cat. No. 77023), Adv. Separation Technologies, Inc. Oven: $125^{\circ} \mathrm{C}$; Carrier: Helium, head pressure $25 \mathrm{psi}$; Detection: FID $250^{\circ} \mathrm{C}$

Racemate

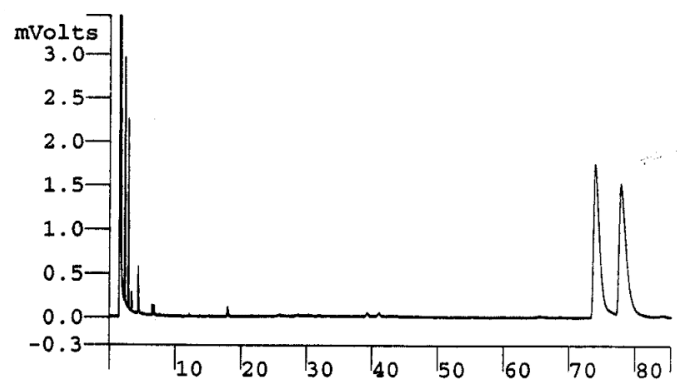

Chiral Epoxide

\begin{tabular}{cccc}
$\begin{array}{c}\text { Peak } \\
\text { No }\end{array}$ & $\begin{array}{c}\text { Peak } \\
\text { Name }\end{array}$ & $\begin{array}{c}\text { Result } \\
0\end{array}$ & $\begin{array}{c}\text { Ret. } \\
\text { Time } \\
(\mathbf{m i n})\end{array}$ \\
\hline 1 \\
\hline 2
\end{tabular}

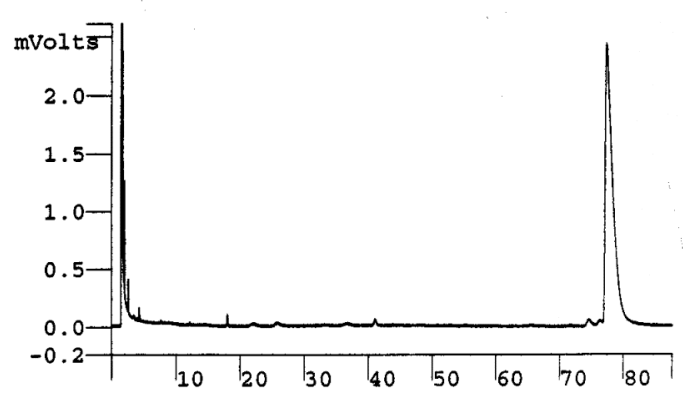

\begin{tabular}{ccccc}
$\begin{array}{c}\text { Peak } \\
\text { No }\end{array}$ & $\begin{array}{c}\text { Peak } \\
\text { Name }\end{array}$ & $\begin{array}{c}\text { Result } \\
0\end{array}$ & $\begin{array}{c}\text { Ret. } \\
\text { Time } \\
\text { (min) }\end{array}$ & $\begin{array}{c}\text { Minutes } \\
\text { Area } \\
\text { (counts) }\end{array}$ \\
\hline 1 & $\frac{1.4356}{2}$ & $\frac{74.537}{98.5644}$ & $\frac{77.316}{100.0000}$ & $\frac{195542}{1954}$ \\
\hline
\end{tabular}


Table 1, Entry 13

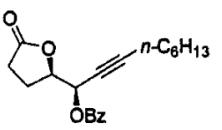

HPLC Cond.: Column: Chiralcel OD (Column No. OD00CE-DL010), Chiral Technologies, Inc.

Eluent: Hexanes/IPA (85/15); Flow Rate: $1.0 \mathrm{~mL} / \mathrm{min}$; Detection: UV $240 \mathrm{~nm}$
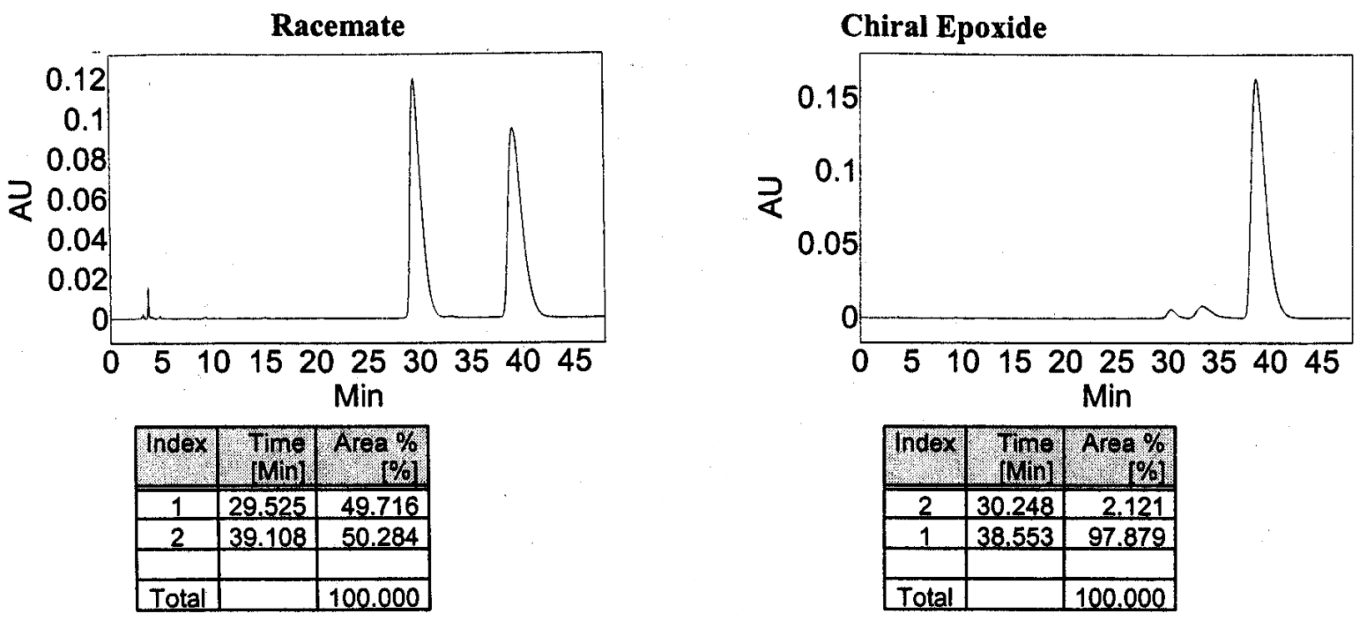

Table 1, Entry 14<smiles>O=C1OCCC#CCOC(=O)c2ccccc2O1</smiles>

HPLC Cond.: Column: Chiralcel OD (Column No. OD00CE-DL010), Chiral Technologies, Inc. Eluent: Hexanes/IPA (90/10); Flow Rate: $1.0 \mathrm{~mL} / \mathrm{min}$; Detection: UV $270 \mathrm{~nm}$
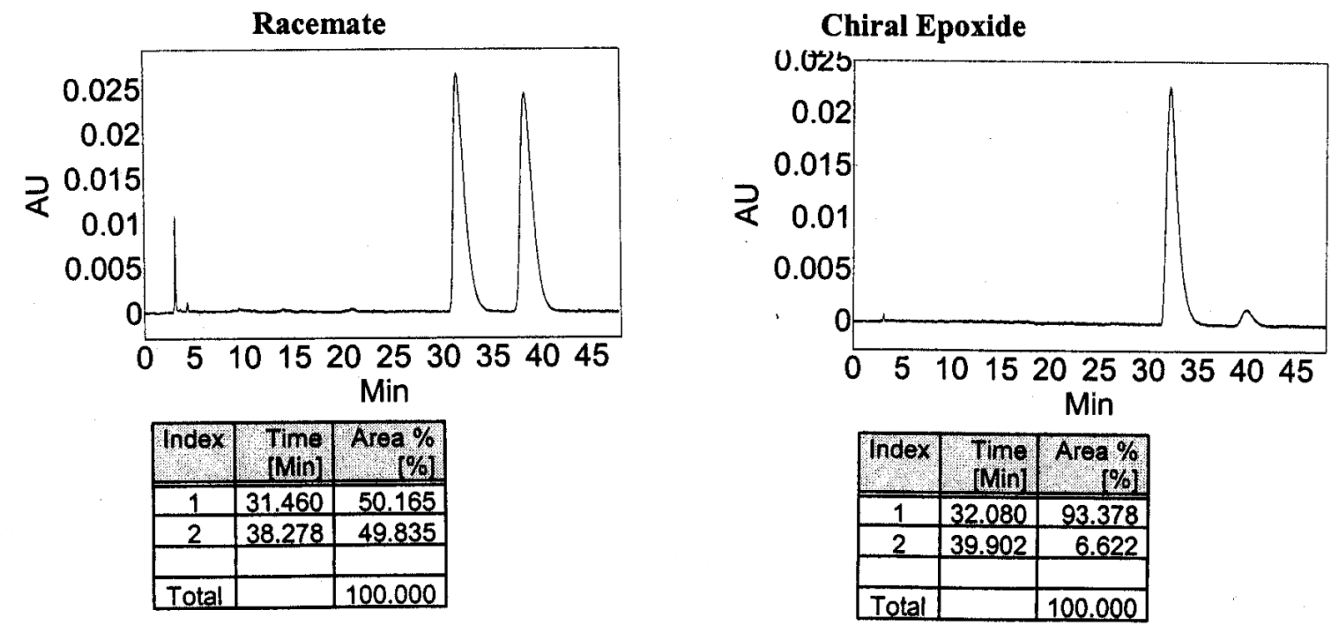
Table 1, Entry 15

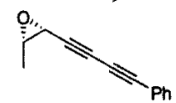

HPLC Cond.: Column: Chiralcel OD (Column No. OD00CE-DL010), Chiral Technologies, Inc.

Eluent: Hexanes/IPA (97/03); Flow Rate: $1.0 \mathrm{~mL} / \mathrm{min}$; Detection: UV $254 \mathrm{~nm}$
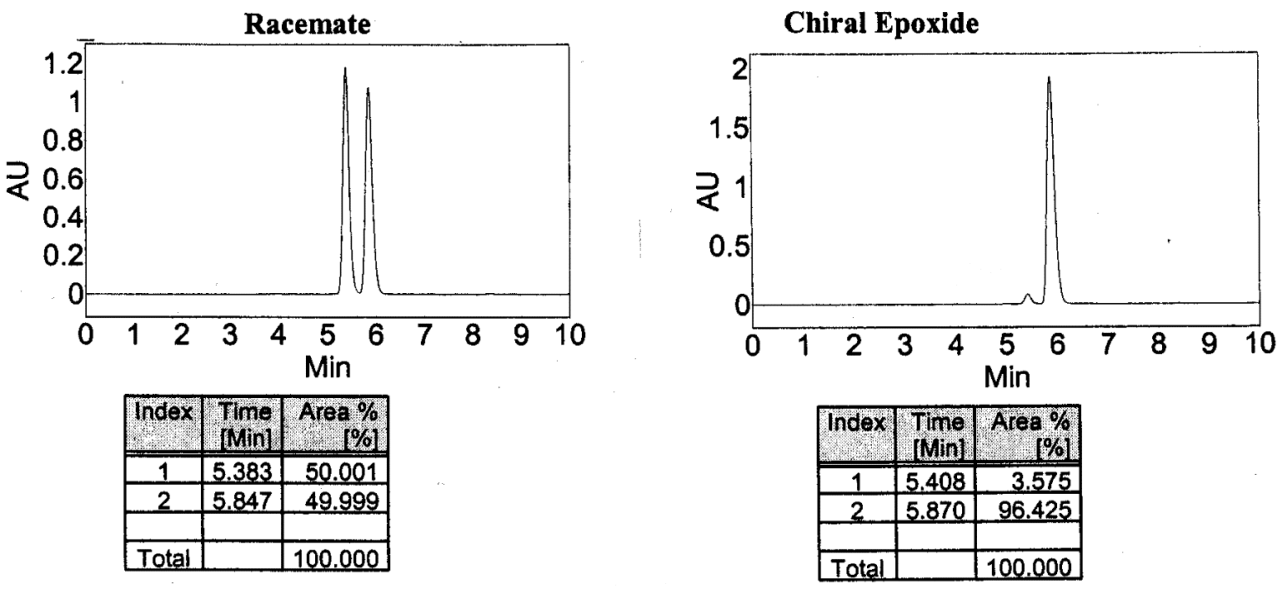

Table 1, Entry 16<smiles>CC(C)=CC#CP</smiles>

GC Cond.: Column: Chiraldex B-DM (Cat. No. 77023), Adv. Separation Technologies, Inc. Oven: $110^{\circ} \mathrm{C}$; Carrier: Helium, head pressure 20 psi; Detection: FID $250^{\circ} \mathrm{C}$
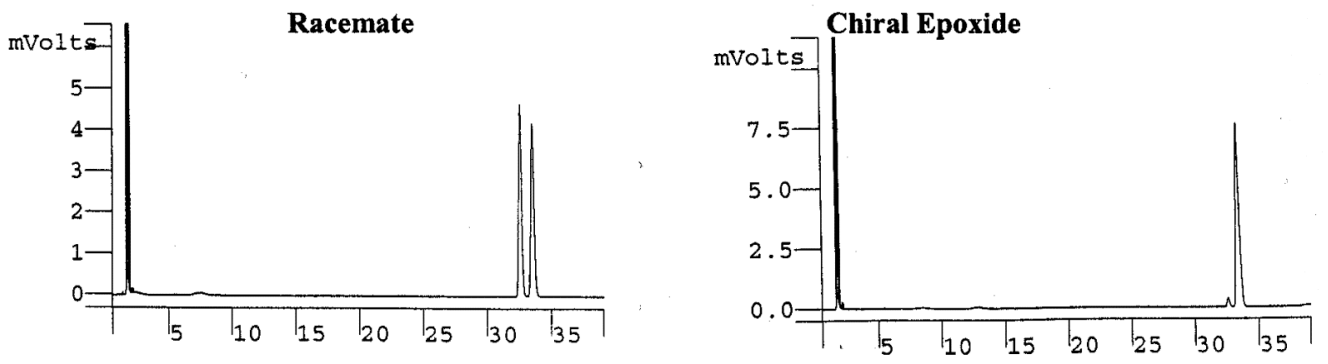

\begin{tabular}{ccccc}
$\begin{array}{c}\text { Peak } \\
\text { No }\end{array}$ & $\begin{array}{c}\text { Peak } \\
\text { Name }\end{array}$ & $\begin{array}{c}\text { Result } \\
0\end{array}$ & $\begin{array}{c}\text { Ret. } \\
\text { Time } \\
(\mathbf{m i n})\end{array}$ & $\begin{array}{c}\text { Minutes } \\
\text { (counts) }\end{array}$ \\
\hline 1 & & $\begin{array}{c}49.8989 \\
2\end{array}$ & $\frac{32.466}{50.1011}$ & $\frac{33.426}{71182}$ \\
\hline Totals & $\frac{71470}{\mathbf{1 0 0 . 0 0 0 0}}$ & &
\end{tabular}

\begin{tabular}{ccccr}
$\begin{array}{c}\text { Peak } \\
\text { No }\end{array}$ & $\begin{array}{c}\text { Peak } \\
\text { Name }\end{array}$ & $\begin{array}{c}\text { Result } \\
0\end{array}$ & $\begin{array}{c}\text { Ret. } \\
\text { Time } \\
(\mathbf{m i n})\end{array}$ & $\begin{array}{c}\text { Minutes } \\
\text { (counts) }\end{array}$ \\
\hline 1 & & 3.0543 & 32.607 & $\begin{array}{r}4579 \\
2\end{array}$ \\
\hline Totals & $\frac{96.9457}{\mathbf{1 0 0 . 0 0 0 0}}$ & $\frac{33.274}{145330}$ & & \\
$\mathbf{1 4 9 9 0 9}$
\end{tabular}




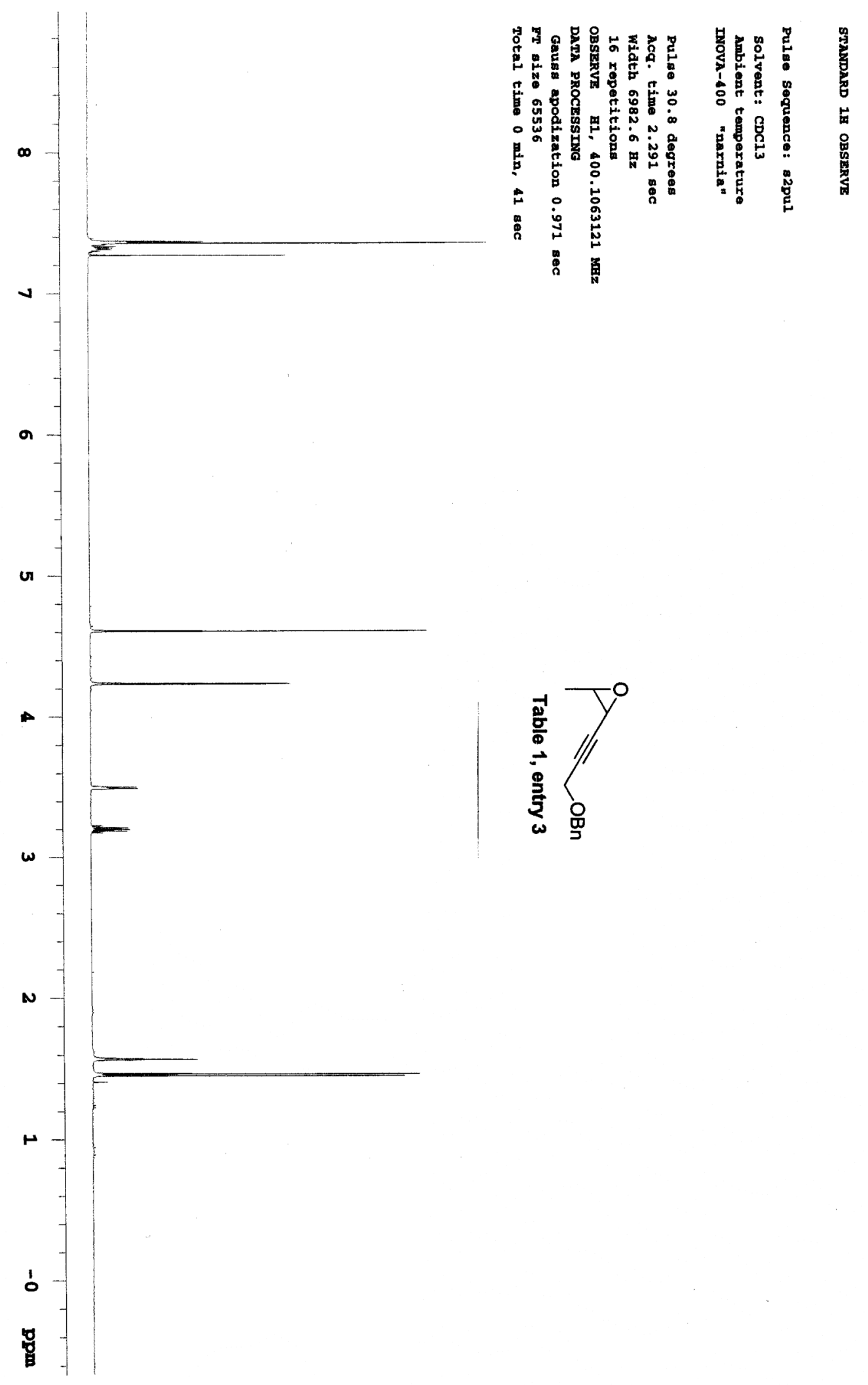




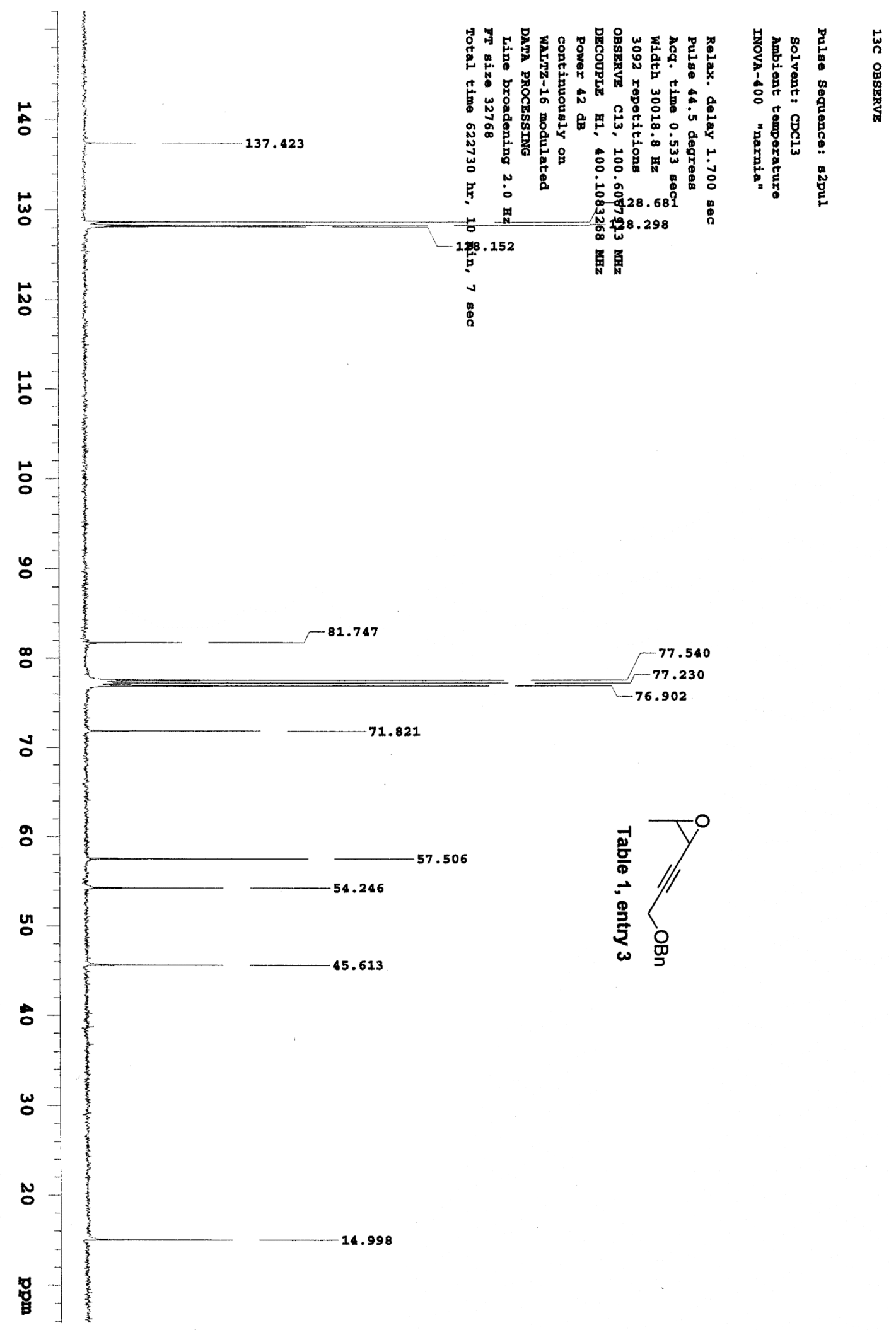




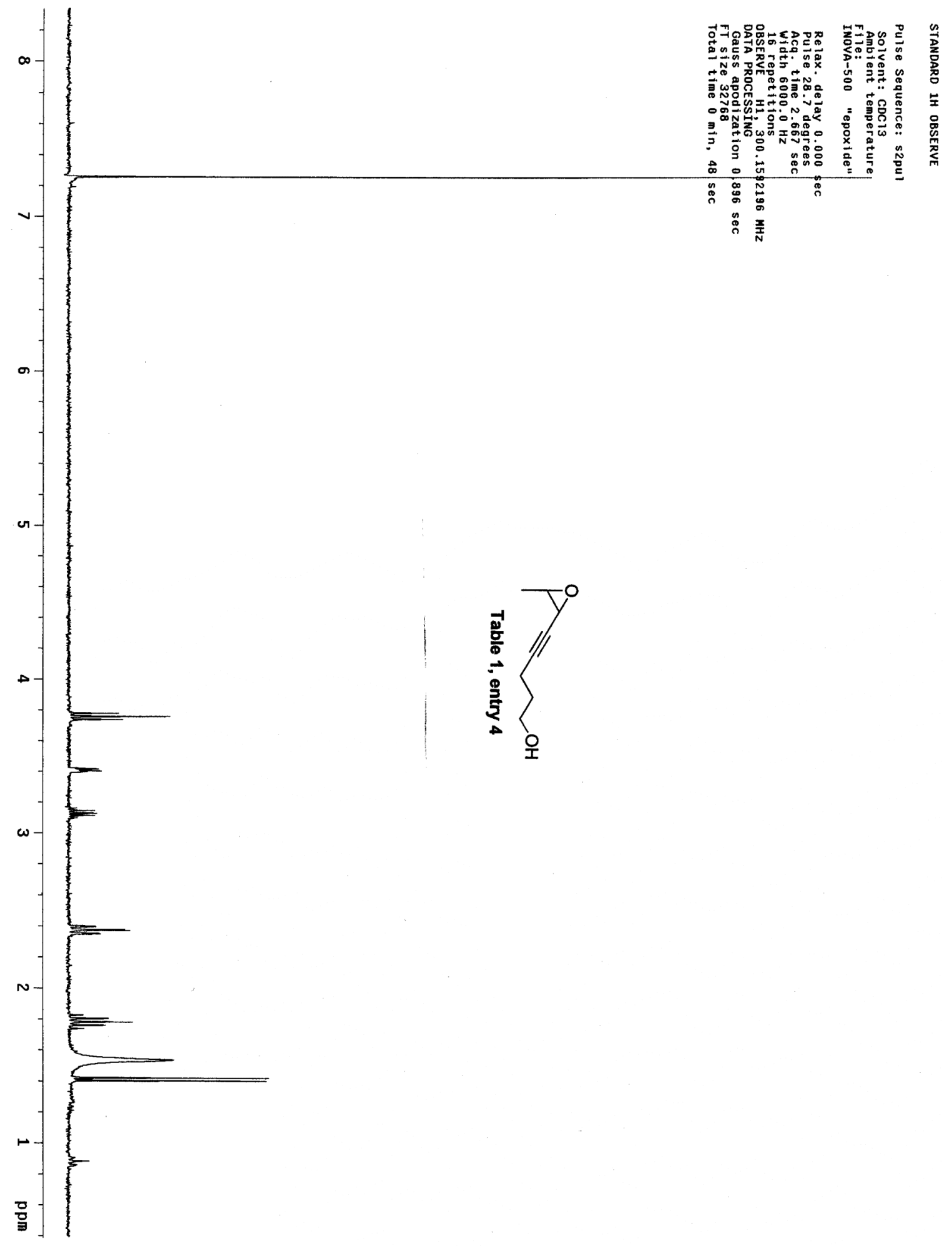




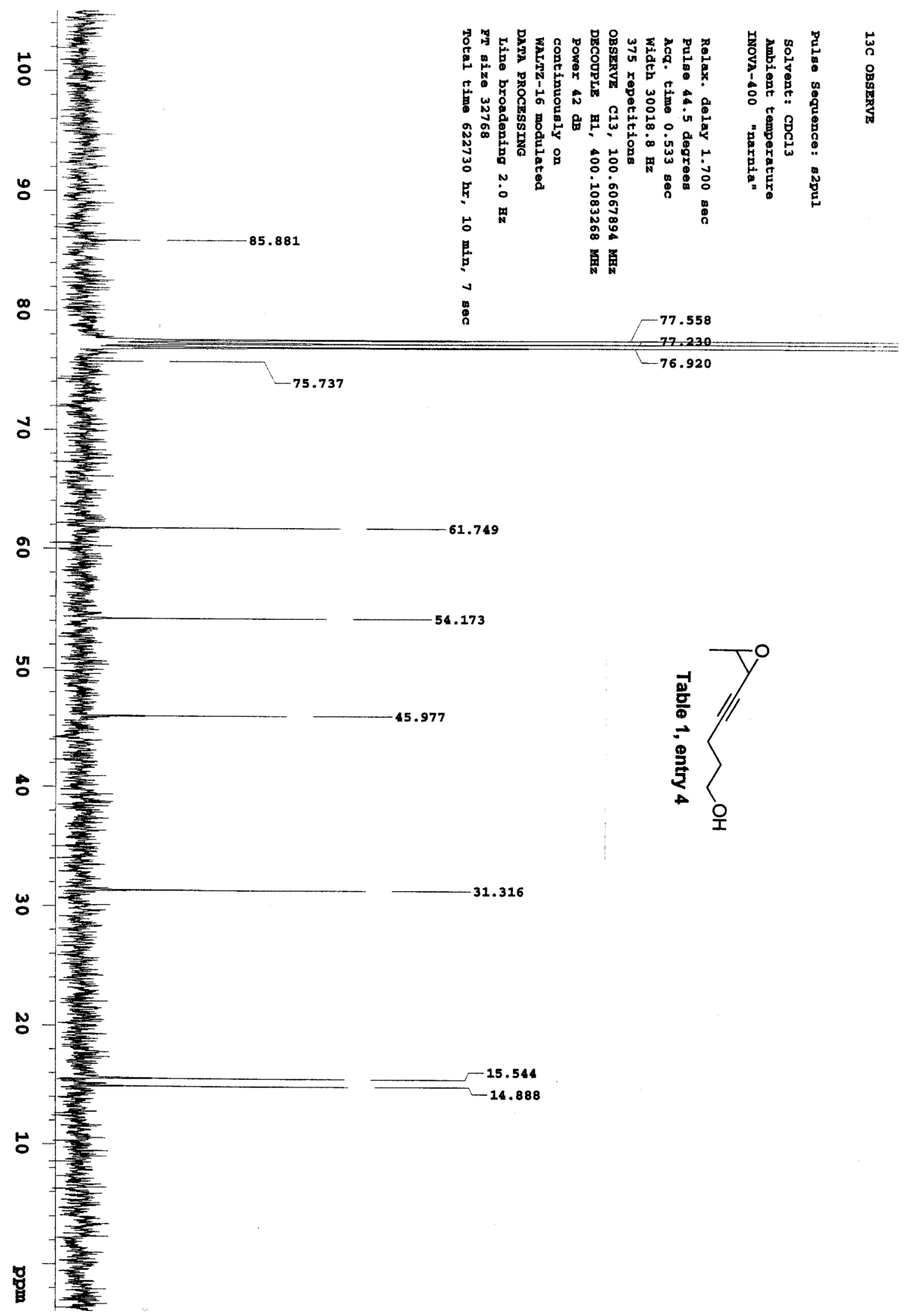




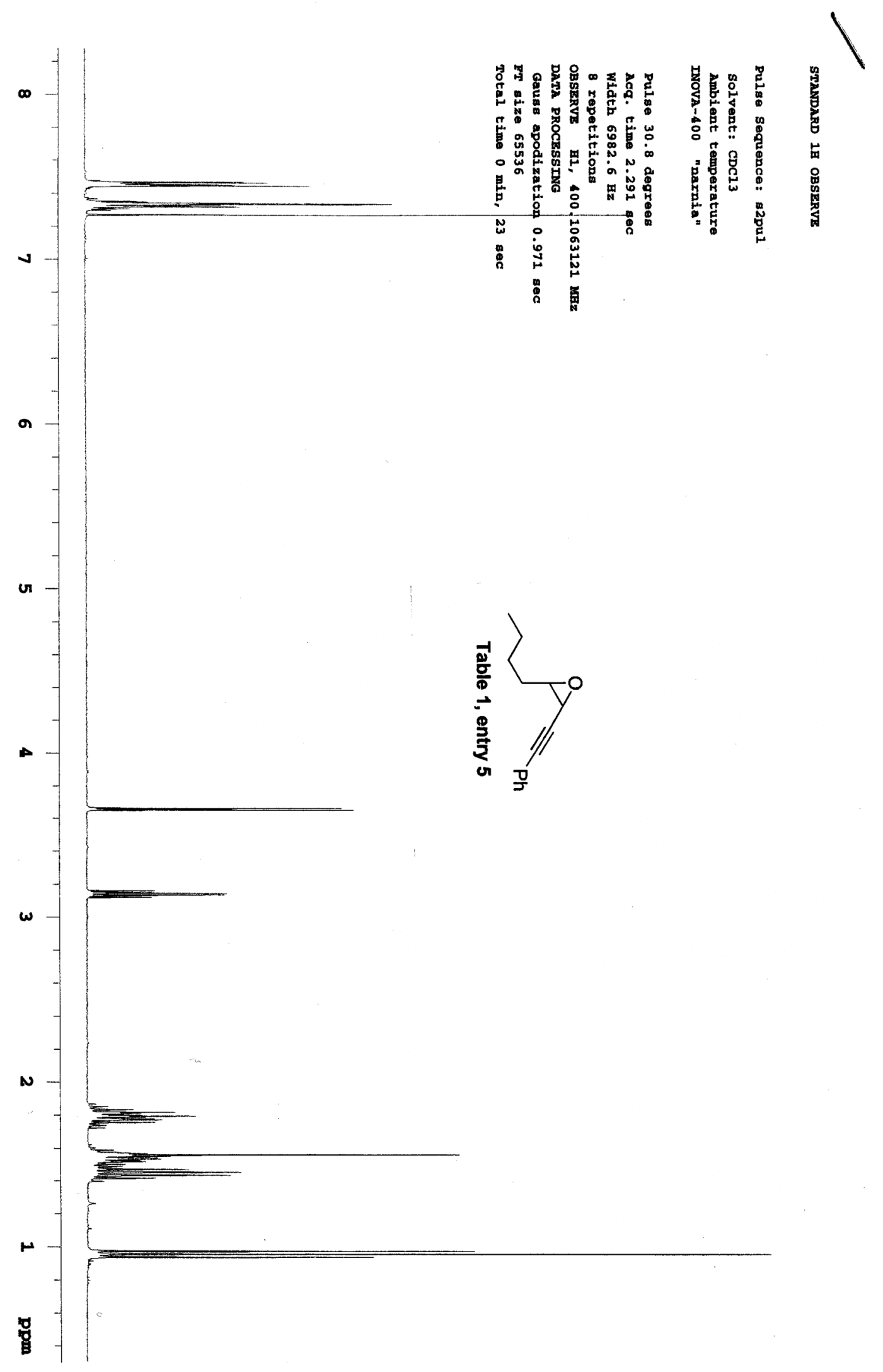




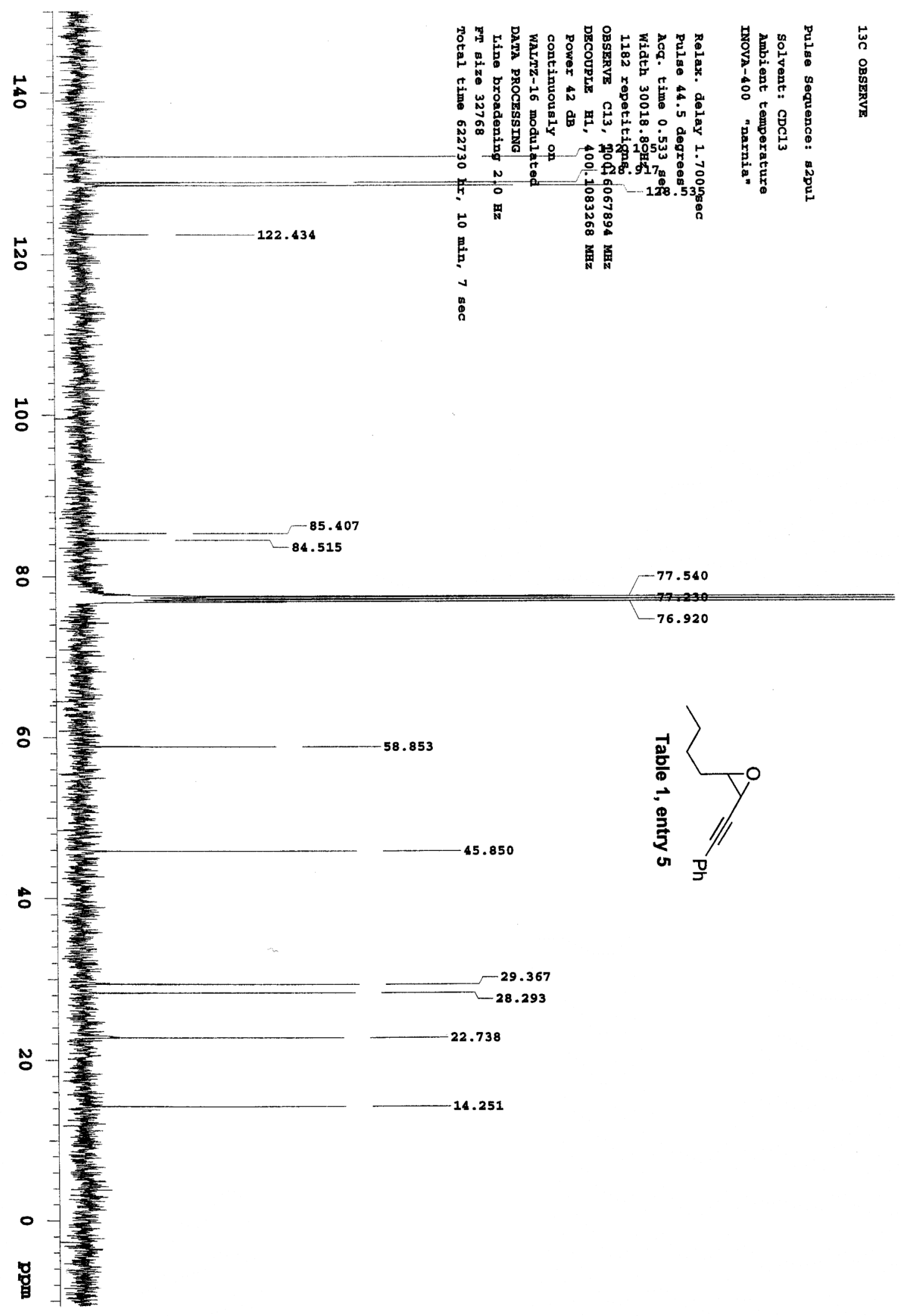




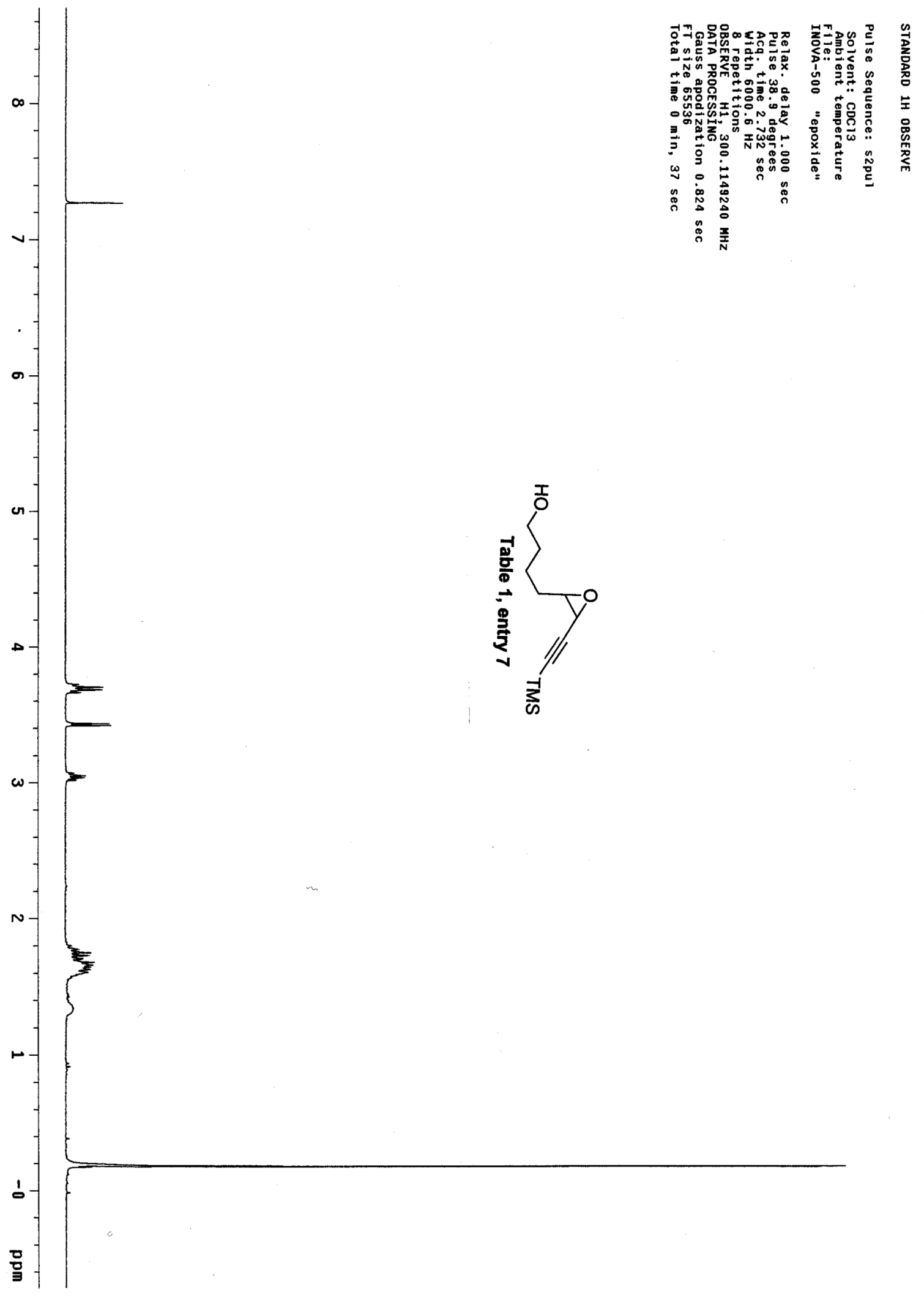



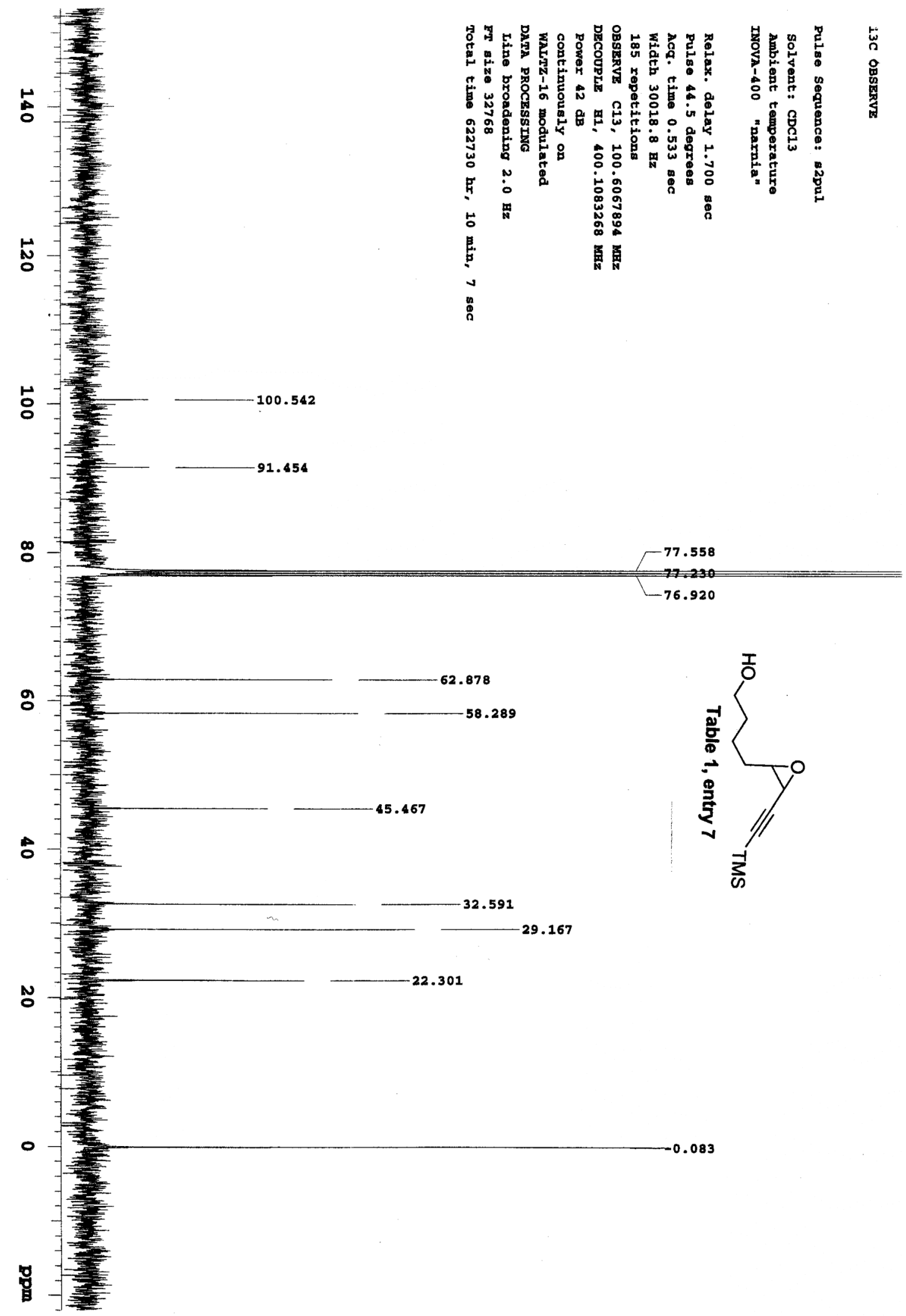


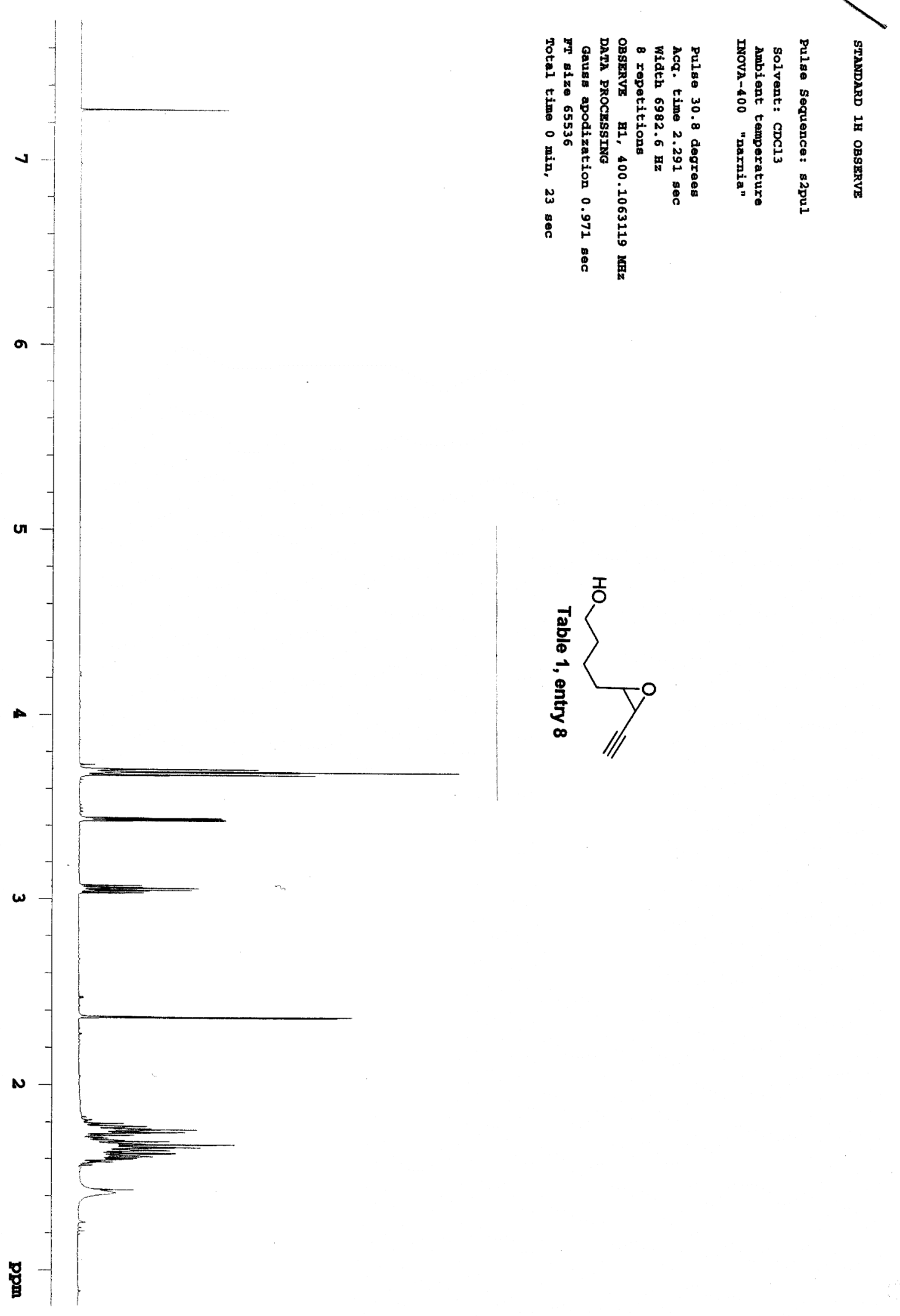




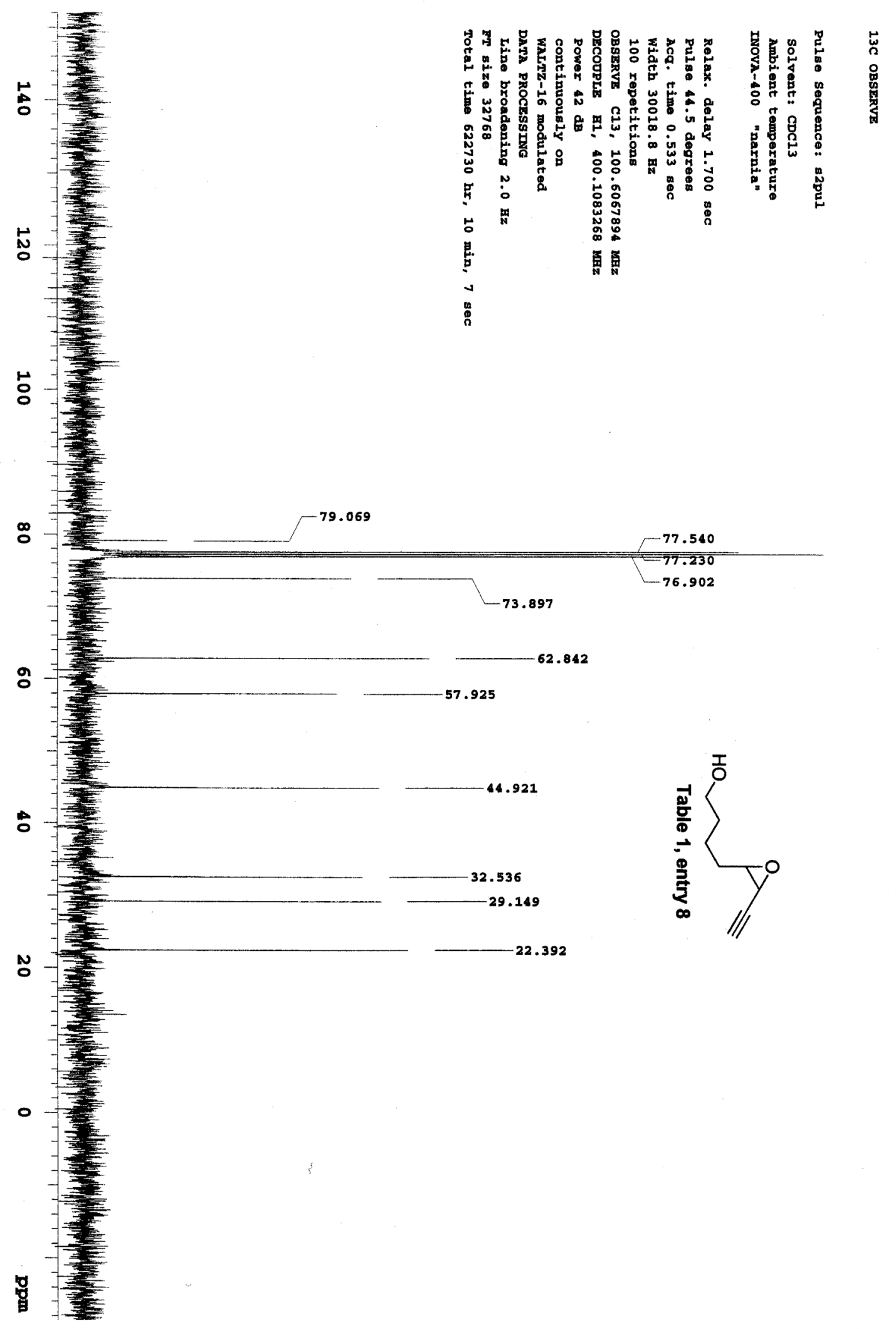




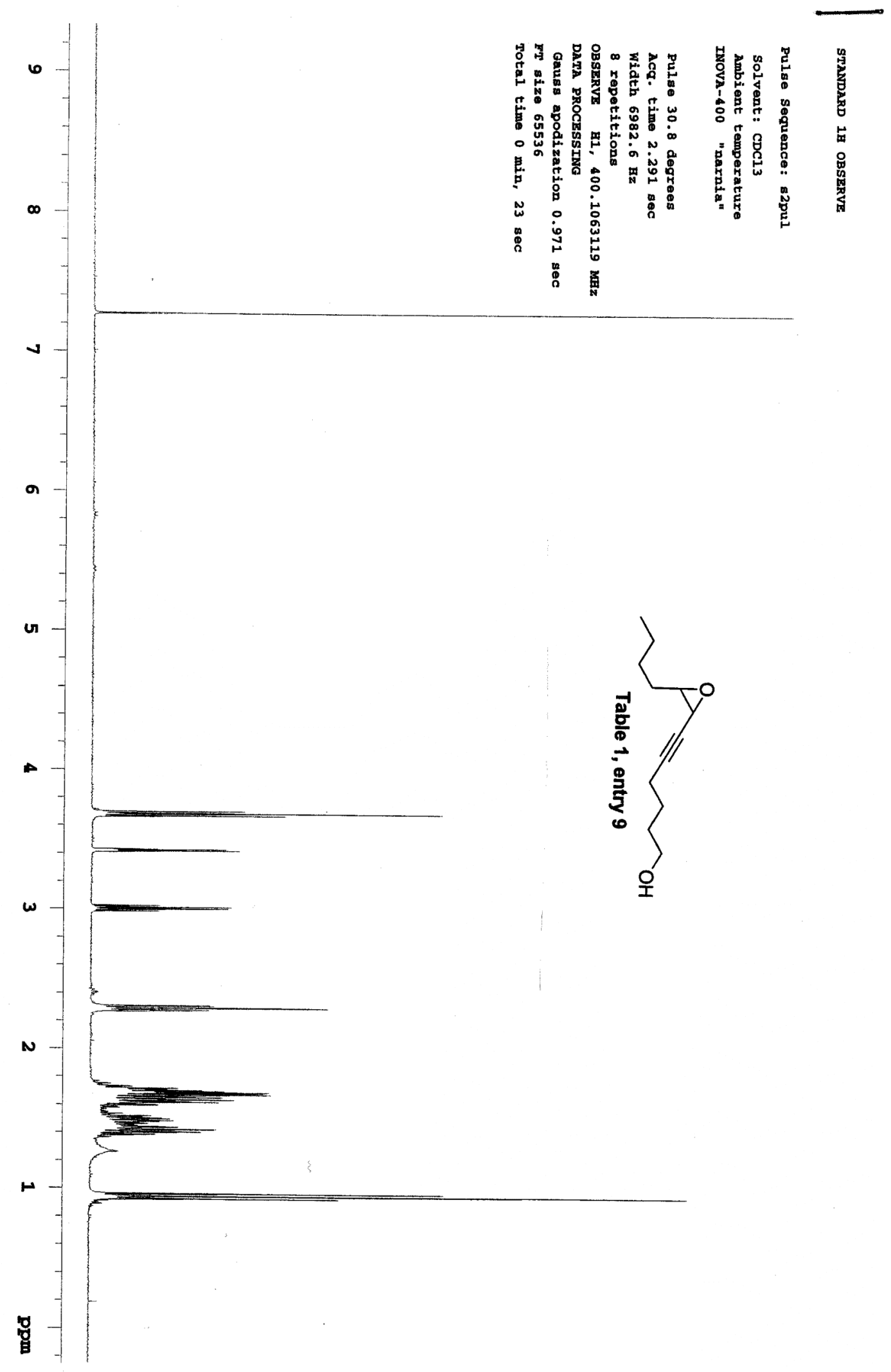




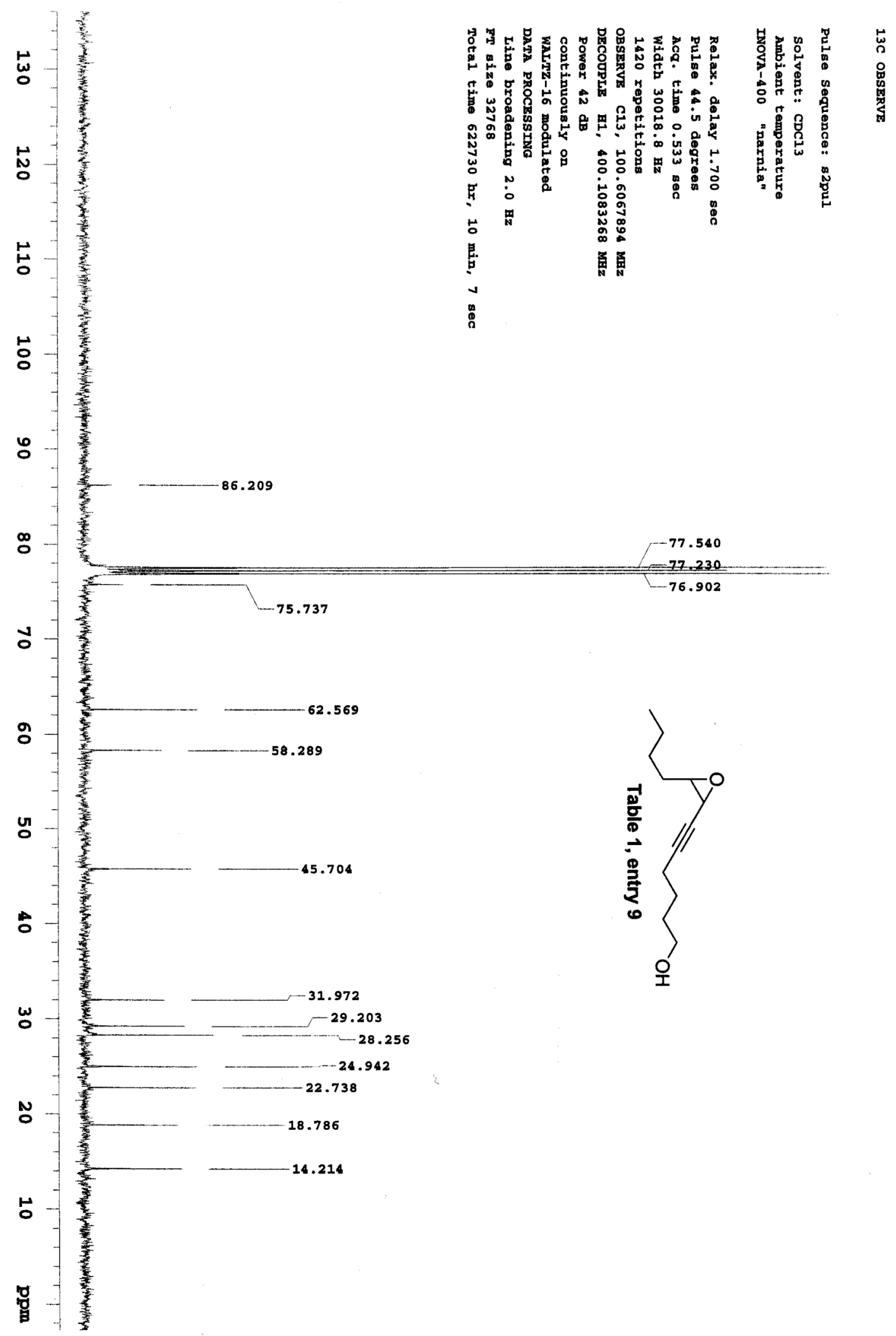



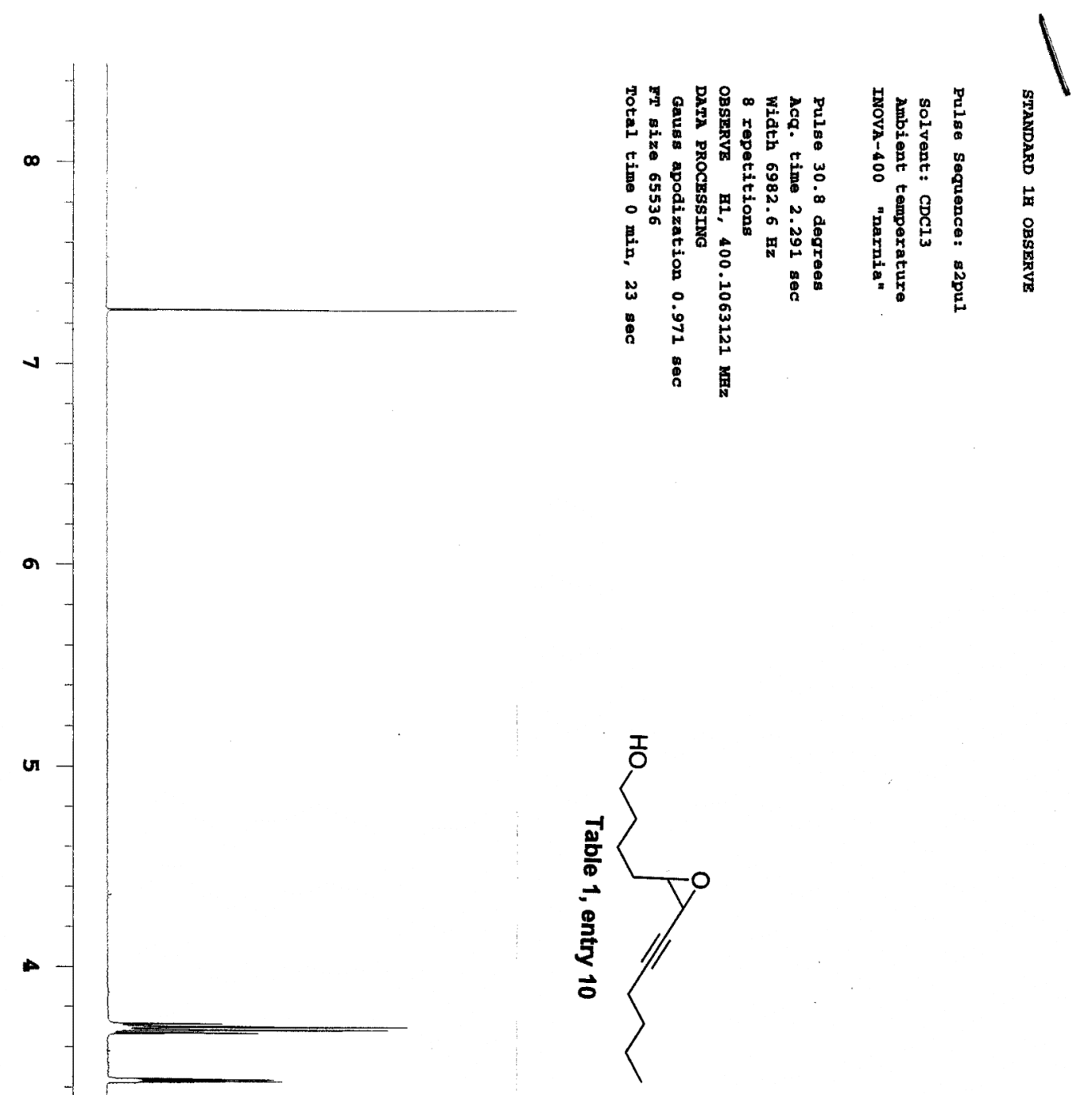

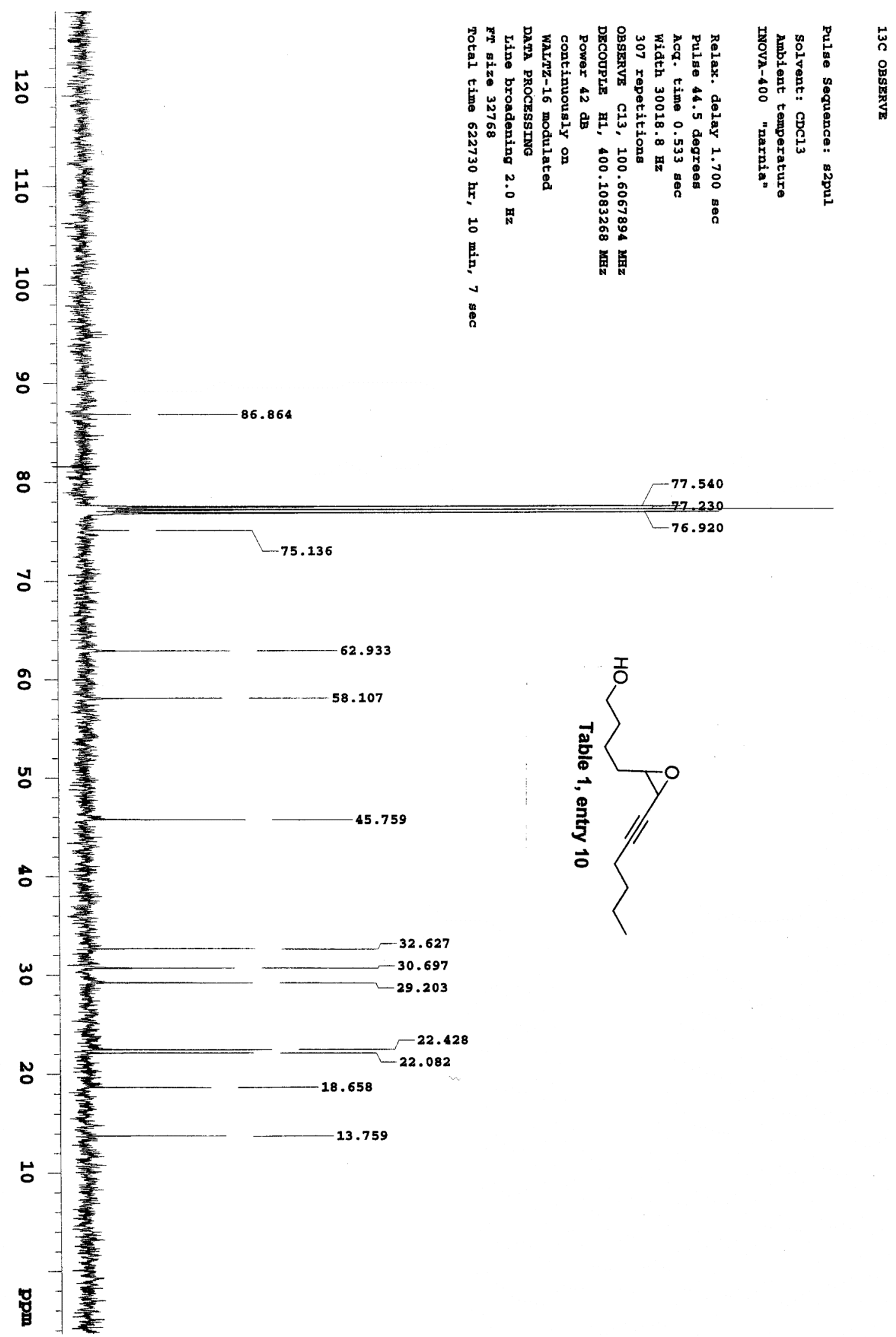


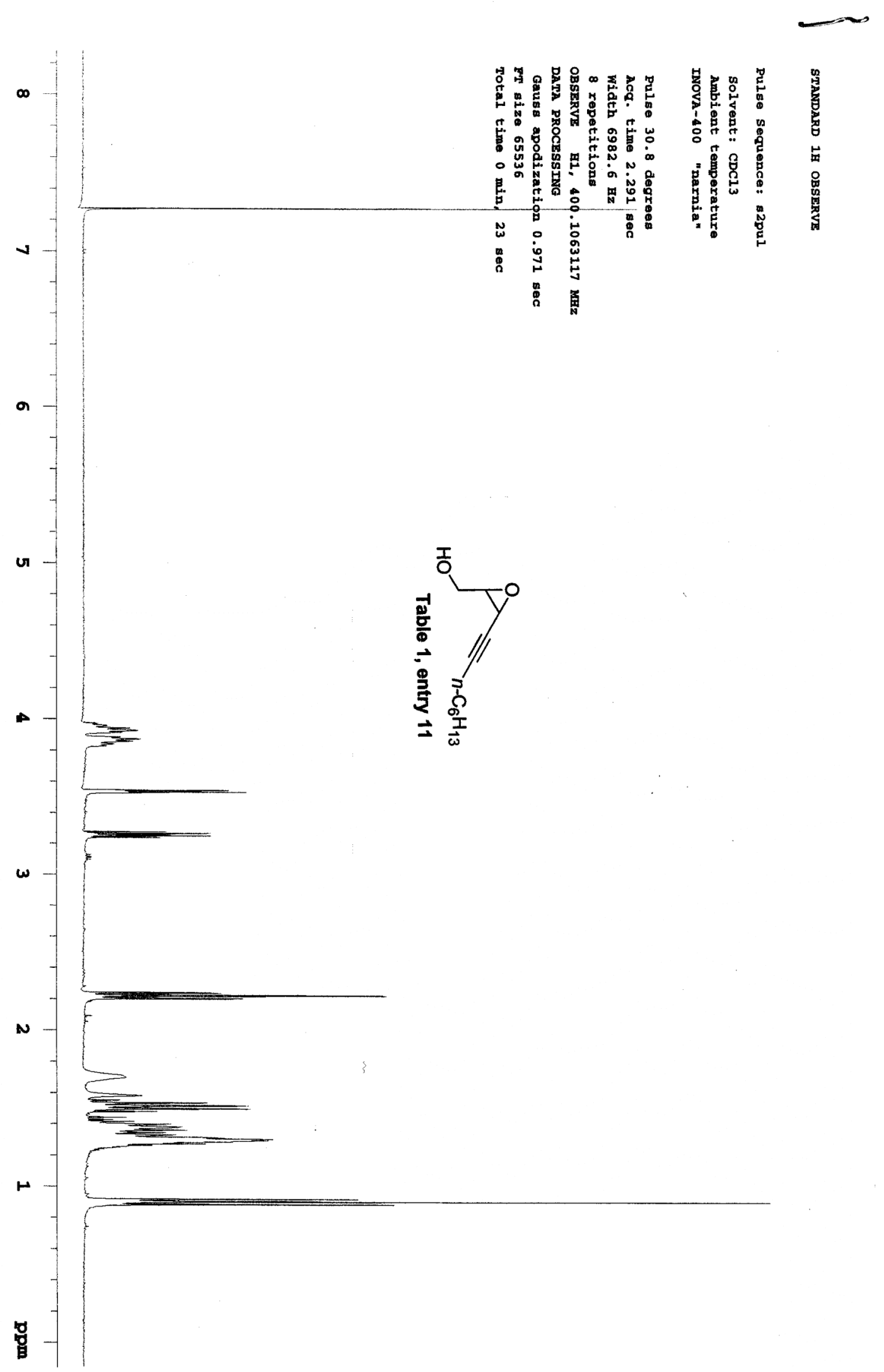




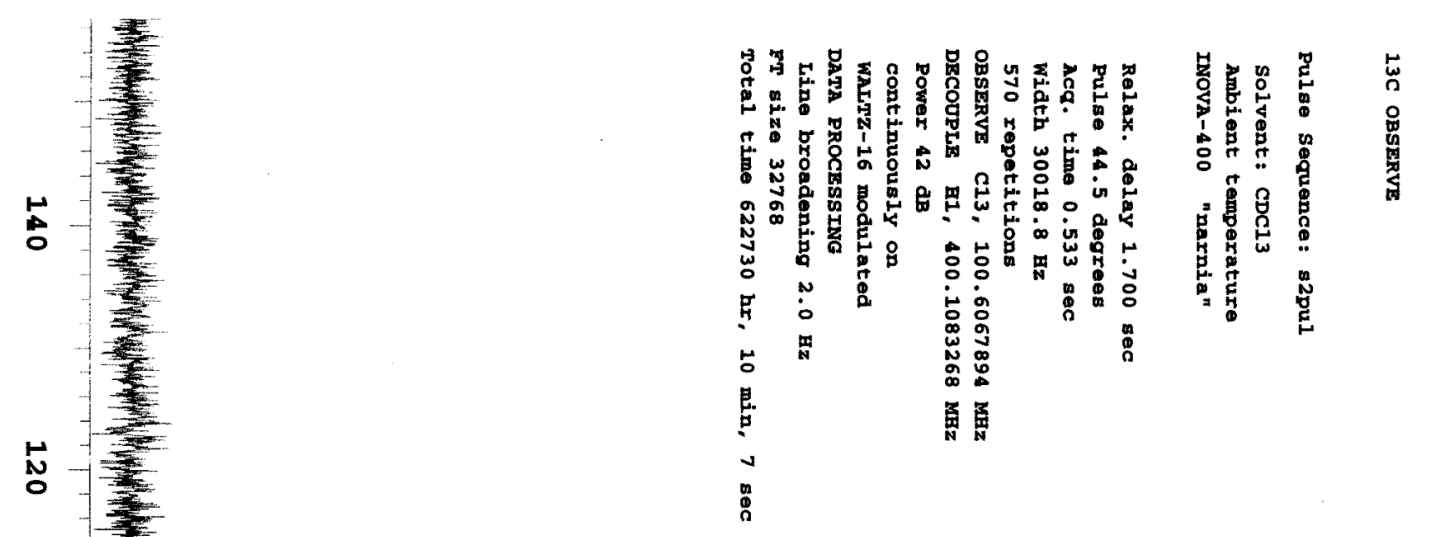

옹

용

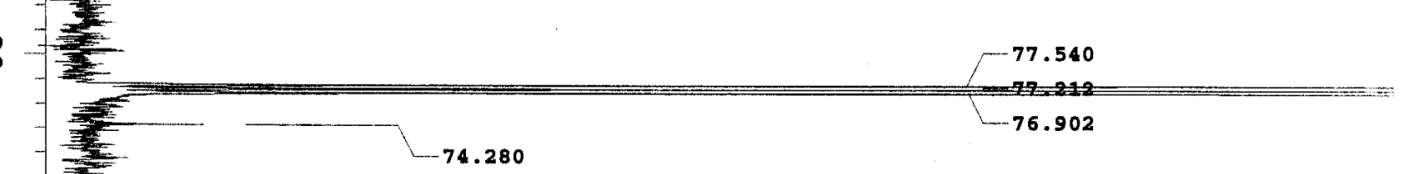

음

응
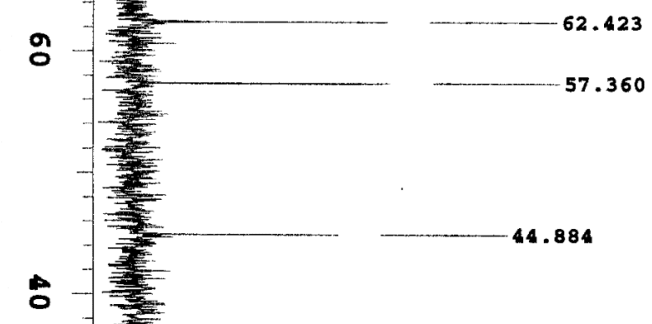

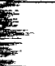

$-57.360$

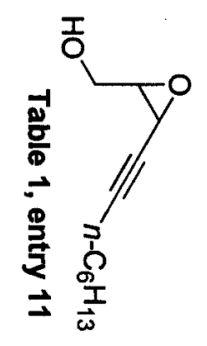

N

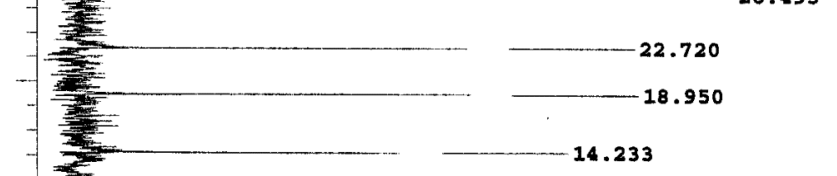

0

胃萧 


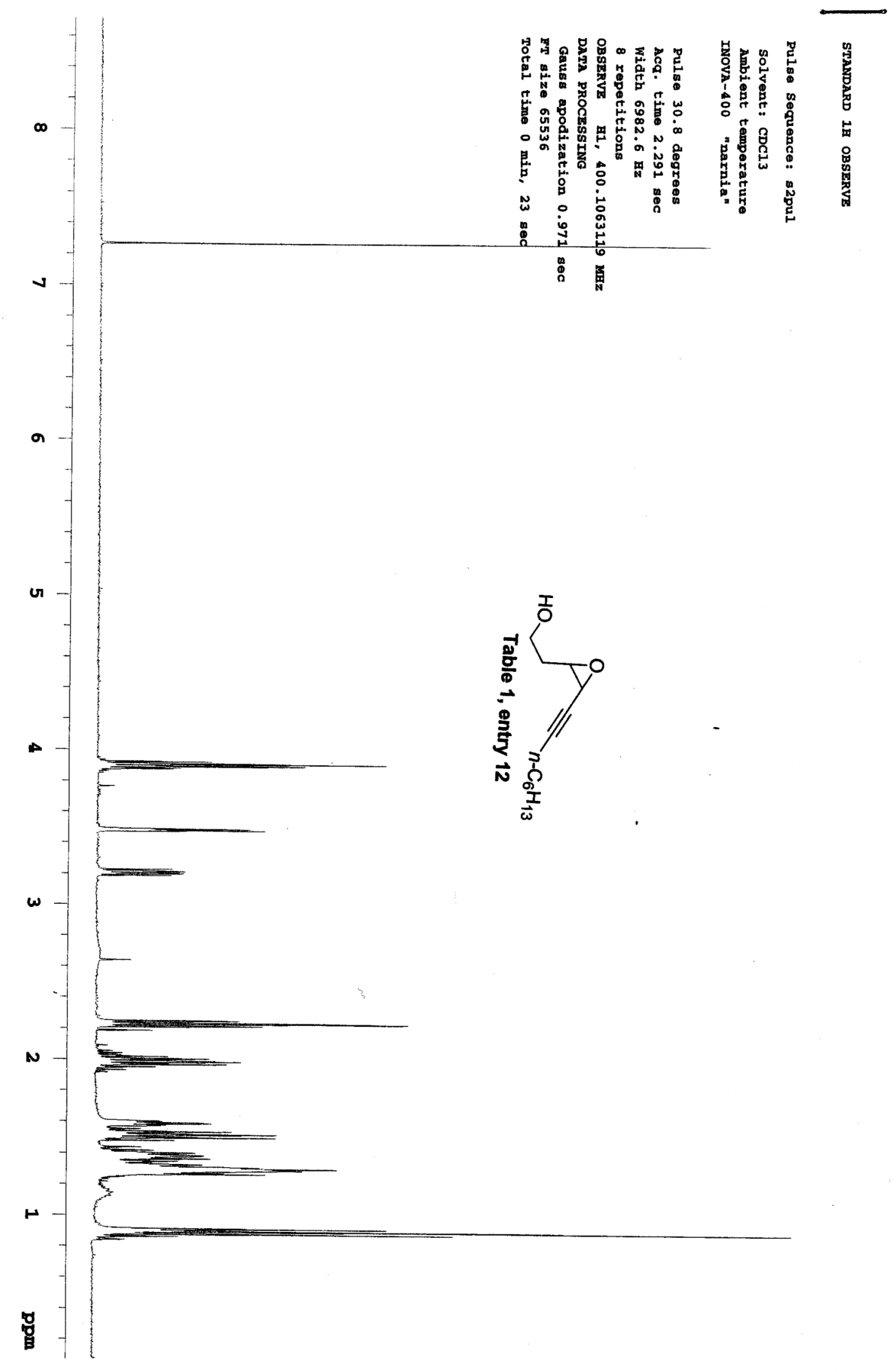




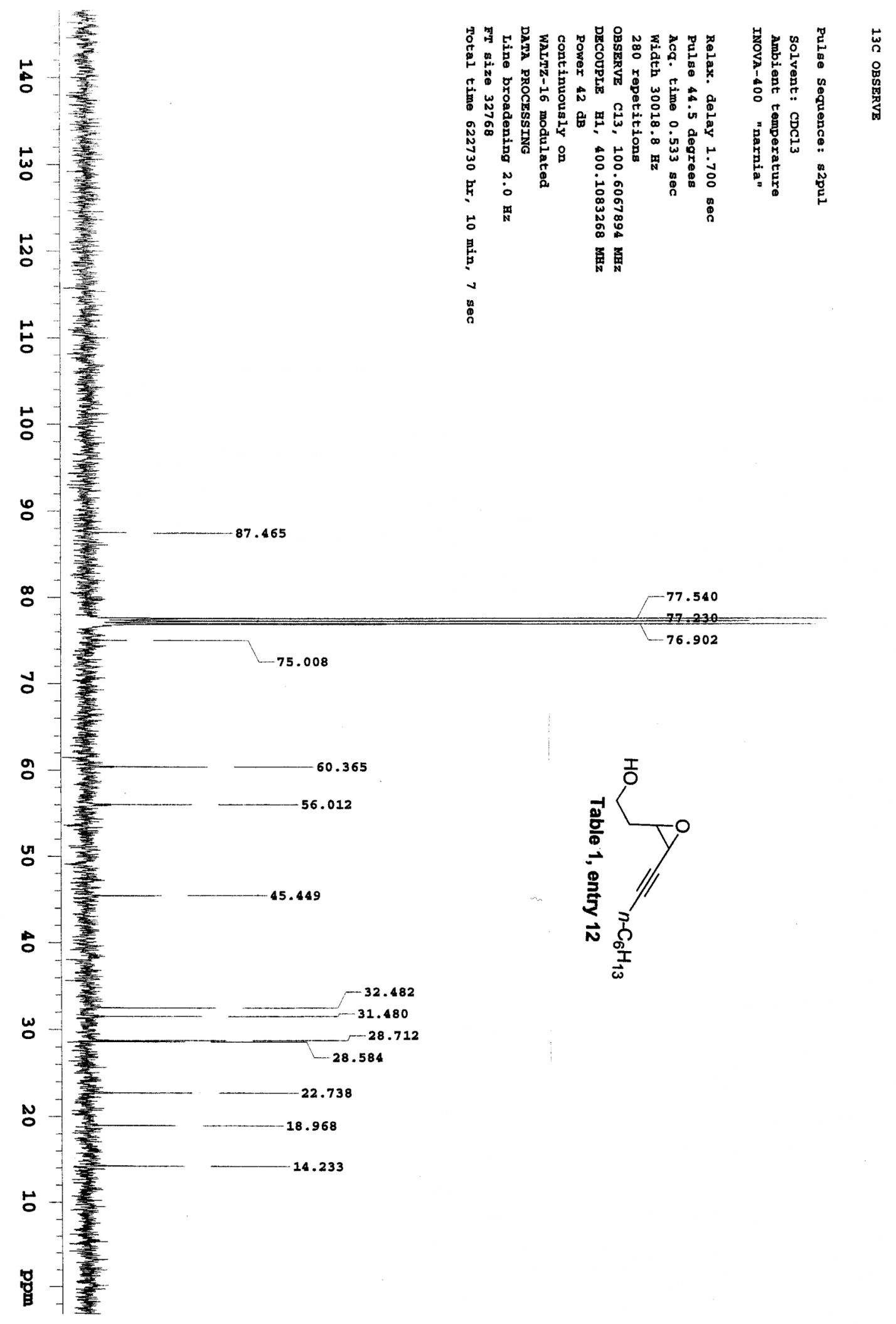




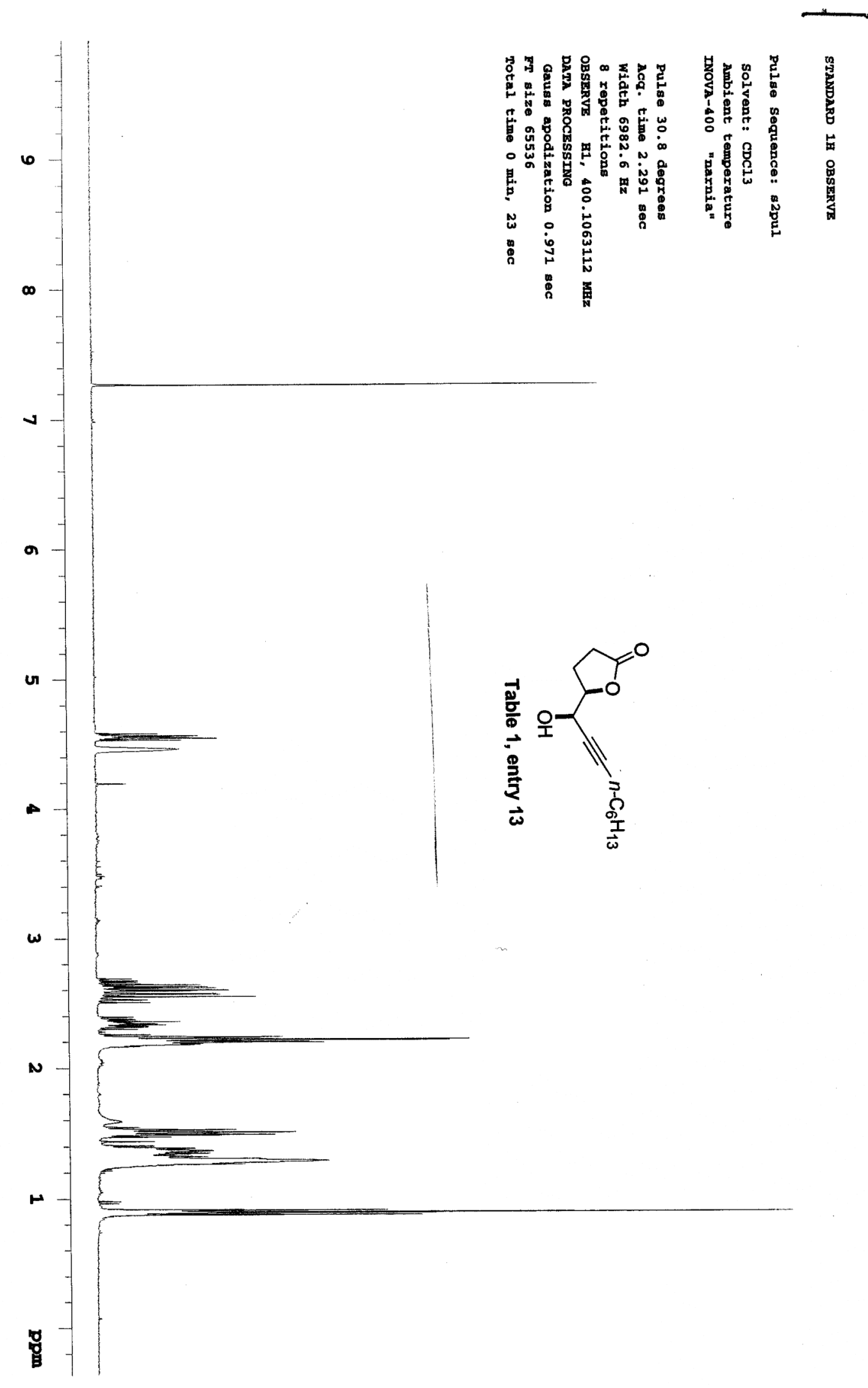




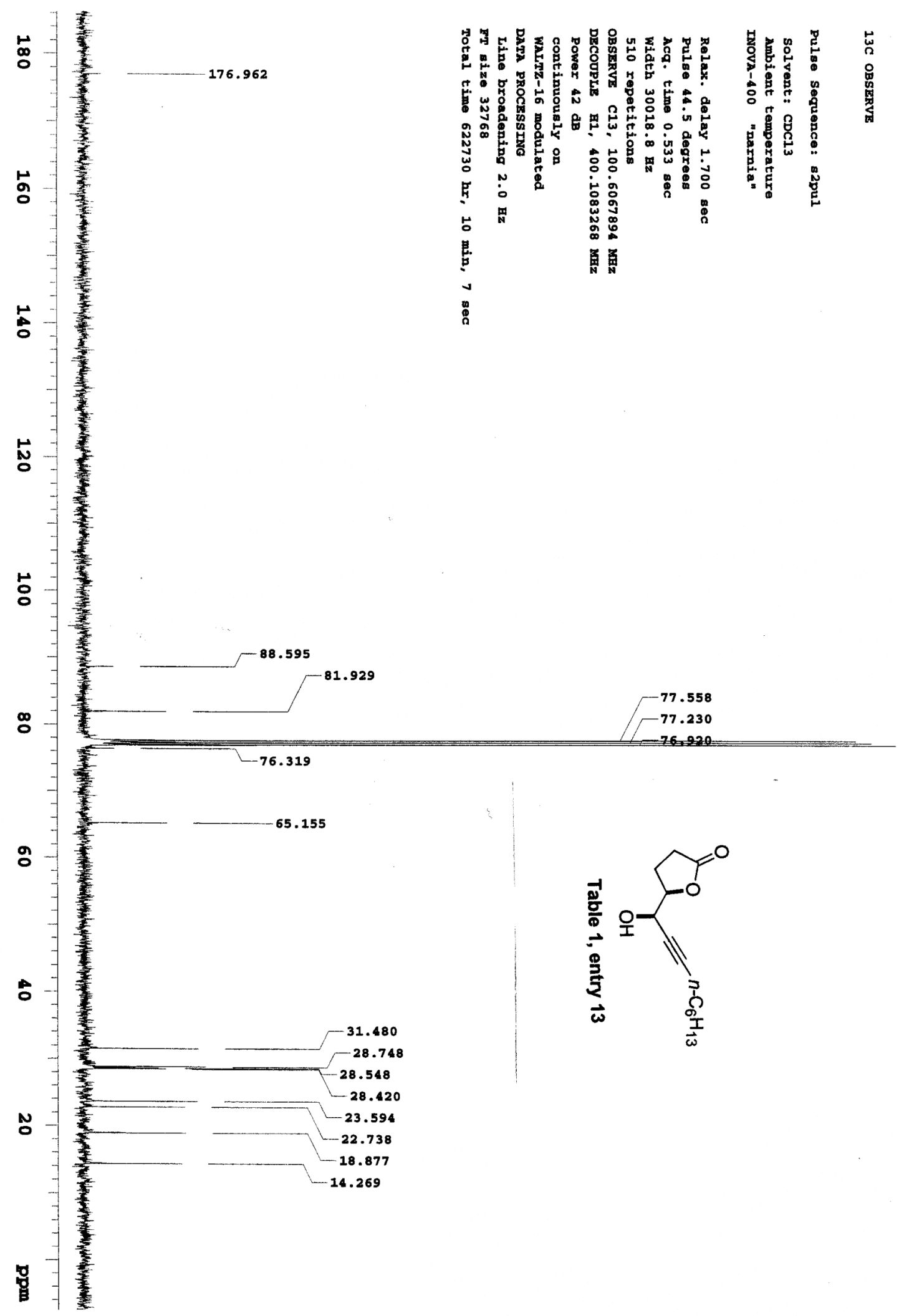




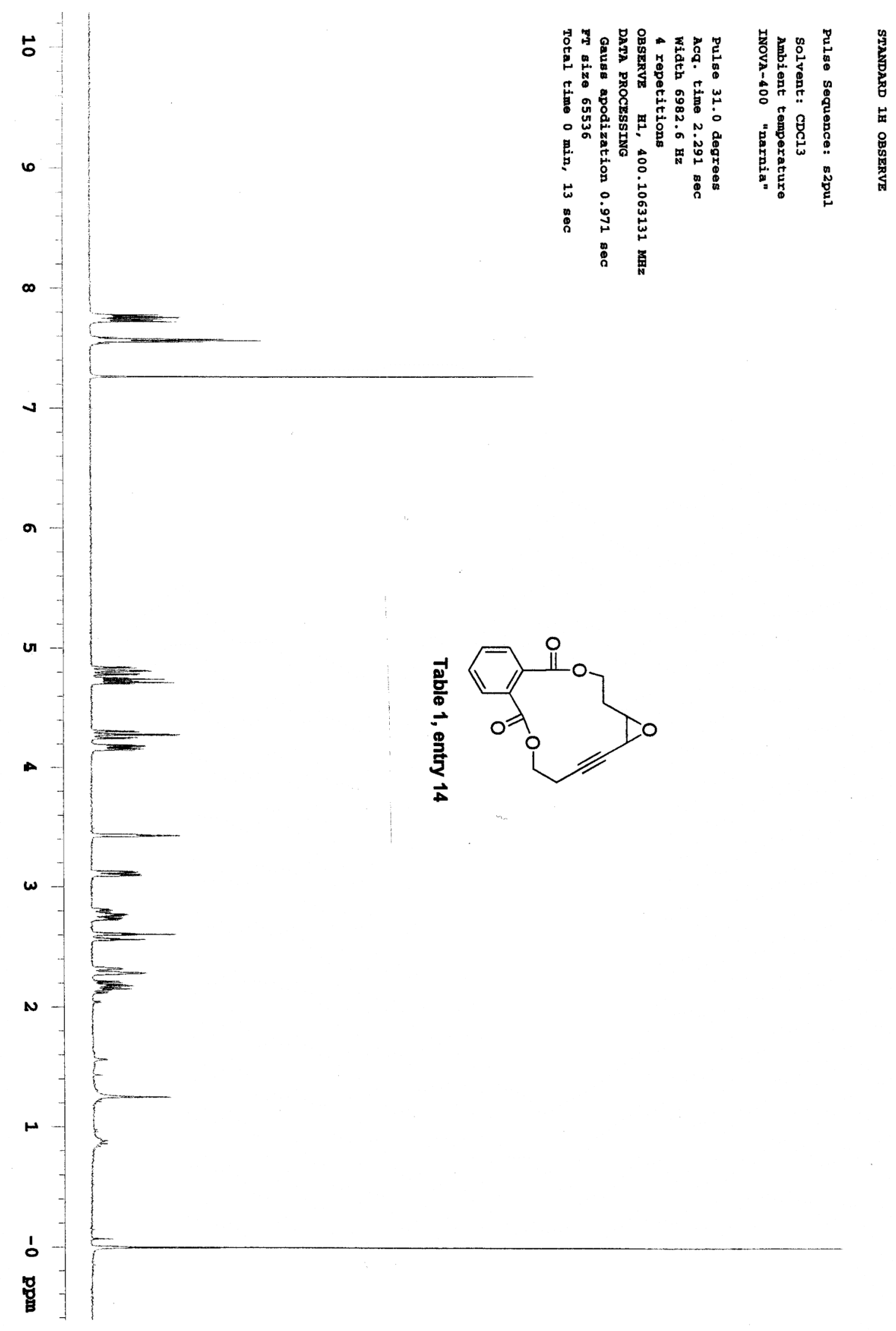



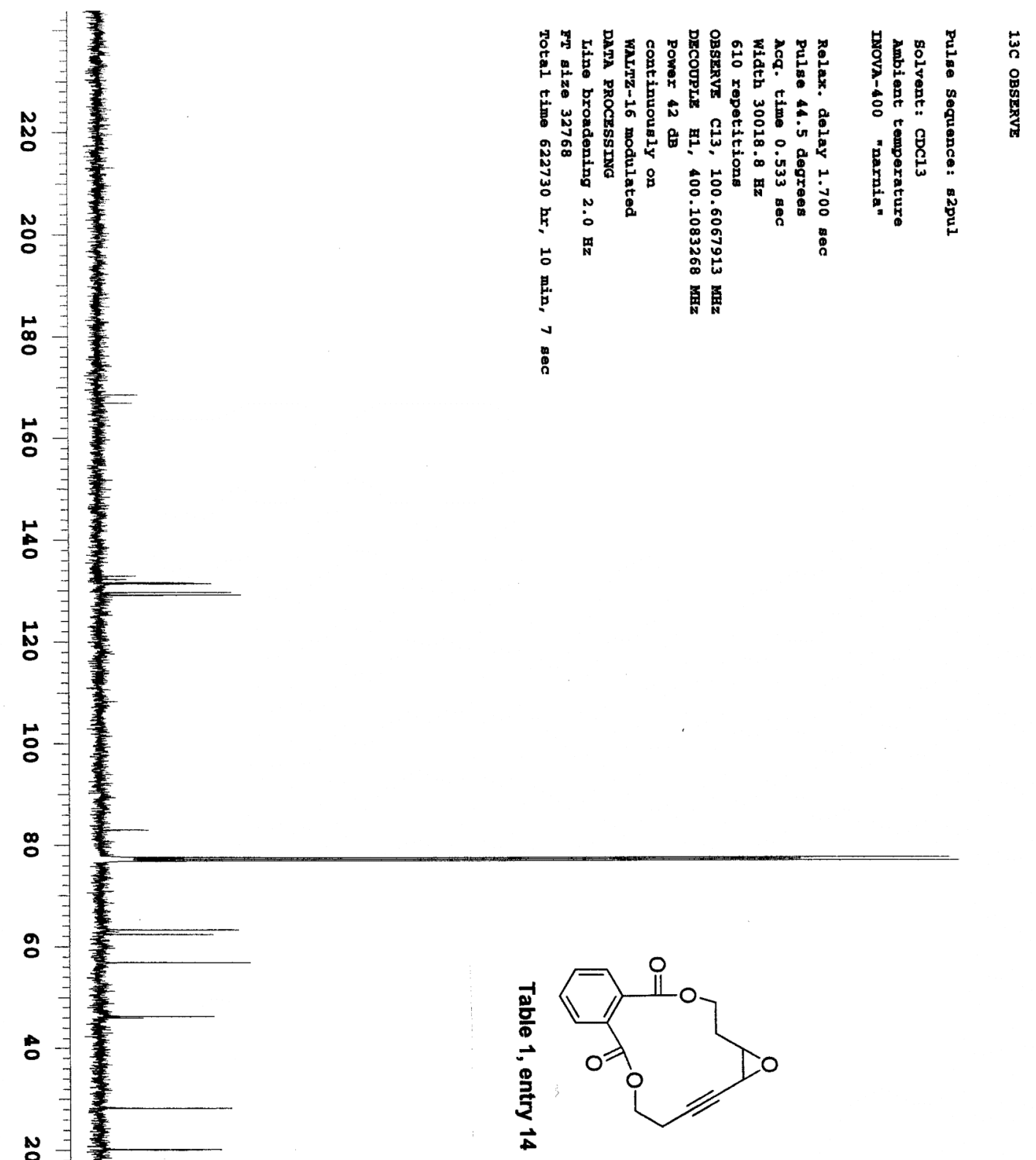


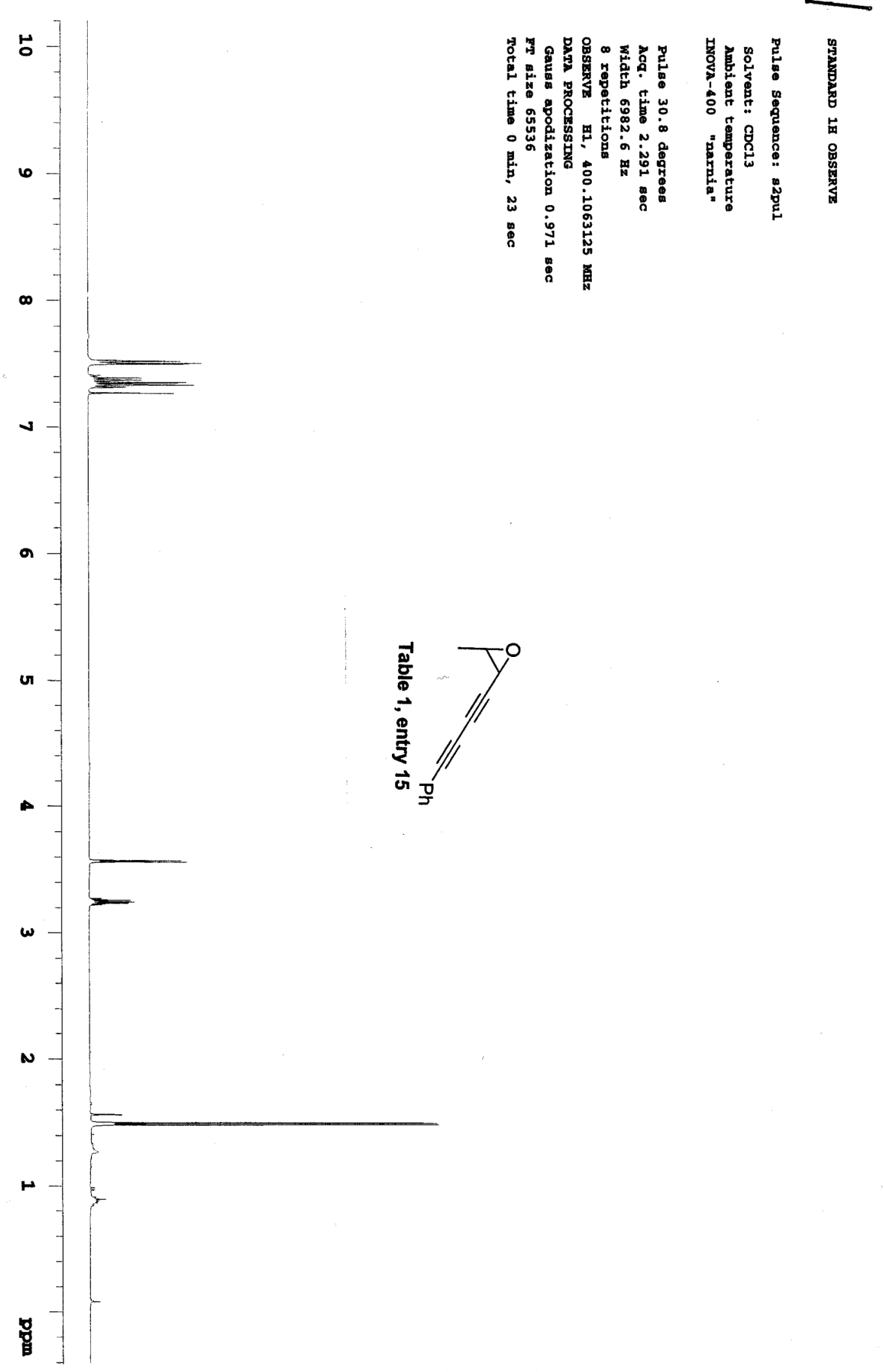




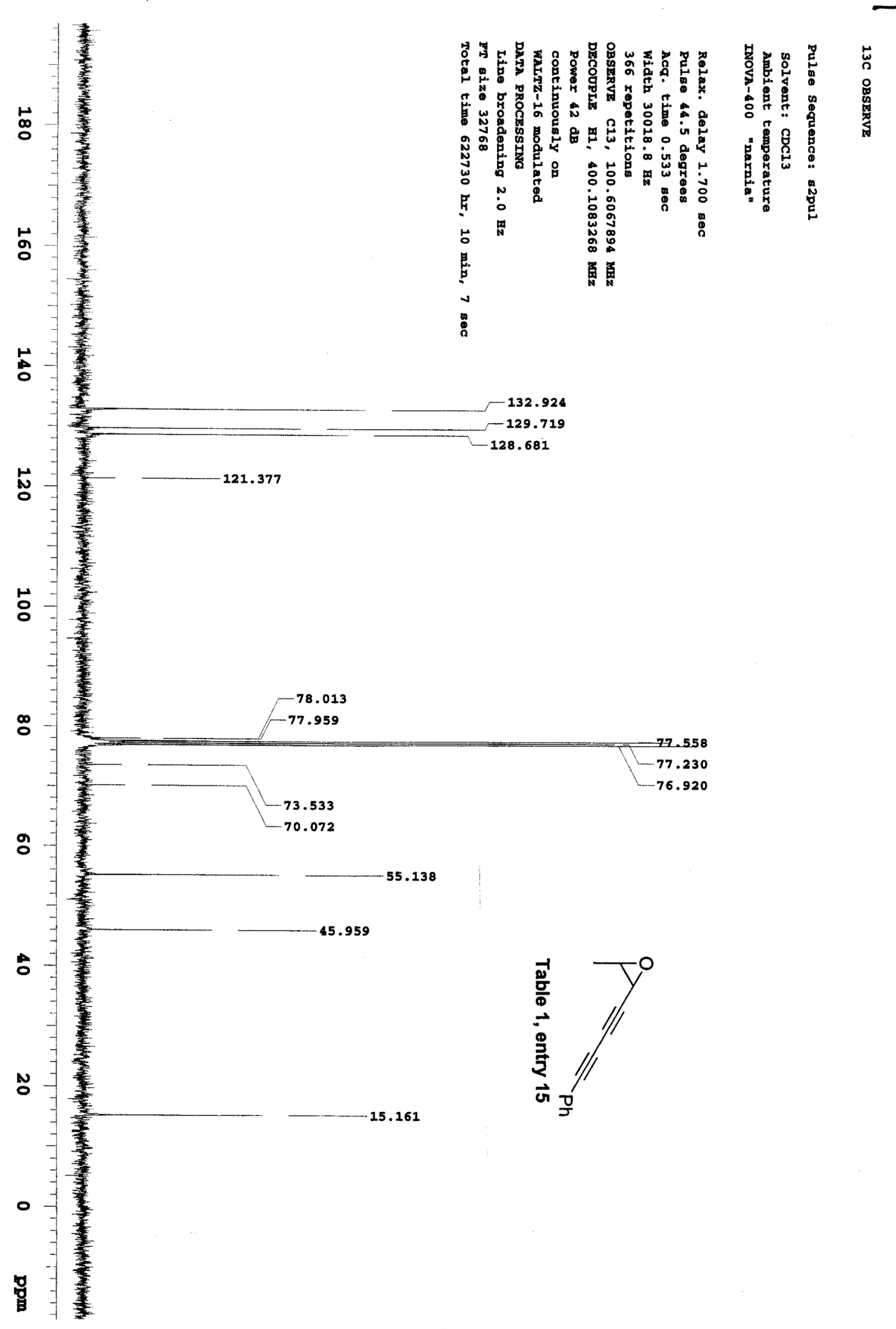

